Aus dem Fachbereich Medizin

der Johann Wolfgang Goethe-Universität

Frankfurt am Main

aus dem

Zentrum der Chirurgie

Klinik für Mund-, Kiefer- und Plastische Gesichtschirurgie

Direktor: Prof. Dr. Dr. Dr. Robert Sader

betreut an der

BGU-Berufsgenossenschaftlichen Unfallklinik Frankfurt am Main

Abteilung für Plastische-, Hand- und Rekonstruktive Chirurgie

\title{
Langzeitergebnisse nach plastisch-chirurgischer Defektdeckung durch neurovaskulären Insel-Lappen bei Fingerkuppenverletzungen
}

\author{
Dissertation \\ zur Erlangung des Doktorgrades der Medizin \\ des Fachbereichs Medizin \\ der Johann Wolfgang Goethe-Universität \\ Frankfurt am Main \\ vorgelegt von \\ Natallia Brui \\ aus Baranowitschi \\ Weißrussland
}

Frankfurt am Main, 2020 
Dekan:

Herr Prof. Dr. Stefan Zeuzem

Referent:

Herr Prof. Dr. Dr. Michael Sauerbier

Korreferent:

Herr Prof. Dr. Johannes Frank

Tag der mündlichen Prüfung: $\quad 18$ März 2021 


\section{Inhaltsverzeichnis}

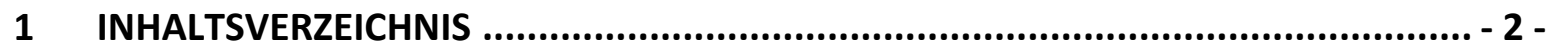

2 ABBILDUNGSVERZEICHNIS .............................................................. - 6 -

3 TABELLENVERZEICHNIS ........................................................................... 7 -

4 ABKÜRZUNGSVERZEICHNIS ............................................................... 9 -

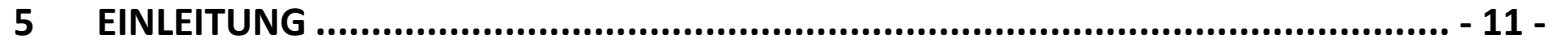

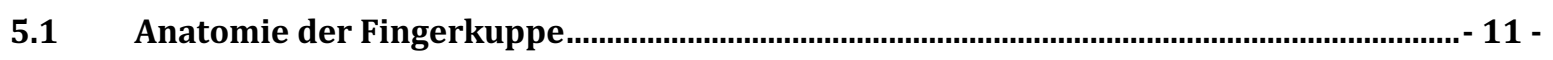

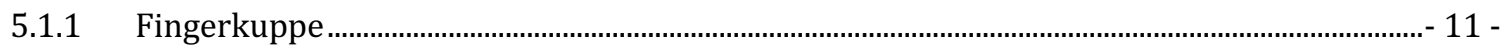

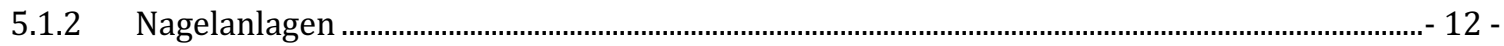

5.1.3 Gefäßversorgung und Innervation der Fingerkuppe...................................................................- 13 -

5.1.4 Pathophysiologie der Fingerkuppe....................................................................................................- 14 -

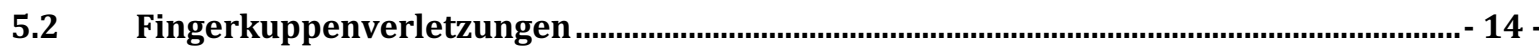

5.3 Präoperative Versorgung von Fingerkuppenverletzungen..............................................- 17 -

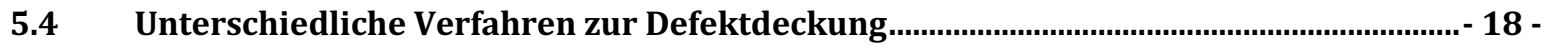

5.4.1 Fingerkuppenamputation ...................................................................................................... 21 -

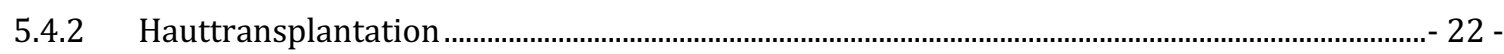

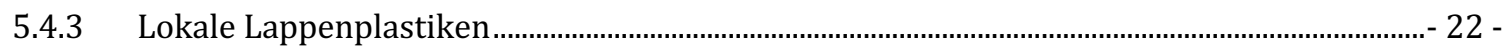

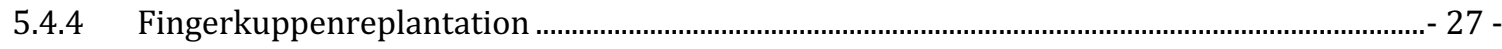

5.4.5 Freie mikrochirurgische Gewebetransplantation ........................................................................- 27 -

5.5 Definition und Entwicklung homodaktylen neurovaskulärer Insel-Lappenplastiken - 28 -

5.6 Vor- und Nachteile neurovaskulärer Insel-Lappenplastiken ............................................. 30 -

5.7 Indikationen und Kontraindikationen neurovaskulärer Insel- Lappenplastiken ......... 31 -

$5.8 \quad$ Verlauf der Operationsvorbereitung ……................................................................. 32 -

Inhaltsverzeichnis - 2 - 
5.8.1 Patientenaufklärung.........................................................................................................- 32 -

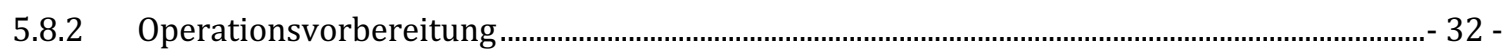

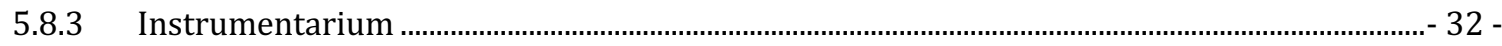

5.8.4 Anästhesie und Lagerung................................................................................................- 33 -

$5.9 \quad$ Unterschiedliche Operationstechniken ….................................................................... 33 -

5.9.1 Chirurgische Anatomie..................................................................................................................- 33 -

5.9.2 Operationstechnik mit anterograden homodigitalen neurovaskulären Insel-Lappen ........- 34 -

5.9.3 Besonderheiten .............................................................................................................................. 36 -

5.9.4 Postoperative Behandlung und Rehabilitation................................................................................. 36 -

5.9.5 Fehler, Gefahren und Komplikationen ...........................................................................................- 36 -

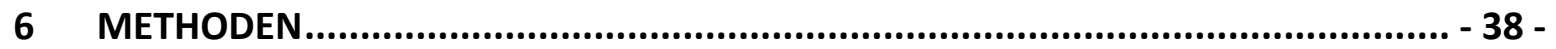

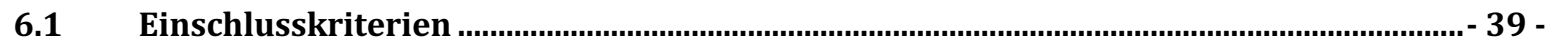

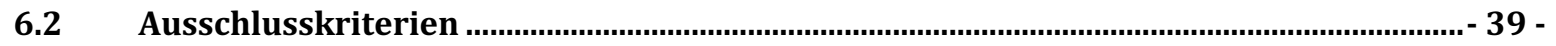

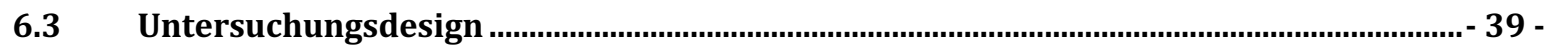

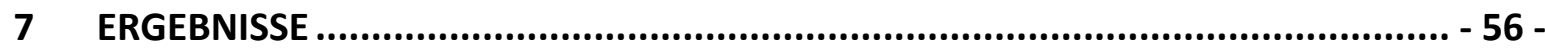

7.1 Auswertung der Patientenakten von 53 Patienten mit 57 Fingerkuppenverletzungen- 56

7.1.1 Alter und Geschlechtsverteilung ...............................................................................................- 56 -

7.1.2 Ort des Unfalls und Art der Fingerkuppenverletzungen ..............................................................- 56 -

7.1.3 Dauer des stationären Aufenthalts ………...............................................................................- 57 -

7.1.4 Lokalisation der Fingerkuppenverletzung …………………………………………………...- 57 -

7.1.5 Hebestelle von neurovaskulären Insel-Lappen...........................................................................- 61 -

7.1.6 Frühkomplikationen und Notwendigkeit von Folgeoperationen...............................................- 62 -

7.2 Patientenbefragung von 28 Patienten mit 29 Fingerkuppenverletzungen ...................- 62 -

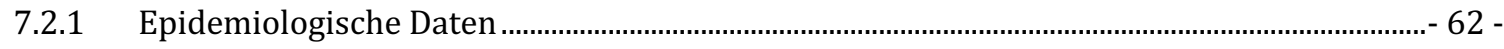

7.2.2 Vorerkrankungen, Medikamente und Raucheranamnese .............................................................- 63 -

7.2.3 Dauer der Nachuntersuchung, Arbeitsunfähigkeit und endgültigen Heilung..........................- 63 -

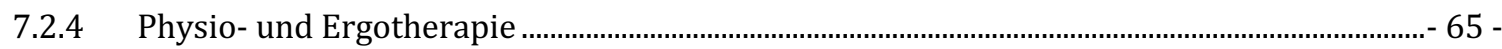

7.2.5 Schmerzempfindlichkeit in Ruhe und unter Belastung ...............................................................- 66 Inhaltsverzeichnis - 3 - 
7.2.6 Subjektive bzw. objektive Bewertung von Schweißbildung und Temperatur .......................- 66 -

7.2.7 Langzeitkomplikationen und Probleme an der Entnahmestelle .................................................- 66 -

7.2.8 Subjektive Patientenzufriedenheit und DASH-Score .........................................................................- 67 -

7.3 Nachuntersuchung von 24 Patienten mit 25 rekonstruierten Fingerkuppen durch 27

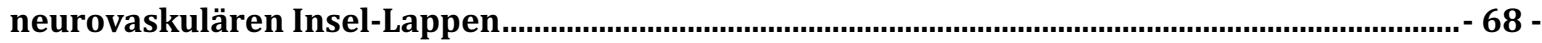

7.3.1 Lappengröße (Länge und Breite) ...................................................................................................- 68 -

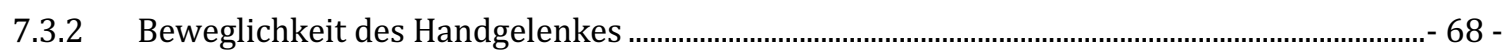

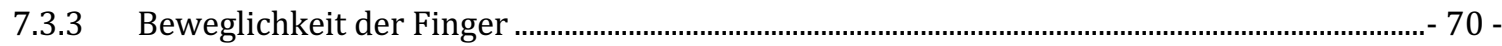

7.3.4 Kraftmessung der Finger.........................................................................................................................- 72 -

7.3.5 Kraftmessung des Handgelenkes mit dem Jamar-Dynamometer Stufe 2 2...............................- 72 -

7.3.6 Zwei-Punkte-Diskrimination (2-PD)..............................................................................................- 73 -

7.3.7 Semmes-Weinstein Monofilament-Test (SWMT) ...........................................................................- 74 -

7.3.8 Narbenbeschreibung und Vancouver Scar Scale (VSS) ................................................................- 75

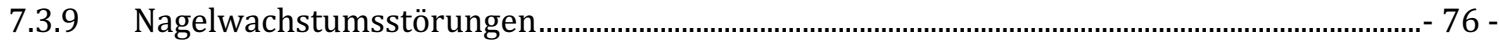

7.4 Fotodokumentation der Finger im Vergleich zur gesunden Seite..................................... 77 -

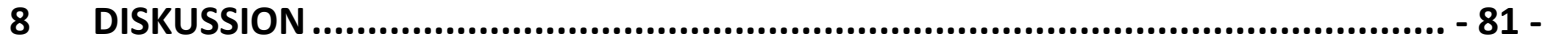

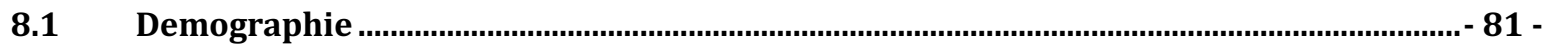

8.2 Lokalisation und Klassifikation der Fingerkuppenverletzungen ........................................ 82 -

8.3 Dauer des stationären Aufenthaltes und der Arbeitsunfähigkeitsdauer ..........................- 83 -

8.4 Patientenzufriedenheit, ästhetisches Outcome und DASH-Score.......................................... 85 -

8.5 Narbenbeschaffenheit, Weichteildeckung und Schmezempfindlichkeit ..........................- 86 -

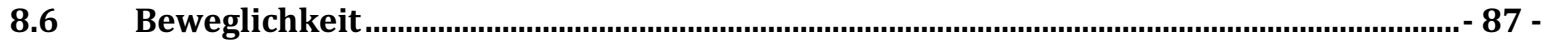

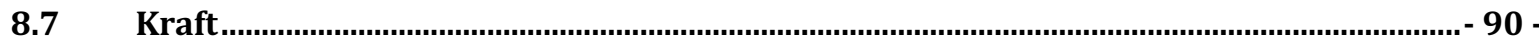

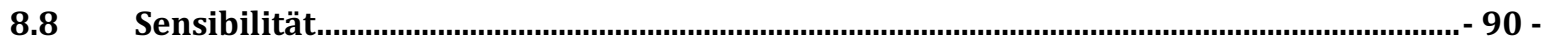

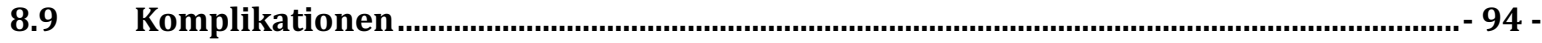

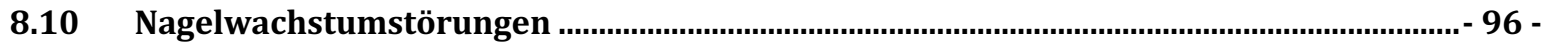

Inhaltsverzeichnis $\quad-4$ - 


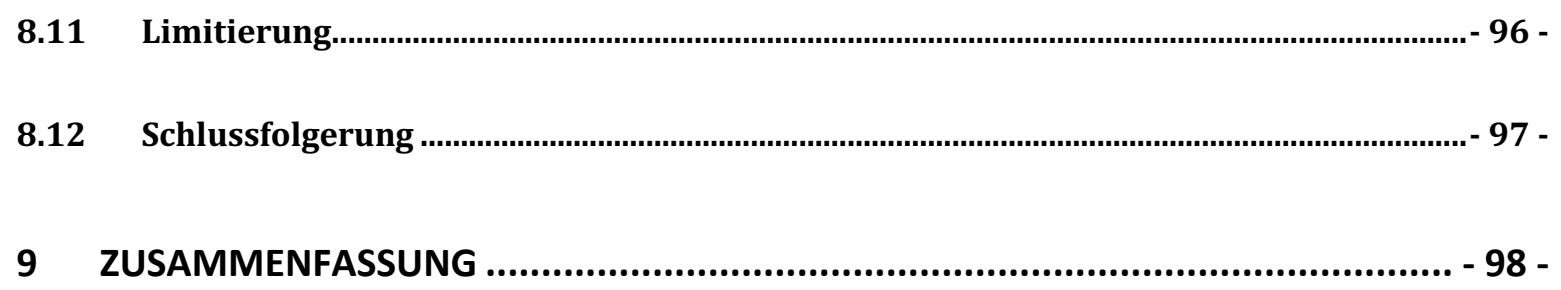

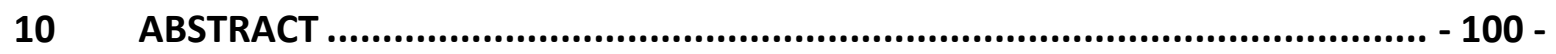

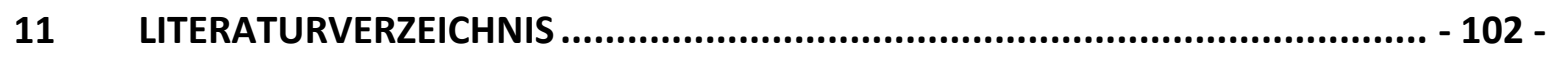

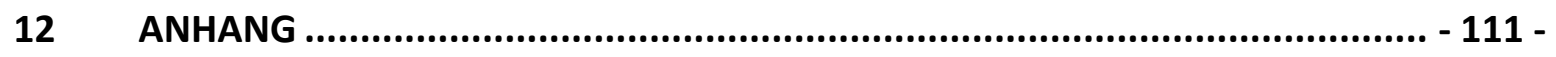

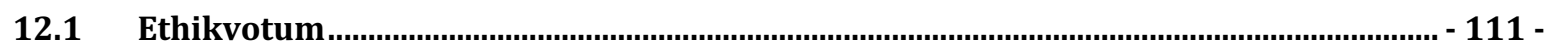

12.2 Patienteninformation und Einwilligungserklärung..................................................... - 113 -

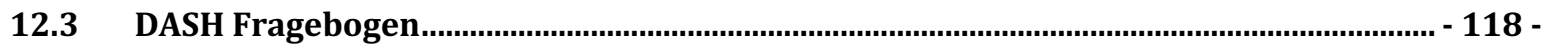

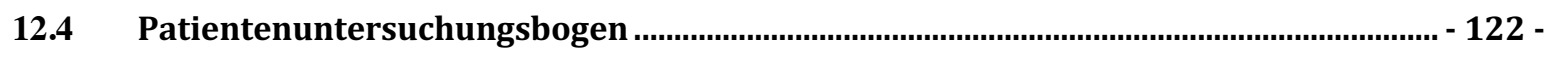

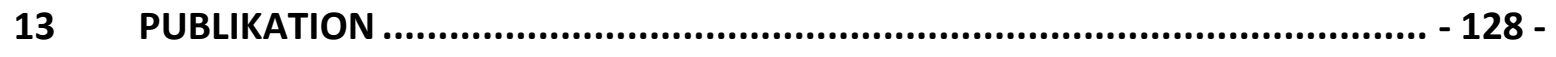

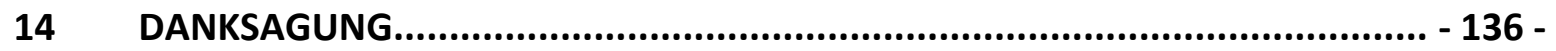

15 SCHRIFTLICHE ERKLÄRUNG ....................................................... 137 -

Inhaltsverzeichnis $\quad-5-$ 


\section{Abbildungsverzeichnis}

Abbildung 1: Anatomie der Fingerkuppe und Nagelanlage (Nachgezeichnet nach G. Kohnle ${ }^{8}$ )................. - 12 -

Abbildung 2: Einteilung der Verletzungszonen nach Allen ${ }^{31}$.............................................................................. - 15 -

Abbildung 3: Einteilung der Verletzungsrichtungen nach Allen ${ }^{31}$...................................................................... - 16 -

Abbildung 4: Rekonstruktive Leiter der plastisch-chirurgischen Defektdeckung nach Majno ${ }^{40}$.................. - 18 -

Abbildung 5: Rekonstruktiver Aufzug der plastisch-chirurgischen Defektdeckung nach Gottlieb und

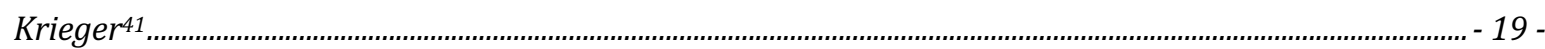

Abbildung 6: Operationsschritte der palmaren Dehnungslappenplastik nach Moberg ${ }^{82}$............................... - 24 -

Abbildung 7: Cross-Finger-Lappenplastik.............................................................................................................. - 26 -

Abbildung 8: Operationsschritte bei anterograden homodigitalen neurovaskulären Insel-Lappen .......... - 35 -

Abbildung 9: Numerische Rating-Skala ................................................................................................................... - 41

Abbildung 10: Messung der Extension und Flexion des Handgelenks................................................................. - 43 -

Abbildung 11: Messung der Radialduktion und Ulnarduktion des Handgelenks ............................................ - 44 -

Abbildung 12: Messung der Supination und Pronation des Handgelenks ......................................................... - 45 -

Abbildung 13: Flexion im Grundgelenk des Fingers................................................................................................ 46 -

Abbildung 14: Flexion im Mittel- und Endgelenk des Fingers .................................................................................. - 46 -

Abbildung 15: Flexion im Grund- und Endgelenk des Daumens........................................................................... - 47 -

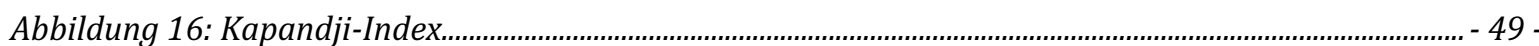

Abbildung 17: Kraftmessung zwischen Daumen und Zeigerfinger mittels „Jamar®“" Pinch-Gauge........... - 50 -

Abbildung 18: Kraftmessung der Handmuskulatur mittels „Jamar ${ }^{\circledR}$ “ Dynamometer Stufe 2 ..................... - 51 -

Abbildung 19: Zwei-Punkte-Diskrimination in mm nach Weber...................................................................... - 52 -

Abbildung 20: Sensibilität an der Fingerkuppe mittels Semmes-Weinstein Monofilament-Test................. - 53 -

Abbildung 21: Lim-Klassifikation des Krallennagels..........................................................................................- 54 -

Abbildung 22: Anzahl der in die Studie aufgenommenen Patienten der verschiedenen Altersgruppen... - 56 -

Abbildung 23: Häufigkeit aller verletzten Fingerkuppen ..................................................................................... - 58 -

Abbildung 24: Häufigkeit der verletzten Fingerkuppen, welche durch neurovaskuläre Insel-Lappenplastik

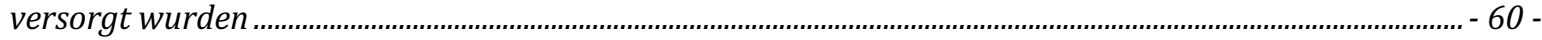

Abbildung 25: Häufigkeit der ausgewählten Hebestelle der neurovaskulären Insel-Lappen ....................... - 61 -

Abbildung 26: Anzahl nachuntersuchte Patienten in den verschiedenen Altersgruppen............................... - 63 -

Abbildung 27: Auswertung der Zwei-Punkte-Diskrimination ……………............................................................... - 74 -

Abbildung 28: Auswertung des Semmes-Weinstein Monofilament-Testes......................................................... - 75 -

Abbildung 29: Auswertung der Vancouver Scar Scale........................................................................................... - 76 -

Abbildung 30: Pre-, intra- und postoperative Bilder einer Fingerkuppenverletzung am Zeigerfinger ..... - 78 -

Abbildung 31: Röntgen-, pre-, intra- und postoperative Bilder einer Fingerkuppenverletzung am Mittelfinger. $-80$
Abbildungsverzeichnis
$-6-$ 


\section{Tabellenverzeichnis}

Tabelle 1: Klassifikation der Verletzungszonen nach Allen ${ }^{31}$...............................................................................- 15 -

Tabelle 2: Klassifikation der Verletzungsrichtungen nach Allen ${ }^{31}$...................................................................... - 16 -

Tabelle 3: Vor- und Nachteile neurovaskulärer Insel-Lappenplastiken ............................................................. - 30 -

Tabelle 4: Indikationen und Kontraindikationen neurovaskulärer Insel-Lappenplastiken ......................... - 31 -

Tabelle 5: Strickland und Glogovac Kriterien.................................................................................................. - 48 -

Tabelle 6: Anzahl der verletzten und durch neurovaskuläre Insel-Lappen versorgten Fingerkuppen...... - 59 -

Tabelle 7: Häufigkeit der ausgewählten Hebestelle der neurovaskulären Insel-Lappen ................................ - 61 -

Tabelle 8: Dauer der Arbeitsunfähigkeit beim Arbeitsunfall und häuslichem Unfall....................................... - 64 -

Tabelle 9: Endgültige Heilungsdauer beim Arbeitsunfall und häuslichem Unfall............................................ - 65 -

Tabelle 10: Anzahl der Physio- und Ergotherapie.................................................................................................... - 65 -

Tabelle 11: Werte der Schmerzempfindlichkeit in Ruhe und unter Belastung anhand der numerischen

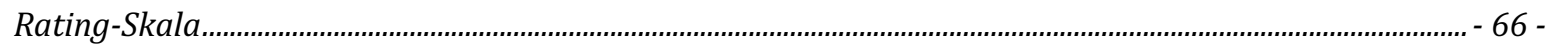

Tabelle 12: Der Mittelwert des DASH-Score für alle befragten Patienten.............................................................. - 67 -

Tabelle 13: Der Mittelwert des DASH-Score für Patienten ohne Störfaktoren .................................................. - 67 -

Tabelle 14: Mittelwerte DASH-Score zwischen allen Patienten und Patienten ohne Störfaktoren............. - 68 -

Tabelle 15: Mittelwerte der aktiven Handbeweglichkeit für die jeweils gesunde und betroffene Hand... - 69 -

Tabelle 16: Unterschied der aktiven Beweglichkeit des gesunden und betroffenen Handgelenkes............ - 70 -

Tabelle 17: Die mittlere Werte der Flexion im Grund-, proximalen- und distalen Interphalangealgelenk sowie

TAM der gesunden und betroffenen Finger ................................................................................................................ - 71 -

Tabelle 18: Unterschied der Flexion im Grund-, proximalen- und distalen Interphalangealgelenk sowie TAM der gesunden und betroffenen Finger ..................................................................................................................... - 71 -

Tabelle 19: Mittlere Werte bei der Messung der Fingerkraft am gesunden und betroffenen Finger mittels

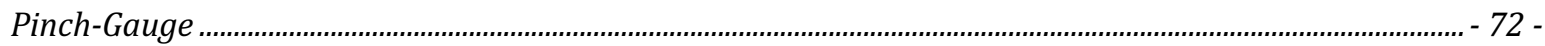

Tabelle 20: Mittlere Werte der Kraftmessung mittels Jamar-Dynamometer der gesunden und betroffenen

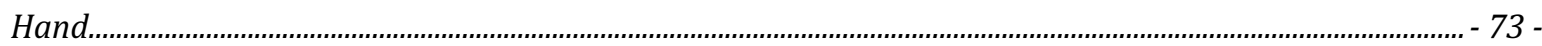

Tabelle 21: Werte des Semmes-Weinstein Monofilament-Testes der neurovaskulären Insel-Lappen und Fingerkuppe der Gegenseite.................................................................................................................................... 75 -

Tabelle 22: Ergebnisse der Behandlungsdauer von Fingerkuppenverletzungen mit einem Semiokklusionsverband................................................................................................................................ - 84 -

Tabelle 23: Ergebnisse der Patientenzufriedenheit nach der Rekonstruktion von Fingerkuppenverletzungen mittels neurovaskulärer Insel-Lappenplastik........................................................................................................ - 85 -

Tabelle 24: Ergebnisse des Streckdefizits im PIP- und DIP-Gelenk nach der Rekonstruktion von Fingerkuppenverletzungen mittels neurovaskulärer Insel-Lappenplastik ........................................................ - 88 -

Tabellenverzeichnis - 7 - 
Tabelle 25: Vergleich der 2-PD nach der Rekonstruktion der Fingerkuppenverletzungen mittels Semiokklusionstherapie und Ergebnissen der eigenen Studie ............................................................................ - 93 -

Tabelle 26: Langzeitkomplikationen andere Studien und unserer ....................................................................... - 95 - 


\section{Abkürzungsverzeichnis}

$\begin{array}{ll}\text { A. } & \text { Arteria } \\ \text { Aa. } & \text { Arteriae } \\ \text { Abb. } & \text { Abbildung } \\ \text { ADL } & \text { Activities of Daily Living (Aktivitäten des täglichen Lebens) } \\ \text { ASHT } & \text { American Society of Hand Therapist } \\ \text { bspw. } & \text { beispielsweise } \\ \text { bzw. } & \text { beziehungsweise } \\ \text { ca. } & \text { circa } \\ \text { D } & \text { Digitus }\end{array}$

DASH Disability of the Arm, Shoulder and Hand (Fragebogen zur Fubktionskapazität von Arm, Schulter und Hand, DASH-Fragebogen)

DIP distale Interphalangealgelenk

etc. et cetera

FDP Flexor digitorum profundus

FDS Flexor digitorum superficialis

GG Grundgelenk

ggf. gegebenenfalls

IP Interphalangealgelenk

m. Musculus

MCP Metakarpophalangealgelenk

MW Mittelwert

n Anzahl

$\mathrm{N} \quad$ Newton (F-Kraft)

N. Nervus

NRS Numerische Rating-Skala

$\mathrm{p} \quad$ Signifikanz

2-PD Zwei-Punkte-Diskrimination

PIP Proximale Interphalangealgelenk

PNB Pulp, Nail and Bone (Pulpa, Nagel und Knochen Klassifikation) Abkürzungsverzeichnis - 9 - 
R Range (Spannweite)

ROM Range of Motion (Bewegungsradius)

Tab. Tabelle

TAM Total Active Motion (Beweglichkeit der Finger)

vs.

versus (gegen)

VSS Vancouver Scar Scale

SD Standard Deviation (Standardabweichung)

SWMT Semmes-Weinstein Monofilament-Test

z.B. zum Beispiel

Abkürzungsverzeichnis $\quad-10$ - 


\section{Einleitung}

\subsection{Anatomie der Fingerkuppe}

Verletzungen der Fingerkuppen stellen einen häufigen Grund für die Vorstellung in der

Notaufnahme dar. ${ }^{1-3}$ Kenntnisse der Anatomie (Abb. 1) und Physiologie der Finger und Fingerkuppe sind eine wichtige Voraussetzung für eine zeitnahe und suffiziente handchirurgische Therapie bei Fingerkuppenverletzungen.

\subsubsection{Fingerkuppe}

Die Fingerkuppe wird als distal zur Ansatzstelle der Sehne des M. extensor digitorum und Sehne des M. flexor digitorum profundus liegenden Fingerendphalanx definiert. ${ }^{4,5}$ Sie besteht aus Haut, Pulpaskelett, Knochenskelett sowie Nagelanlagen. ${ }^{6,7}$

Die Haut an der palmaren Seite der Fingerkuppe hat ein charakteristisches Leistenmuster (Dermatoglyphen), welches leicht erkennbar und bei jedem Menschen individuell ist. Die Leisten vergrößern die Hautoberfläche und die in der Haut mündenden Schweißdrüsen verbessern die Greiffähigkeit von Gegenständen. ${ }^{8}$

Darunterliegende subkutane Gewebe sind von der Dermis bis zum Periost der distalen Phalanx durch radiär verlaufende bindegewebige Septen gegliedert. ${ }^{9}$ Diese stabilisieren zusammen mit der Nagelplatte die Fingerkuppe beim Druck von außen. ${ }^{8,10}$ Der proximale Anteil der Pulpa enthält anstatt fibröser Septen, sphärisch geformte Lobuli, welche hier die notwendige Flexibilität gewährleisten. ${ }^{11,12}$

Das knöcherne Skelett der Fingerkuppe wird als distale Fingerphalanx bezeichnet. Der Durchmesser der Endphalanx ist an der Basis am breitesten und an der palmaren Seite konkav geformt. Auf der palmaren Fingerspitze setzt die Sehne des M. flexor digitorum profundus (FDP) an, dieser Muskel verläuft entlang des ganzen Fingers. ${ }^{13}$ Die Sehne des M. flexor digitorum superficialis (FDS) sitzt proximal am Interphalangealgelenk fest. ${ }^{14}$ Ganz distal liegt Tuberositas phalangis distalis, welche für die Verankerung des Bindegewebes verantwortlich ist. ${ }^{8,11}$ Auf der dorsalen Seite an der Basis der Endphalanx sind die Pars terminalis der Dorsalaponeurose und der distale Teil der Sehne des M. extensor digitorum befestigt. ${ }^{15}$

Einleitung 


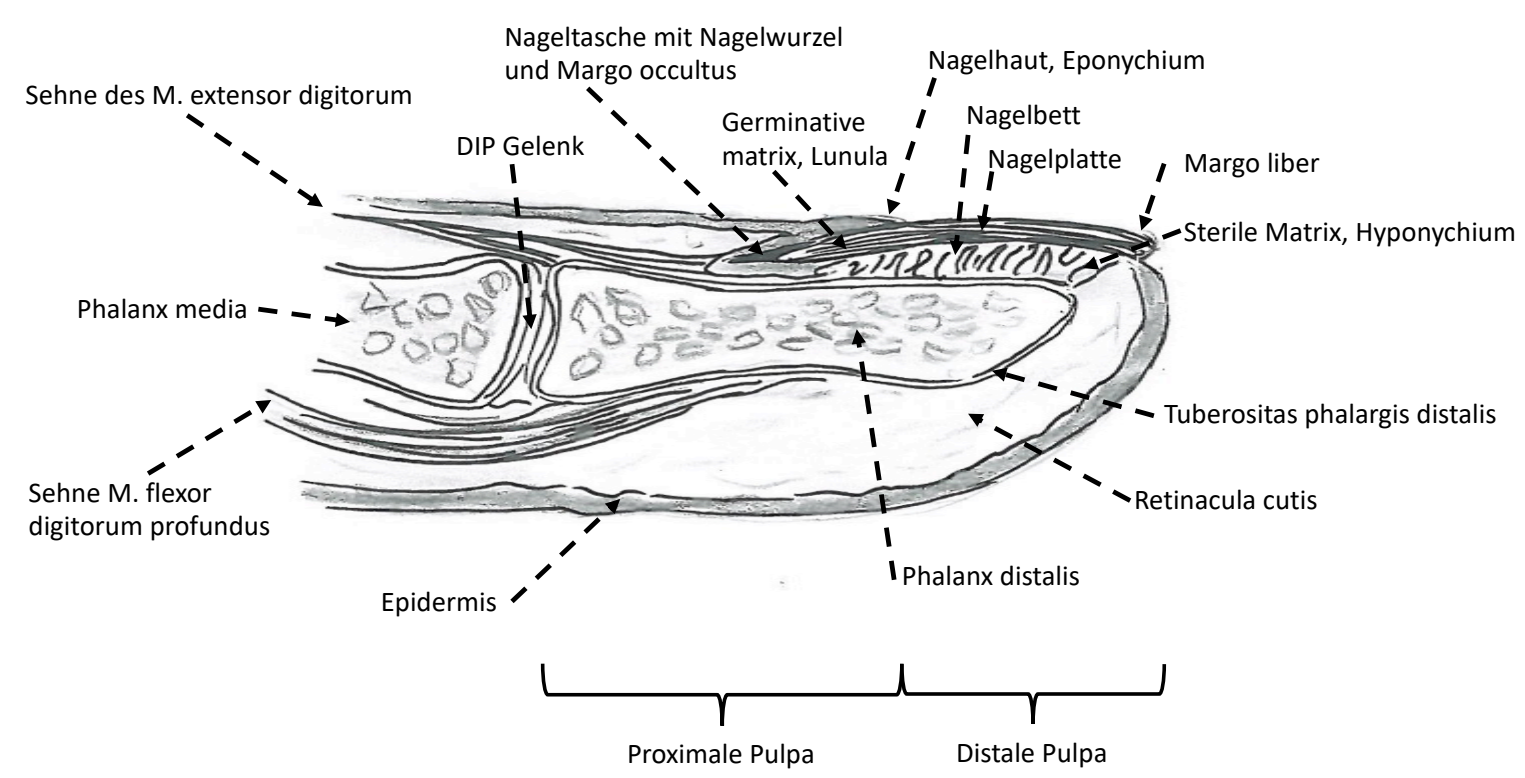

Abbildung 1: Anatomie der Fingerkuppe und Nagelanlage (Nachgezeichnet nach G. Kohnle $^{8}$ )

\subsubsection{Nagelanlagen}

Dorsal an der Endphalanx der Finger und des Daumens befindet sich der konvex geformte Nagel, der als festes Widerlager beim Greifvorgang dient und beispielweise das Kratzen ermöglicht. ${ }^{16}$

Die Nagelplatte besteht aus verhornten Keratinozyten und ist ca. 0,5 mm dick. ${ }^{17}$ Das Nagelwachstum beträgt ca. $0,1 \mathrm{~mm}$ pro Tag und wird von Alter, Geschlecht, Jahreszeit sowie der Belastung beeinflusst. ${ }^{18}$ Die Nagelplatte besteht distal aus einem freien Rand (Margo liber) unter dem eine feine epidermale Kante (Sohlenhorn) liegt. Proximal auf der Nagelplatte befindet sich die Nagelhaut (Eponychium), die die Nageltasche vor dem Eintritt von Fremdkörpern schützt. Unter dem Eponychium liegt die sichtbare germinale Matrix (Lunula) in der Form eines Kreissegments, welche zu 90\% für das Wachstum der Nagelplatte verantwortlich ist. Die sterile Matrix (Hyponychium) oberhalb der Lunula hält die Nagelplatte fest und ist für die restlichen $10 \%$ der Nagelbildung verantwortlich. ${ }^{10}$

Einleitung 
Der proximale Rand der Nagelplatte (Margo occultus) liegt zusammen mit der Nagelwurzel in der 4 bis $6 \mathrm{~mm}$ tiefen Nageltasche. ${ }^{8}$

\subsubsection{Gefäßversorgung und Innervation der Fingerkuppe}

Die Fingerkuppe wird durch die Endäste der beiden Aa. digitales palmaris propriae, welche aus der A. digitales palmaris communis entspringen, mit arteriellem Blut versorgt. Auf der Höhe des Endgelenks im Bereich der distalen Insertion der Sehne des Flexor digitorum profundus (FDP) vereinen sich die palmaren Fingerarterien und bilden mehrere Arkaden zwischen der radialen und palmaren Seite. ${ }^{19}$ Die Arkaden können unterschiedlich geformt sein und nehmen nicht immer die klassische anatomische Stellung ein. ${ }^{20}$ Von diesen palmaren Arkaden verlaufen mehrere Arteriolen zum Fingerende und bilden das Rete arteriosum. Die Arteriolen umrunden die Fingerspitze und vereinen sich mit den Ästen der dorsalen Arkaden von Aa. digitales dorsales. An der dorsalen Seite der Fingerkuppe befinden sich die subunguale arterielle Arkade (Arcus arteriosus dorsalis distalis) als Versorgungsgefäß des Nagelbettes und die oberflächliche arterielle Arkade (Arcus arteriosus dorsalis proximalis) zur Ernährung der Nagelmatrix. ${ }^{21}$

Die venöse Versorgung der Fingerkuppe erfolgt durch die Venae palmares superficiales und die Venae communicantes obliques, welche in die Venae metacarpales dorsales münden. ${ }^{22}$ Dorsale Venen haben oft kleinere Kaliber als palmare und bilden einen Arcus venosus. 23,24 Im Bereich des Fingergrundgelenks überkreuzen sich die Nerven mit Arterien. Die zuvor dorsal liegenden Nerven der Mittelhand befinden sich jetzt am Finger palmar.,25

Die Teilung der beiden Nervi digitales palmares proprii erfolgt häufig auf Höhe der palmaren Beugefalte des DIP-Gelenks. Die terminalen Äste versorgen die Fingerseitenflächen sowie die Fingerkuppe und der dorsale Ast innerviert das Nagelbett. ${ }^{26}$ Die Zahl der terminalen Äste kann am Daumen von mindestens zwei bis sieben und am Finger von zwei bis fünf variieren, ohne wesentlichen Unterschied zwischen der ulnaren und radialen Seite. ${ }^{27}$

Einleitung 


\subsubsection{Pathophysiologie der Fingerkuppe}

Die komplexe Funktionalität der Fingerkuppe als Tastsinnesorgan wird durch die zahlreichen im subkutanem Gewebe verteilten Mechanorezeptoren, Tastkörperchen und Nervenendigungen gewährleistet. ${ }^{8}$

Die Merkel-Testzellen sowie die Ruffini-Körperchen erfassen statische und phasische Teile des Reizes. Meissner-Körperchen in der oberflächlichen Dermis sind für die Druckempfindlichkeit bei der Berührung verantwortlich. ${ }^{28}$ Vater-Pacini-Körperchen und Golgi-Mazzoni-Lamellenkörperchen (Kälterezeptoren) nehmen Beginn oder Ende einer Druckänderung sowie Beschleunigung bzw. Vibration wahr. ${ }^{20,29}$

Zusammenfassend kann die Fingerspitze als Organ der epikritischen Oberflächensensibilität gesehen werden, welche eine wichtige Rolle in der Gegenstandserkennung bzw. Stereognosis, Temperaturempfindlichkeit, Kontaktaufnahme und Schmerzempfindlichkeit spielt.

\subsection{Fingerkuppenverletzungen}

Verletzungen der Fingerkuppen stellen einen häufigen Grund für die Vorstellung in der Notaufnahme dar. ${ }^{1,3,7}$

Abhängig vom Ausmaß der Fingerkuppenverletzungen können Haut, Pulpa, knöchernes Skelett sowie Nagelstrukturen distal der Ansatzstelle der Sehnen des M. extensor digitorum und des M. flexor digitorum profundus des Daumens bzw. der Finger betroffen sein. ${ }^{6,7}$ Am Häufigsten treten Lazerationen und Quetschverletzungen auf, Fingerkuppenamputationen dagegen kommen seltener vor. ${ }^{30}$

In der Literatur werden verschiedene Aufteilungen der Fingerkuppenverletzungen dargestellt, darunter die Klassifikationen nach Allen, ${ }^{31}$ Rosenthal, ${ }^{32}$ Tamai, ${ }^{33}$ Foucher, ${ }^{34}$ Ishikawa, ${ }^{35}$ Fassler ${ }^{4}$ Hirase $^{36}$ und die „Pulp, nail and bone“ Klassifikation ${ }^{37}$ (PNB).

Fast alle Klassifikationen orientieren sich an anatomischen Strukturen oder der Geometrie der Verletzung. Jede der Klassifikationen hat Vor- und Nachteile, im Wesentlichen stellen sie jedoch eine praktische Hilfe für eine einheitliche Beschreibung dar und können orientierende Hinweise zur weiteren medizinischen Versorgung geben.

Einleitung 
Detaillierter wird in der Literatur häufig die Klassifikation nach Allen beschrieben, welche auch in dieser Studie angewandt wird.

Nach der Allen-Klassifikation ${ }^{31}$ von 1980 werden Fingerkuppenverletzungen in vier Zonen (Abb. 2 und Tab. 1) unterteilt:

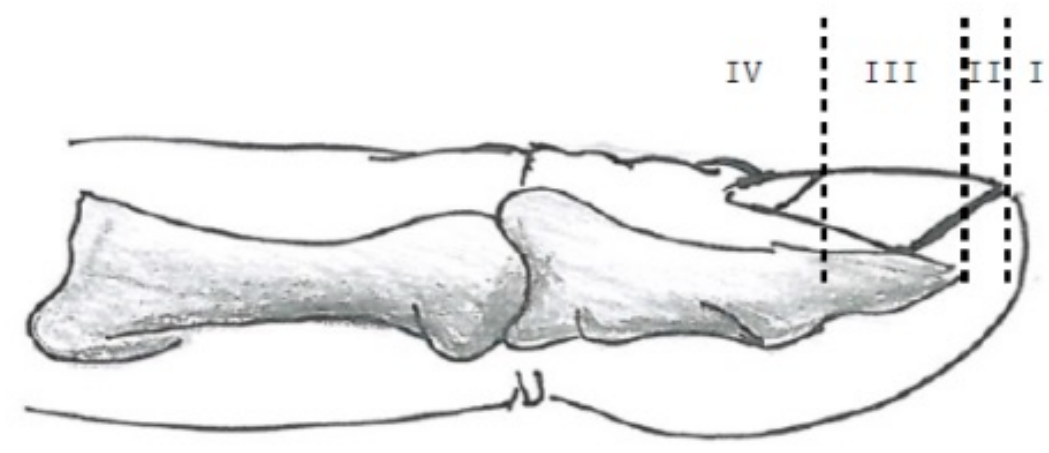

Abbildung 2: Einteilung der Verletzungszonen nach Allen ${ }^{31}$

\begin{tabular}{|l|l|}
\hline Zone I: & $\begin{array}{l}\text { Verletzungen der Fingerkuppe ohne Verletzung des Nagelbettes und ohne } \\
\text { knöcherne Verletzung }\end{array}$ \\
\hline Zone II: & $\begin{array}{l}\text { Verletzungen der Fingerkuppe und des Nagelbettes ohne knöcherne } \\
\text { Verletzung }\end{array}$ \\
\hline Zone III: & $\begin{array}{l}\text { Verletzung der Fingerkuppe mit Teilverletzung der distalen Phalanx distal der } \\
\text { Lunula }\end{array}$ \\
\hline Zone IV: & $\begin{array}{l}\text { Verletzungen der Fingerkuppe mit Verletzung der Lunula und proximal } \\
\text { davon }\end{array}$ \\
\hline
\end{tabular}

Tabelle 1: Klassifikation der Verletzungszonen nach Allen ${ }^{31}$

Einleitung 
Eine weitere Allen-Klassifikation ${ }^{31}$ teilt die Fingerkuppenverletzungen nach Richtung dieser (Abb. 3 und Tab. 2) ein:

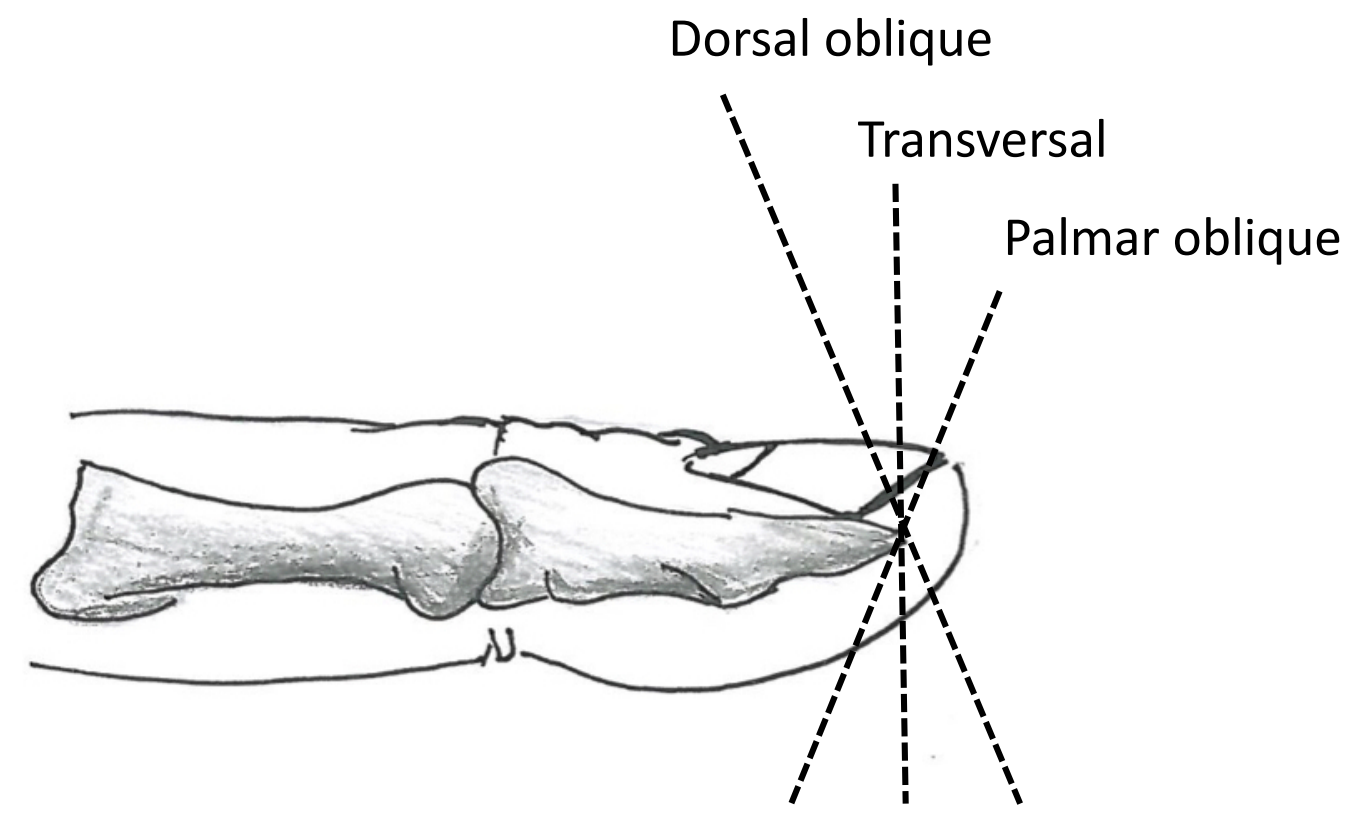

Abbildung 3: Einteilung der Verletzungsrichtungen nach Allen ${ }^{31}$

\begin{tabular}{|l|l|}
\hline Typ I & Transversal = Guillotine Type \\
\hline Typ II & Dorsal oblique = Forward Slope \\
\hline Typ III & Palmar oblique = Backward Slope \\
\hline
\end{tabular}

Tabelle 2: Klassifikation der Verletzungsrichtungen nach Allen ${ }^{31}$

Einleitung 


\subsection{Präoperative Versorgung von Fingerkuppenverletzungen}

Als Erstes sollte bei der notfallmäßigen Vorstellung der Patienten mit Fingerkuppenverletzungen eine kurze Anamnese und Untersuchung erfolgen. Dabei ist es wichtig, Informationen wie Lebensalter, Geschlecht, Beruf, dominante Hand sowie den Unfallhergang bzw. den zeitlichen Rahmen der Verletzung zu erfassen. ${ }^{38}$ Allgemeinerkrankungen wie Diabetes Mellitus, Raynaud Syndrom oder Tabakkonsum können zu Heilungsstörungen führen. Patienten mit Dupuytren-Kontraktur oder mit bestehender Gelenkarthrose sind nach der Rekonstruktion mit lokalen Lappenplastiken gefährdet, postoperative Gelenkkontrakturen zu erleiden.

Bei der Untersuchung der Fingerkuppe sollten der komplette neurovaskuläre Status, Durchblutung, Sensibilität sowie Funktion der Beuge- und Strecksehnen unter Schmerzfreiheit überprüft werden. Die Röntgenbildgebung in zwei Ebenen hilft begleitende Frakturen der Phalanx sowie Fremdkörper auszuschließen.

Eine systematische Evaluation der Fingerkuppenverletzungen besteht aus der Bestimmung von Geometrie und Defektgröße bzw. Vorhandensein freiliegender Knochen und Nagelbettbeteiligung. ${ }^{2}$ Die Fotodokumentation der präoperativen Befunde ist ebenfalls unabdingbar für die vollständige Dokumentation der Untersuchung. ${ }^{39}$ 


\subsection{Unterschiedliche Verfahren zur Defektdeckung}

Der klassische Algorithmus der plastisch-chirurgischen Defektdeckung im aufsteigenden Schwierigkeitsgrad wurde von Majno ${ }^{40}$ im Jahr 1975 als „rekonstruktive Leiter“ in Abbildung 4 dargestellt.

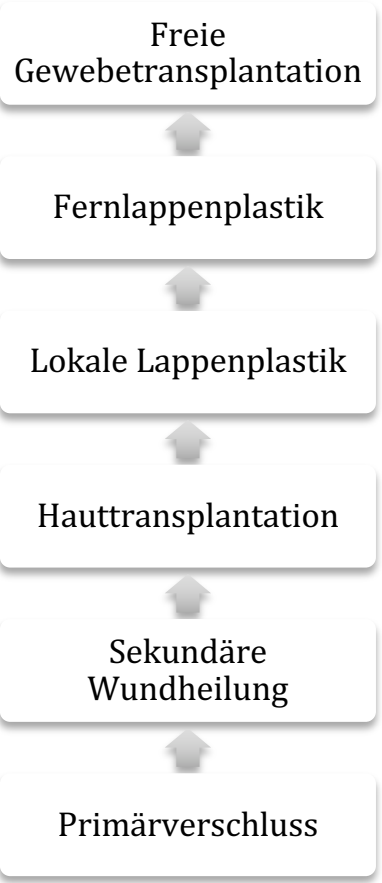

Abbildung 4: Rekonstruktive Leiter der plastisch-chirurgischen Defektdeckung nach Majno $^{40}$

Heute wird die Vorgehensweise situativ in Absprache mit dem Patienten und operativen Möglichkeiten angepasst und nicht mehr am Eskalationsprinzip entschieden. Dieses Prinzip wurde im Jahre 1994 von Gottlieb und Krieger ${ }^{41}$ unter dem Begriff „rekonstruktiver Aufzug" in Abbildung 5 verbildlicht.

Einleitung 


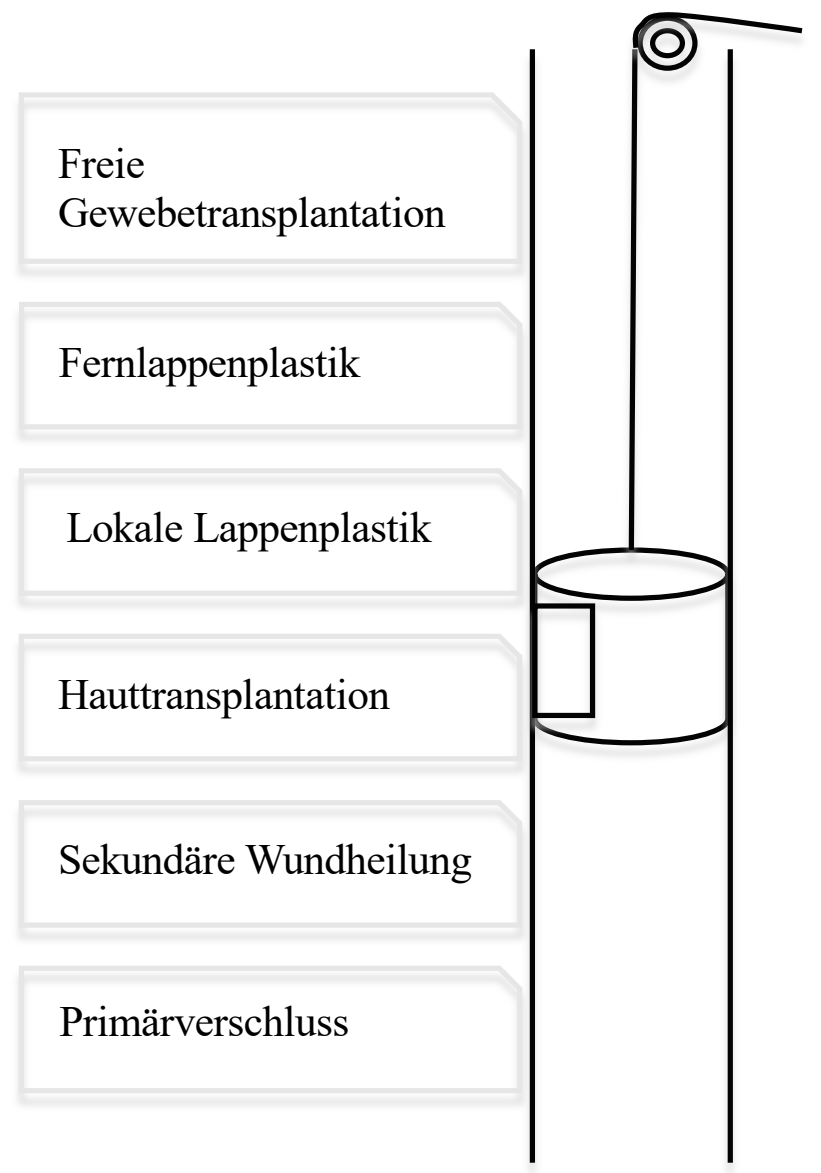

Abbildung 5: Rekonstruktiver Aufzug der plastisch-chirurgischen Defektdeckung nach Gottlieb und Krieger ${ }^{41}$

Ziel der Rekonstruktion ist eine sensible und stabile Fingerkuppe, die die volle Funktion im Rahmen des Greifvorgangs und der Feinmotorik zulässt. Es ist genauso wichtig, eine ausreichende Finger- und Nagellänge, Schmerzfreiheit sowie ein akzeptables kosmetisches Ergebnis zu erzielen. Eine Minimierung der Genesungsdauer und Arbeitsunfähigkeit ist ebenso von großer Bedeutung. ${ }^{42}$

Die suffiziente Rekonstruktionsplanung der Fingerkuppe sollte individuell und unter Betrachtung von Patientenzusammenarbeit, Beruf, Alter, Nebenerkrankungen und Nikotinabusus erfolgen.

Einleitung - 19 - 
Eine fehlerhafte Defektdeckung kann sich bspw. in Form von Sensibilitätstörungen, Nageldeformität- und Neurombildung oder Gelenksteifigkeit manifestieren.

Ein weiterer nicht zu vernachlässigender Punkt ist die Aufklärung der Patienten über mögliche Behandlungsoptionen, deren Folgen und die Rehabilitationsverläufe.

- Konservative Behandlung

Kleine unter $1,5 \mathrm{~cm}^{2}$ große Fingerkuppendefekte bzw. Zone I und II nach der Allen Klassifikation ohne Knochenbeteiligung können konservativ durch geführte Spontanheilung oder Primärverschluss unter Lokalanästhesie direkt in der Notfallambulanz versorgt werden. $^{31}$

Die Komplikationen in Form von Überempfindlichkeit und Kälteintoleranz wurden in ca. 42 bis $51 \%$ beschrieben. ${ }^{43,44}$

In Abhängigkeit der ausgewählten Methode kann eine geführte Spontanheilung durch einen nicht okklusiven Wundverband mit Silver Sulfadiazin, ${ }^{45}$ Fettgasverband, nicht adhärente sterile Kompressen ${ }^{2}$ oder durch Vakuumtherapie ${ }^{46}$ unterstützt werden. In der Nachuntersuchungsstudie von $\mathrm{Lee}^{47}$ und Kollegen konnte eine hohe Patientenzufriedenheit nachgewiesen werden. Zur den Nachteilen gehört eine längere Heilungsdauer von ca. fünf Wochen. ${ }^{48}$ In manchen Fällen sind suboptimale kosmetische Ergebnisse ${ }^{2}$ sowie die Erforderlichkeit einer Patientencompliance beschrieben.

Fingerkuppenamputationen und Quetschverletzungen distal der Insertion der Beugesehne der Zone I bis III nach Allen Klassifikation mit oder ohne Endgliedfraktur bzw. Nagelbeteiligung können nur zum Teil konservativ bspw. mittels Folienverband behandelt werden. ${ }^{49-53}$ Eine bestimmte Fingerkuppenverletzung des Typs IV nach Allen kann mit Semiokklusionsverband versorgt werden. ${ }^{7,54,55}$ Ausnahmen stellen ein palmar liegender und schräg verlaufender Weichteildefekt der Zone II nach Allen sowie Verletzungen mit freiliegenden Sehnen, welche möglichst mit einem Insel-Lappen versorgt werden sollten, dar. ${ }^{2,37,56,57}$ Bereits im Jahr 1962 bestätigt Georg Winter ${ }^{58}$ experimentell, dass eine offene Wunde unter Semiokklusionsverband schneller als unter trockener Behandlung heilen kann. ${ }^{59}$ Auch ist die Infektionsgefahr unter dem Okklusionsverband geringer als unter klassischem Verband. ${ }^{60}$ Grundprinzip der Folientherapie ist eine feuchte Wundbehandlung Einleitung 
unter Retention von Wundsekret. Infolgedessen werden Chemotaxis, Migration, Mitose und Angiogenese aktiviert und ein physiologisches Milieu sowie ein $\mathrm{pH}-$ Wert für Zellwachstum sichergestellt. ${ }^{61-63}$ In Abhängigkeit von Wasserdampftransmissionsrate (WVTR) werden Folienverbände auf okklusiv, semiokklusiv, hochpermeabel und niederpermeabel eingeteilt. De Boer und Collinson ${ }^{64}$ behandeln 1981 mit dem Okklusionsverband zuerst nur Hautdefekte und konnten kürzere Heilungs- und Arbeitsunfähigleitsdauer zur Kontrollgruppe beweisen. Die spätere Weiterentwicklung des Okklusionsverbandes und Verwendung bei Fingerkuppenverletzungen wurde in der Arbeit von Mennen und Wiese ${ }^{51} 1993$ publiziert. Der Verbandwechsel erfolgt einmal pro Woche und kann beim niedergelassenen Chirurgen durchgeführt werden. Es wird keine Ruhigstellung des Fingers benötigt sowie im Vergleich zur operativen Versorgung kein Hebestelledefekt entsteht. ${ }^{65} \mathrm{Zu}$ den weiteren Vorteilen zählen die gute Sensibilität sowie hervorragende kosmetische Ergebnisse.

Die Notwendigkeit der Patientencompliance, Geruchsbildung, ${ }^{65}$ Entstehung von Hypergranulationsgewebe und oft hohe Kosten der Folienverbände sind als Nachteile der Semiokklusionsverbandes zur erkennen. ${ }^{49}$

- Operative Behandlung

Die Fingerkuppenverletzungen mit einem Weichteildefekt ab ca. $1,75 \mathrm{~cm}^{2}$, großflächig freiliegendem Knochen und/ oder Sehnen sowie mit einer Fraktur der Endphalanx sollten möglichst operativ versorgt werden, damit die Fingerkuppe suffizient rekonstruiert werden kann. ${ }^{7}$

\subsubsection{Fingerkuppenamputation}

Als Indikationen für eine Amputation und Stumpfbildung sind Fingerkuppenverletzungen mit Knochenbeteiligung distal sowie proximal der Lunula und transversale Defekte ohne die Möglichkeit einer eigenen Gewebereplantation. Dazu gehören unter anderem stark verschmutzte Wunden sowie Bisswunden. Die Arbeitsprinzipien bei der Amputation sind ein gründliches Debridement, Schaffung der glatten Wundoberfläche, Ableitung der distalen Nerven $1 \mathrm{~cm}$ proximal des Wundrandes, um einer Neurombildung vorzubeugen. Kauterisation der digitaler Arterien bzw. Venen ist für die Prävention von Einleitung 
Hämatomentstehung essentiell. ${ }^{66}$ Eine suffiziente Weichteildeckung der Fingerkuppe und eine postoperative Abhärtungstherapie sollte möglichst angestrebt werden. Die Fingerkuppenamputation ist eine schnelle operative Möglichkeit zur Versorgung von Fingerkuppenverletzungen. $\mathrm{Zu}$ den häufigsten Komplikationen werden Kälteintoleranz und Schmerzanfälligkeit gezählt.

\subsubsection{Hauttransplantation}

Als Alternative kann bei Verletzungen der Zone I nach Allen Klassifikation mit erhaltenem subkutanen Fettgewebe eine Spalthaut- oder Vollhauttransplantation zur Defektdeckung verwendet werden. ${ }^{6}$ Seit der weitgehenden Verbreitung von Okklusionsverbänden sowie der Morbidität der Spenderzone wird eine Hauttransplantation ausschließlich bei sehr großen Defekten, oder wenn andere Verfahren zur Defektdeckung kontraindiziert sind, durchgeführt. ${ }^{11}$ Die dünnen und dicken Spalthauttransplantate können von der Hypothenarregion gewonnen werden. ${ }^{67}$ Zur Versorgung von größeren Wunden ist aufgrund der stärkeren Dehnungsfähigkeit ein dünnes Spalthauttransplantat besser geeignet. Als Komplikationen sind Kälteintoleranz in 33 bis $66 \%$ sowie eine schlechtere Sensibilitätsqualität in 67 bis $76 \%$ im Vergleich zu anderen rekonstruktiven Verfahren beschrieben. ${ }^{68-71}$

\subsubsection{Lokale Lappenplastiken}

Die Rekonstruktion der Fingerkuppenverletzungen mit lokalen Lappenplastiken weist eine hohe Stabilität der rekonstruierten Fingerkuppe durch eine ähnlichere Struktur der Fingerkuppengewebe auf.

- Palmare VY-Dehnungslappenplastik nach Tranquilli-Leali oder Atasoy

Palmare VY-Dehnungslappenplastik oder Verschiebelappen wird häufig zur Rekonstruktion bei schräg dorsal- sowie querverlaufenden Fingerkuppendefekten mit Beteiligung von Knochen und Nagelbett verwendet. ${ }^{72,73}$

Einleitung 
Erstmals wurden VY-Lappen in Form eines Dreiecks durch Tranquilli-Leali ${ }^{74} 1935$ beschrieben, 1970 verbreiten Atasoy ${ }^{75}$ und Kollegen diese Technik in Form von neurovaskulärer Präparation.

Nach Debridement von avitalem Gewebe und Knochenverkürzung erfolgt eine V-förmige Inzision der palmaren Fingerhaut und eine Durchtrennung der Bindegewebssepten sowie eine Verschiebung des Lappens nach distal.

In der Literatur werden exzellente kosmetische Ergebnisse und normale Sensibilität beschrieben. Zu den Komplikationen zählen Berührungsempfindlichkeit in 50 bis $64 \%$ der Fälle sowie Kälteintoleranz, Hypästhesie, Gelenksteifigkeit sowie Krallennagelbildung. ${ }^{675-}$ 78

- Bilaterale VY-Dehnungslappenplastik nach Kutler

Geissendörfer ${ }^{79} 1943$ verwendet bei der Defektdeckung der Fingerkuppe eine unilaterale und Kutler ${ }^{80} 1947$ zwei bilaterale dreiecksförmige kleine Lappen, die von der radialen und ulnaren Seite der Fingerkuppe abgehoben und in der Mitte des Defekts miteinander vernäht werden. Infolge dieser Technik entsteht oft eine radiale Narbe an der Fingerspitze, was zur ausgeprägten Berührungsempfindlichkeit und zur Kälteintoleranz führt. ${ }^{44,81}$

- Palmare Dehnungslappenplastik nach Moberg für Daumenrekonstruktion

Palmare Vorschublappen nach Moberg ${ }^{82}$ von 1964 werden hauptsächlich bei schräg zur Streckseite verlaufenden bis zu $2 \mathrm{~cm}^{2}$ großen Defekten an der Daumenbeere angewendet (Abb. 6). ${ }^{83}$ Eine exzellente Gefäßversorgung wird durch die Verwendung von autonomen dorsalen Arterien des Daumens gewährleistet. Rohrich und Antrobus ${ }^{84}$ beschreiben folgende Vorteile dieser Rekonstruktion: sofortige Wiederherstellung der Sensibilität, Operation in einem Schritt, Erhaltung ursprünglicher Fingerlänge und kortikale Konstanz.

O`Brien ${ }^{85}$ modifiziert 1968 palmare Dehnungslappenplastik nach Moberg in eine InselLappenplastik.

Eine Ruhigstellung der Gelenke nach der Operation soll nicht länger als zehn Tage andauern. Zur Beugung von einer Kontrakturbildung kann eine bilaterale Z-Plastie der Lappenbasis oder proximaler VY-Schnitt verwendet werden. ${ }^{86,87}$

Einleitung 

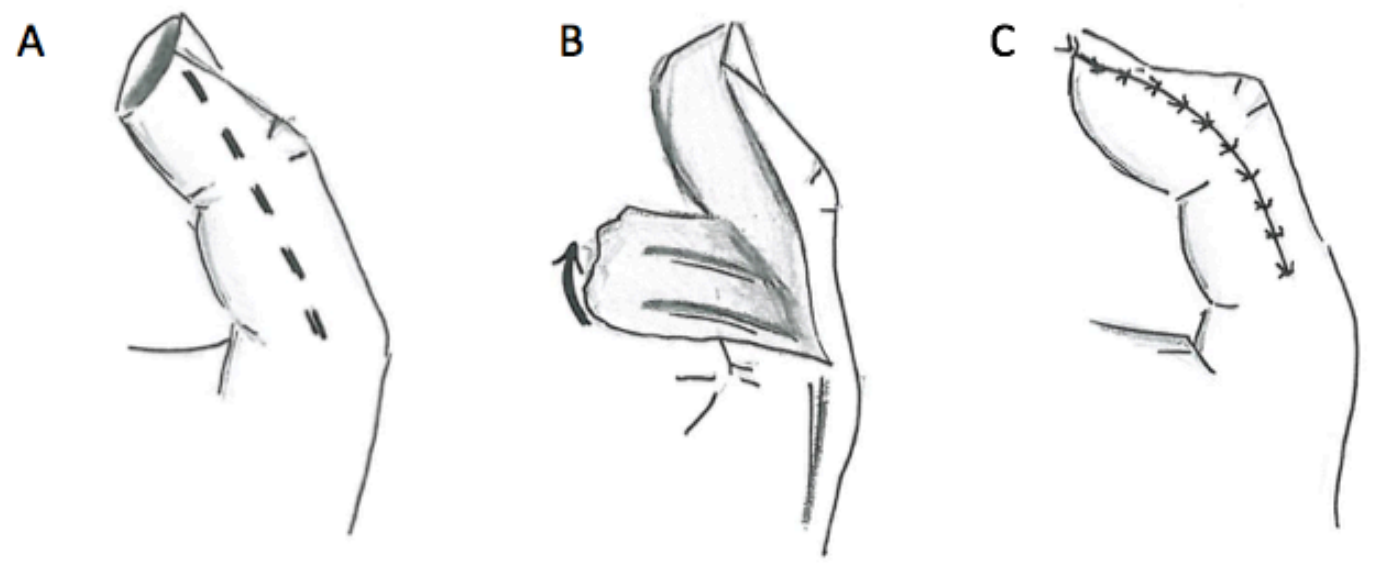

Abbildung 6: Operationsschritte der palmaren Dehnungslappenplastik nach Moberg ${ }^{82}$

A: Mediolateraler ulnarer und radialer Schnitt dorsal der Gefäß-Nerven-Bündel

B: Präparation der Lappen nach proximal zur Beugesehne und durch MCP-Gelenk. Verschiebung der Lappen nach distal

C: Naht der Lappenplastik unter leichte Flexion in IP- und MCP-Gelenk

- Unilaterale Dehnungslappenplastik nach Venkataswami und Subramian

Unilaterale Dehnungslappenplastik nach Venkataswami und Subramian ${ }^{88}$ werden bei transvensalen großen Fingerkuppendefekten mit einer zusätzlichen Verletzung des mediolateralen Fingerkuppenanteils verwendet. Hierbei wird der Lappen mit einem unilateral unversehrten Gefäß-Nerven-Bündel versehen. Bei diesem Verfahren spielt die großzügige Präparation für eine optimale Beweglichkeit und Durchblutung des Lappens eine wichtige Rolle. ${ }^{22,89}$

- Dehnungslappenplastik nach Hueston

Die palmare oder dorsale Dehnungslappenplastik nach Hueston ${ }^{90}$ aus dem Jahr 1966 dient zur Versorgung von kleinen Defekten mit freiliegenden Streck- oder Beugesehnen. Diese entspricht einer Dehnungs-Rotations-Lappenplastik mit einem unilateralen Gefäß-NervenBündel. Die Hebedefektdeckung erfolgt durch Vollhauttransplantation., ${ }^{6,91}$

Einleitung 
- Fernlappenplastiken

Heterotope, aus einer fremden Region entnommene, Haut und subkutanes Gewebe besitzen im Vergleich zur ursprünglichen Haut der Fingerkuppe keinen septalen Aufbau sowie einen unzureichenden Tastsinn. Es führt oft zu mangelndem Widerstand gegen Scherkräfte, Sensibilitätsstörungen und es besteht die Notwendigkeit der kortikalen Neuorientierung, was oft für die älteren Patienten schwierig zu erlernen ist. Eine darauffolgende Notwendigkeit der Defektdeckung am Spenderareal und deren erzwungene Morbidität sollte auch bedacht werden.

- Thenarlappenplastik

Gatewood ${ }^{92}$ beschreibt 1926 die Rekonstruktion von palmaren Fingerkuppendefekten D II D IV mit freiliegender Sehne oder Knochen mit Hilfe der Thenarlappenplastik. Der präparierte Thenarlappen wird direkt auf die Fingerspitze angesetzt. Vorteile dieser Deckungsmethode sind die geringe Morbidität der Spenderfinger und gute kosmetische Ergebnisse. Die Deckung des Hebedefekts kann sofort mit Spalthaut erfolgen. Der Finger sollte zwei bis drei Wochen in Beugestellung fixiert bleiben, was zur Beugekontraktur bei Arthrose oder einem Gelenkschaden führen kann.

- Cross-Finger-Lappenplastik

Die Cross-Finger-Lappenplastik (Abb. 7) sowie die reversed Cross-Finger-Lappen sind von Gurdin und Pangman ${ }^{93} 1950$ erstmals bei palmaren bzw. dorsalen Fingerkuppendefekten in den Zonen II und III mit freiliegenden Sehnen angewendet worden.

Einleitung 


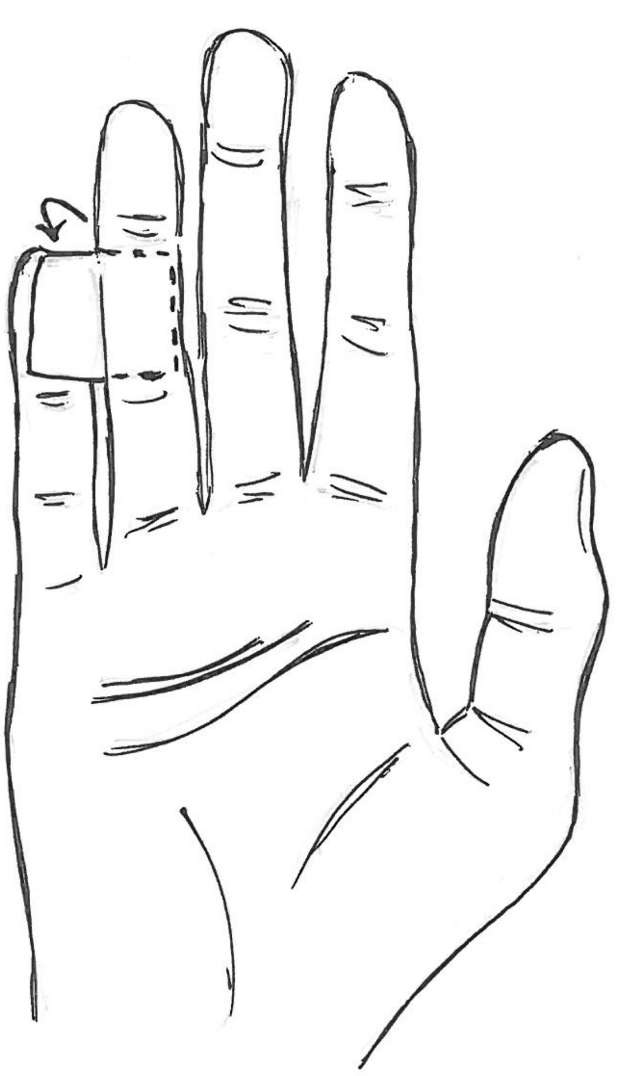

Zuerst erfolgt eine streckseitige Vollhautlappenhebung aus der Mittelphalanx vom ulnarliegenden Nachbarnfinger. ${ }^{6,91,94}$ Der Lappen bleibt radial am Spenderfinger gestielt und wird erst nach ca. drei Wochen, bei der zweiten Operation, abgelöst. ${ }^{95}$ Die Hebedefektdeckung erfolgt durch Spalt- oder Vollhauttransplantation.

Nachteile der Cross-Finger-Lappenplastik sind: Spendermorbidität am Nachbarfinger, Notwendigkeit mehrerer Operationen, Wundheilungsstörungen, Auftreten einer Beugekontraktur in den beiden Fingern nach der postoperativen Ruhigstellung, ${ }^{96}$ Kälteintoleranz in $63 \%{ }^{97}$ sowie unzureichende Sensibilität und taktile Gnosis. ${ }^{98-100}$

Abbildung 7: Cross-Finger-Lappenplastik

- Littler-Lappenplastik

Littler ${ }^{101}$ beschreibt 1956 am Gefäß-Nerven-Bündel gestielte nach ihm benannte LittlerLappen. Mit diesen Lappen können großflächige palmare Daumenkuppendefekte rekonstruiert werden.

Dies sind neurovaskuläre Insel-Lappen, welche von der ulnaren Seite des Mittel- oder Ringfingers gehoben werden. Wegen der gemeinsamen Innervation D I und D II mittels N. medianus wird der Lappen aus dem Mittelfinger als Spenderzone gewählt. Es ist wichtig vor der Operation eine ausreichende Durchblutung der Spender- und Nachbarfinger mit dem Allen-Test oder einer Duplexsonographie abzusichern, da bei der Präparation von GefäßNerven-Bündeln die Arterie des Nachbarfingers geopfert wird..$^{91}$

Einleitung 
- Foucher-Lappenplastik

Die Erstbeschreibung von neurovaskularen Insel-Lappen für die Deckung von ulnaren Fingerkuppendefekten des Daumens mit freiliegenden Sehnen oder Gelenken fand 1950 durch Hilgenfeldt ${ }^{102}$ und 1963 durch Holevich ${ }^{103}$ statt. Später, im Jahre 1979, modifizierte Foucher ${ }^{104}$ diese Technik.

Der Daumen besitzt eine separate dorsale arterielle Versorgung, deswegen kommt es bei der Fingerkuppenrekonstruktion seltener zur Weichteilnekrose als bei Längsfingern.

Hierbei werden A. metacarpalis dorsalis und ein radiodorsaler Ast der N. radialis als GefäßNerven-Bündel verwendet. ${ }^{105}$ Der Lappen wird von der Streckseite der Grundphalanx des Zeigefingers gehoben. Der Hebedefekt weist oft eine vermehrte Empfindlichkeit vor und kann mit einem Vollhauttaransplantat gedeckt werden. ${ }^{106}$

Aufgrund von fehlendem subkutanem Gewebe am Donorlappen ist oft die Füllung der Daumenpulpa unzureichend.

\subsubsection{Fingerkuppenreplantation}

Fingerkuppenreplantation wird bei allen Verletzungstypen, außer bei Quetsch-, Ausrissdefekten oder bei mangelhafter Gefäßversorgung, indiziert. In der Literatur liegt die Überlebensrate des Replantates zwischen der Nagelfalte und dem IP-Gelenk bei 70 bis $86 \% .{ }^{107,108} \mathrm{Da}$ es sich um eine mikrochirurgische Rekonstruktion handelt, wird eine gute Infrastruktur der Klinik benötigt und es ist mit einer langen Operationsdauer bzw. Nachoperationen zu rechnen. Operativ werden Anastomosen zwischen Arterien sowie ein venöser Abfluss hergestellt, ohne digitale Nerven zu rekonstruieren. Vorteile hierbei sind, dass die ursprüngliche Länge des Fingers sowie die Ästhetik, beibehalten wird. Von dieser Technik profitieren besonders junge Patienten und Berufsgruppen wie Musiker.

Als unerwünschte Folgen können Kälteintoleranz, Sensibilitäts- und Nagelwachstumsstörungen, Pseudoarthrosen sowie Pulpaatrophien auftreten. ${ }^{6,22,109-111}$

\subsubsection{Freie mikrochirurgische Gewebetransplantation}

Die mikrochirurgische Zehenpulpatransplantation ist für die Daumenamputation im Bereich des MCP-Gelenks und sogar distal des IP-Gelenks hervorragend anwendbar. Bei der Einleitung - 27 - 
Präparation des Zehes werden metatarsale Gefäße am proximalen Rand freigelegt und dann als End-zu-End Anastomose mit A. digitalis ulnaris mikrochirurgisch vernäht. ${ }^{112}$ Trotz großen technischen und operativen Aufwands sowie längeren Rehabilitationszeiten hat sich diese Technik als eine sichere Rekonstruktionsmethode für die Herstellung der Sensibilität des Daumens bzw. der Greiffähigkeit der Hand bestätigt. Herr Del Pinãl beschreibt in seiner Arbeit eine Erfolgsquote von $98,6 \%$ bei der Verwendung von mikrochirurgischen Zehenpulpatransplantationen in der Rekonstruktion von Daumenamputationen. ${ }^{113}$

\subsection{Definition und Entwicklung homodaktylen neurovaskulärer Insel-} Lappenplastiken

Das alte Grundprinzip der Defektdeckung "Gleiches mit Gleichem" wird in der neurovaskulären Insel-Lappenplastik eingesetzt. ${ }^{114}$ Durch die Verwendung von Lappen mit Gefäß-Nerven-Bündeln wird eine bestmögliche Herstellung der Innervation und Durchblutung an der verletzten Fingerkuppe gewährleistet.

Insel-Lappen sind von allen Seiten frei präpariert und nur an einem neurovaskulärem Pedikel angebunden, wodurch eine größere Lappen-Mobilisation gewährleistet wird. ${ }^{115}$

Bei der homodigitalen Lappenplastik wird der freipräparierte Lappen und das Gefäß-NervenBündel vom verletzten Finger gehoben, dagegen wird bei einem heterodigitalen Lappen ein Spenderfinger benötigt.

Die Insel-Lappenplastiken werden abhängig von der Arterienflussrichtung in zwei Kategorien unterschieden:

1. Bei dem anterograden Insel-Lappen wird das Gefäß und der kollaterale Nerv in das gemeinsame Weichteilgewebe eingebettet und der proximale Defektrand bildet den distalen Rand des Lappens. ${ }^{114}$

2. Bei dem retrograden Insel-Lappen werden drei palmare transversale Arterienarkaden sowie Perforansgefäße in der arteriellen Versorgung verwendet, was eine Deckung von größeren Defekten ermöglicht. ${ }^{116}$ 
Im Jahre 1956 publiziert Littler ${ }^{101}$ seine Arbeit in welcher er die Rekonstruktion der Daumenpulpaverletzung durch neurovaskulär gestielte Insel-Lappen aus einem Spenderfinger beschriebt.

Neun Jahre später verwendet Flint und Harrison ${ }^{117} 1965$ sowie Joshi ${ }^{118} 1974$ dorsale Arterienäste des verletzten Fingers als eine homodigitale monopedikuläre InselLappenplastik.

Die Kutler Lappen modifizierten 1976 Segmüller ${ }^{119}$ und 1979 Biddulph $^{120}$ und schlagen eine neurovaskuläre laterale Insel-Lappenplastik vor. Der Lappen ist an zwei Gefäß-NervenBündeln gestillt, was eine größere Beweglichkeit des Lappens und eine Deckung von weitliegenden Verletzungen ermöglicht.

Venkantaswami und Subramian ${ }^{88}$ dokumentieren 1980 eine monopedikuläre homodigitale neurovaskuläre Insel-Lappenplastik in Dreieckform als Therapieoption zur Deckung bei quer-palmaren Fingerkuppenverletzungen. Mouchert und Gilbert ${ }^{121}$ wandten 1982 diese Technik erstmals bei Kindern und Schuind ${ }^{122}$ drei Jahre später auch bei der Erwachsenen an. In den Arbeiten von Lai ${ }^{123} 1989$ und Kojima ${ }^{124} 1990$ wurden retrograde homodaktyle neurovaskuläre Insel-Lappen beschrieben.

Einleitung 
5.6 Vor- und Nachteile neurovaskulärer Insel-Lappenplastiken

\begin{tabular}{|c|c|}
\hline Vorteile & Nachteile \\
\hline $\begin{array}{l}\text { - } \text { Erhaltung der ursprünglichen } \\
\text { - } \text { Fingerlänge } \\
\text { Nur eine Operation (,one stage flap“) } \\
\text { - } \text { Keine Immobilisation oder } \\
\text { Verletzung anderer Handteile } \\
\text { - Möglichkeit der Defektdeckung von } \\
\text { großen distalen } \\
\text { Fingerkuppenverletzungen } \\
\text { - Erhaltung der Sensibilität } \\
\text { - Wiederherstellung der identischen } \\
\text { Hautqualität und Hautfarbe } \\
\text { - Gute ästhetische und funktionelle } \\
\text { Outcome }\end{array}$ & $\begin{array}{l}\text { - } \text { Gefahr der Kontraktur im DIP- } \\
\text { und PIP-Gelenk } \\
\text { - } \quad \text { Gefahr der Lappennekrose }\end{array}$ \\
\hline
\end{tabular}

Tabelle 3: Vor- und Nachteile neurovaskulärer Insel-Lappenplastiken

Einleitung 
5.7 Indikationen und Kontraindikationen neurovaskulärer Insel- Lappenplastiken

\begin{tabular}{|c|c|}
\hline Indikationen & Kontraindikationen \\
\hline $\begin{array}{l}\text { - Defekte der Pulpa in Zone II- } \\
\text { III nach Allen mit } \\
\text { freiliegendem Knochen von bis } \\
\text { zu } 6 \text { x 3,5 cm }\end{array}$ & 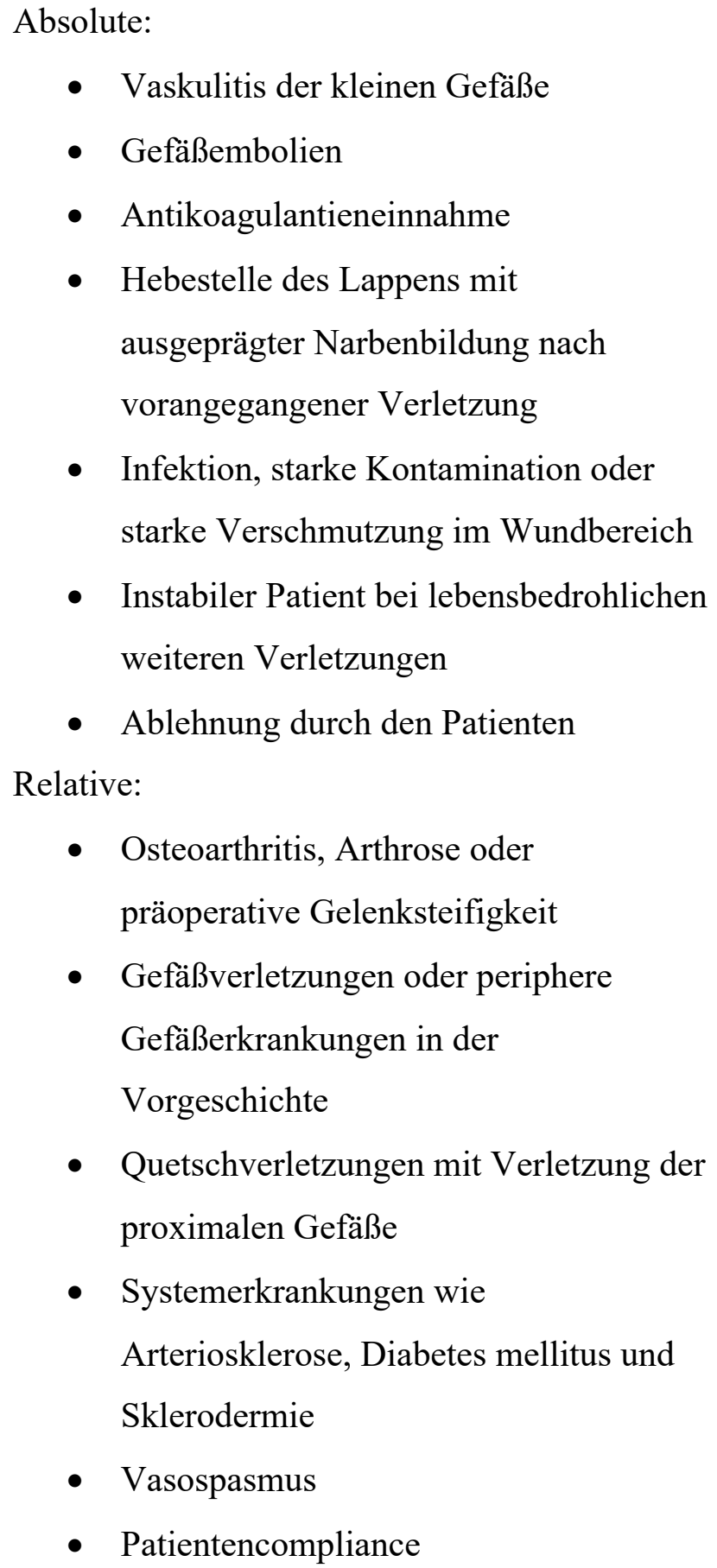 \\
\hline
\end{tabular}

Tabelle 4: Indikationen und Kontraindikationen neurovaskulärer Insel-Lappenplastiken Einleitung $-31-$ 
Aufgrund unzureichender Arterienkaliber wird neurovaskuläre Insel-Lappenplastik bei radialen Zeigerfingerdefekten sowie bei ulnaren Kleinfingerdefekten nicht angewendet.

\subsection{Verlauf der Operationsvorbereitung}

\subsubsection{Patientenaufklärung}

1. Risiken und mögliche Komplikationen nach der Operation

- Allgemeine Operationsrisiken

- Gefahr einer akuten Infektion

- Gefahr einer Lappennekrose

- Wundheilungsstörung

- Gefahr der Entstehung eines komplexen regionalen Schmerzsyndroms

- Narbenbildung sowie Narbenkontrakturentstehung

- Sensibilitätsstörungen und Kälteempfindlichkeit im Fingerkuppenbereich

- Beugekontraktur im PIP-Gelenk

- Gefahr einer Neurombildung

2. Behandlungs-, Rehabilitation- und Arbeitsunfähigkeitsdauer

\subsubsection{Operationsvorbereitung}

- Anamnese

- Klinische Untersuchung mit Inspektion, Palpation und Funktionsprüfung

- Röntgenaufnahme des betroffenen Fingers um eine knöcherne Verletzung bzw. ein Fremdkörper auszuschließen

- Ggf. Allen-Test der Finger

- Wundreinigung und Wundkonditionierung

- Erstellung der Defektschablone

\subsubsection{Instrumentarium}

- Steriler Stift

- Lupenbrillenvergrößerung 
- Handchirurgisches Instrumentarium (z. B. Skalpell Große 11 zur Hautinzision, scharfe Schere zur Präparation des Lappens, Hauthaken, chirurgische Pinzette, Nadelhalter)

- Bipolar

- Monofiler Hautfaden z. B. der Stärke 5/0

\subsubsection{Anästhesie und Lagerung}

- Rückenlage

- Lagerung des Arms auf einem speziellen Handtisch

- Anästhesiemöglichkeiten: Oberst-Leitungsanästhesie, axilläre Plexusanästhesie, Allgemeinanästhesie

- Blutleere durch elastisches Band und Blutsperre des betroffenen Fingers

\subsection{Unterschiedliche Operationstechniken}

\subsubsection{Chirurgische Anatomie}

Das Gefäß-Nerven-Bündel des Fingers verläuft innerhalb subkutanen Gewebes und ist palmar durch Grayson Band und dorsal durch das Cleland Band begrenzt. Bei der Operation wird die komplette Spaltung der Bänder durchführt. Während der Präparation des Nervs werden multiple palmare Nervenäste für die Erhaltung der Beweglichkeit des Gefäß-NervenBündels geopfert.

Anterograde homodigitale neurovaskuläre Insel-Lappenplastik

Technische Einteilung:

1. Stufenleiter Effekt, ohne Hauttransplantat

2. Schmaler Lappen, mit Vollhauttransplantat 


\subsubsection{Operationstechnik mit anterograden homodigitalen neurovaskulären Insel-Lappen}

Abbildung 8 (A-D) zeigt anhand eines Beispiel-Patienten und Schema-Zeichnungen die Operationstechnik mit anterograden homodigitalen neurovaskulären Insel-Lappen. Zum Operationszeitpunkt war der Patient 44 Jahre mit einer Querschnittverletzung und Amputation der rechten Zeigerfingerkuppe mit freiliegendem Knochen.

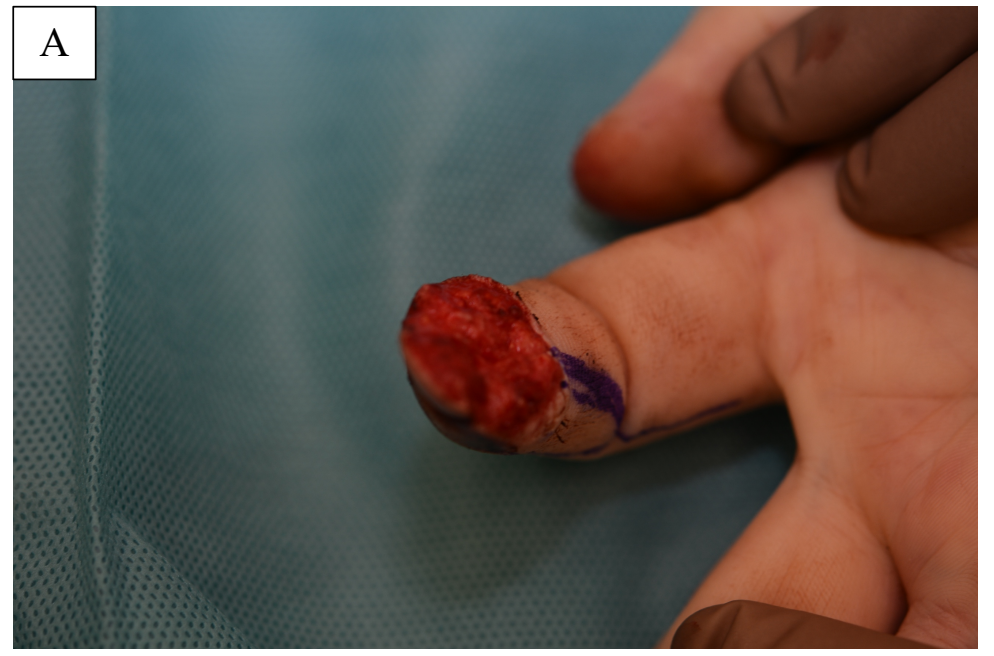

A: Beugeseitig schrägverlaufender Defekt der Fingerkuppe mit freiliegendem Knochen und ohne Beteiligung des Fingernagels. Mit dem sterilen Hautstift Einzeichnen eines lateralen, neurovaskulären Insel-Lappens von ulnar nach radikalem Debridement.

B: Inzision der Haut entlang der eingezeichneten Linie. Vorsichtige Präparation des Insel-Lappens

\section{B}

mittels Präparation

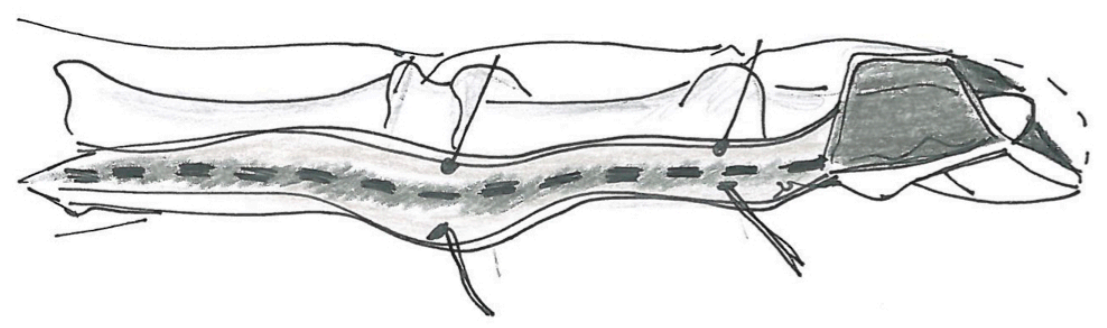

des Hautweichteilmantels vom Lappen weg. Identifizierung und Präparation des GefäßNervenbündels im subkutanen Gewebe. Es ist dafür Sorge zu tragen, dass die Arterie und der Nerv mit ausreichend perivaskulärem Gewebe umgeben ist, was für einen venösen Abfluss sorgt. 


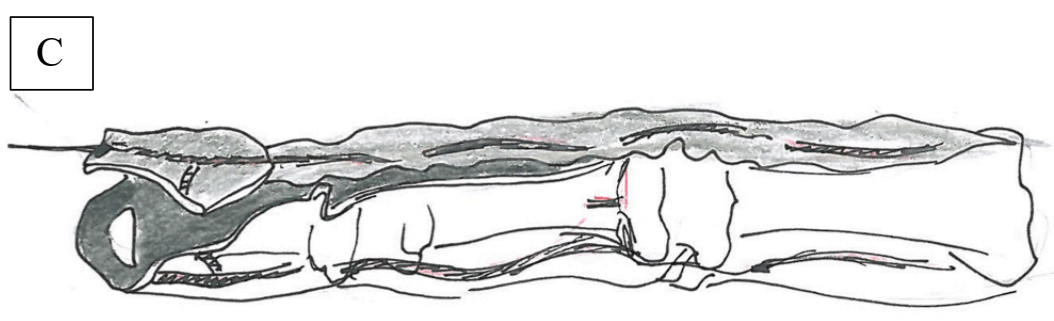

C: Der präparierte Lappen hängt frei am Gefäß-Nerven Bündel. Anschließend mobilisieren des Lappens mit Hautharken nach distal (ggf. unter Beugung im DIP-Gelenk) und Einpassen des Lappens in den bestehenden Defekt mit sicherer Bedeckung des Knochens.

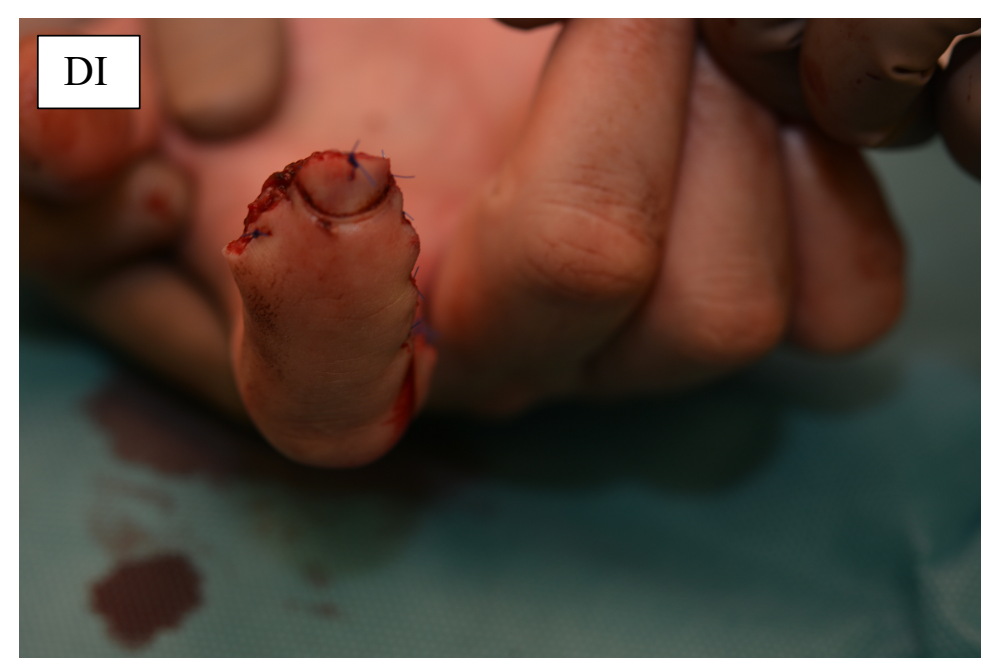

DI/II: Lockerer Verschluss des Hebedefektes mit Hautnaht ohne Komprimierung des GefäßNerven-Bündels sowie Einnähen der Lappen im Bereich der Fingerkuppe mittels monofilen Einzelknopfnähten z.B. der Stärke 5/0.

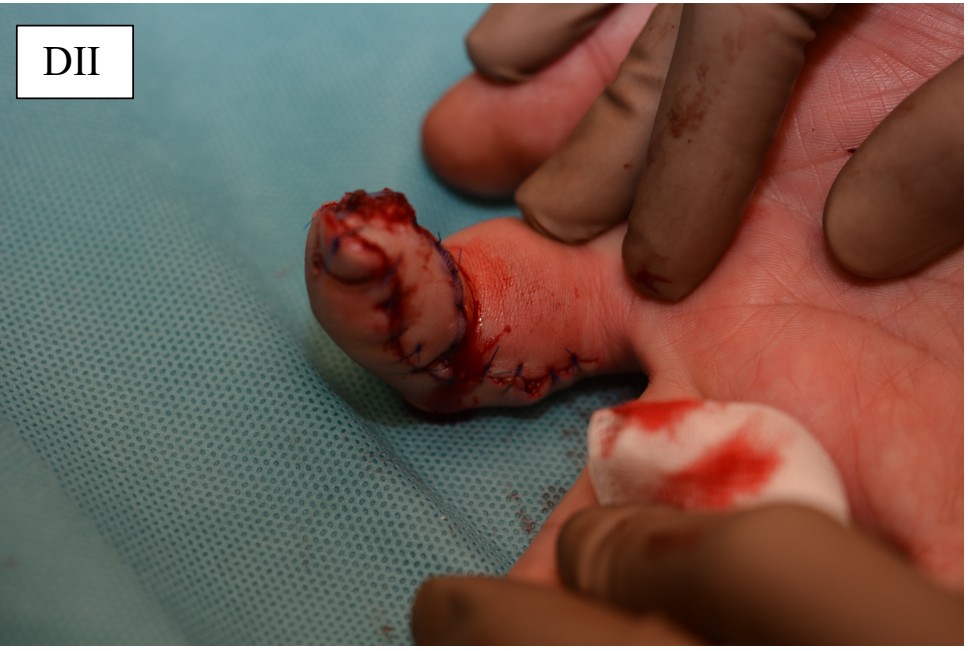

Abbildung 8: Operationsschritte bei anterograden homodigitalen neurovaskulären InselLappen

Einleitung $-35-$ 


\subsubsection{Besonderheiten}

Es ist wichtig, vor der Inzision des Lappens sicherzustellen, dass das Gefäß-Nerven-Bündel innerhalb des geplanten Lappens verläuft. Dabei wird zuerst der medio-laterale Schnitt durchgeführt, um den Stil zu identifizieren. Das erlaubt dem Operateur -falls notwendig- die Lappen umzuplanen sowie die klinische Inspektion des Gefäß-Nerven-Bündels durchzuführen. Wenn ein Verdacht auf Arterieninsuffizienz vorliegt, sollte eine erneute Durchführung des Allen-Tests ggf. eine Dopplersonographie unter Auflösung der Blutleere erfolgen. Bei unzureichender Lappengröße sollten bevorzugt der zentralliegende Pulpadefekt oder die freiliegenden Knochen und Sehnen bedeckt werden. Das Gefäß-Nerven-Bündel wird immer proximal des PIP-Gelenks und in leichter Flexionsstellung des IP-Gelenkes inzisiert. Das ermöglicht die freie Präparation von kleinen Gefäßen, Nerven und ligamentären Strukturen (Grayson und Cleland Band).

\subsubsection{Postoperative Behandlung und Rehabilitation}

- Kontrolle der Hautlappendurchblutung nach Öffnung der Blutsperre

- Steriler Verband (milde Kompressionsstufe)

- Ggf. Entscheidung über Anlage einer dorsal blockierenden Schiene bezüglich der Patientencompliance und der Verletzungsart

- Ambulante postoperative Behandlung

- 1. Woche: Ggf. Stabilisierung durch eine dorsal blockierende Schiene und Flexionssowie Extensionsübungen innerhalb der Schienengrenzen

- 2. Woche: Nahtentfernung. Aktive und passive sowie extensive Übungen

- Ab 3. Woche: Absetzen der Schiene

- Wenn nötig, Anlage einer Extensions-Schiene während der Nacht für eine Korrektur der Beugekontraktur

\subsubsection{Fehler, Gefahren und Komplikationen}

- Beugekontraktur im PIP-Gelenk nach Abnahme des Gipsverbandes: Krankengymnastische Behandlung 
- Totaler oder partieller Lappenverlust in Folge zu fester Verbandsanlage, Torsion oder ggf. übermäßigem Ziehen von neurovaskulärem Pedikel während der Lappenmobilisation

- Perfusion- und Sensibilitätsstörungen durch exzessive Zugspannung des Lappens beim Vernähen: Alternative Defektdeckung zu überlegen

- Infektionen in Ausmaß der septischen Chirurgie

- Neurombildung: Desensibilisierung durch Ergotherapie

- Krallennageldeformität in Folge zu engen distalen Lappenrands oder zu enger Befestigung an der sterilen Nagelmatrix 


\section{Methoden}

Vor Beginn der Studie wurde das Ethikvotum (Zeichen FF61/2016) bei der Ethikkommission der Landesärztekammer Hessen eingeholt und am 07.12.2017 genehmigt.

Alle nachuntersuchten Patienten wurden in der Abteilung für Plastische-, Hand- und Rekonstruktive Chirurgie der Berufsgenossenschaftlichen Unfallklinik Frankfurt am Main operiert.

Bei der Suche von Patienten aus der Datenbank mit dem Stichwort: „Lokale Lappenplastik an Haut und Unterhaut an den Fingern“ wurden initial 138 Patienten im Zeitraum vom 01.01.2003 bis 31.12.2014 ermittelt. Nach der Aktenrecherche verblieben 53 Patienten mit 57 Fingerkuppenverletzungen, welche im Zeitraum vom 22.04.2003 bis 01.09.2014 mit einer neurovaskulären Insel-Lappenplastik operiert wurden und allen Einschlusskriterien entsprachen. Die anderen 85 Patienten wurden mit Cross-Finger Lappen, Thenarlappen, VY-Verschiebelappen und Kutler-Lappen versorgt.

Aus den 53 in die Studie aufgenommenen Patienten wurden 23 (43,4 \%) ausgeschlossen: 16 Patienten (69,6\%) waren unbekannt verzogen, 6 (26,0\%) telefonisch sowie per Anschreiben nicht erreichbar oder wegen Mangel an persönlichen Daten nicht auffindbar. Bei einem Patienten wurde infolge einer Nekrose und Infektion eine Amputation im DIP-Gelenk durchgeführt mit dem Verlust der Insel-Lappen.

Von den 30 Patienten (56,6 \%), welche wir erreichen konnten, waren lediglich zwei Patienten $(6,6 \%)$ wegen längeren Arbeitszeiten und mangelnder Kooperation nicht bereit, an der Studie teilzunehmen.

Von insgesamt 28 eingeschlossenen Patienten (93,3 \%) mit 29 Fingerkuppenverletzungen wurden 24 Patienten (80 \%) im Zeitraum vom 03.01.2018 bis 10.03.2018 ausführlich durch die Fragebögen befragt und klinisch nachuntersucht, davon 23 Patienten durch eine Vorstellung in unserer Klinik und einer per Hausbesuch.

Daraus entstand eine Datenbank mit detaillierten Operationsergebnissen über 25 rekonstruierten Fingerkuppen.

Anschließend konnten wir vier Patienten (20\%) telefonisch erreichen und mit Hilfe eigens erstellter Frage- und Nachuntersuchungsbogen sowie des DASH-Fragebogens befragen.

Methoden

$-38-$ 


\subsection{Einschlusskriterien}

- Fingerkuppenverletzungen, bei denen eine plastisch-chirurgische Defektdeckung mit neurovaskulärem Insel-Lappen notwendig war

- Zum Zeitpunkt der Nachuntersuchung waren alle Patienten volljährig und einwilligungsfähig

- Erhebung von Langzeitergebnissen mit einer Nachbeobachtungszeit von mindestens 36 Wochen

\subsection{Ausschlusskriterien}

- Fingerkuppenverletzungen, die konservativ behandelt wurden

- Fingerkuppenverletzungen im Rahmen eines komplexen Traumas der Hand mit Verletzungen von Gefäßen, Nerven oder Knochen proximal des Endgliedes

\subsection{Untersuchungsdesign}

1. Auswertung der Patientenakten und Erhebung der folgenden Parameter:

- Epidemiologische Daten (Geburtsdatum, Geschlecht, Wohnort, Beruf zum Zeitpunkt des Unfalls, Alter zum Zeitpunkt der Operation)

- Ort des Unfalls

- Grund der Aufnahme

- Operationsdatum

- Dauer des stationären Aufenthalts

- Art der Fingerkuppenverletzung

- Ort der Verletzung (Finger DI bis DV)

- Seite (rechts oder links) der verletzten Hand

- Radial-, ulnar- oder beidseitige Defektdeckung durch neurovaskuläre Insel-Lappen

- Sonstig verletzte Finger

- Notwendigkeit der Osteosynthese

- Nachbehandlung, Verband

- Anzahl der Revisionsoperationen

Methoden 
- Postoperative Komplikationen (Infektion, Nekrose, Nachblutung)

Die Auswertung radiologischer Daten erfolgte retrospektiv anhand der vorhandenen Röntgenbilder im Hinblick auf Verletzungshöhe und, sofern postoperative Bilder vorhanden sind, auf die knöcherne Heilung der Endgliedfraktur. Es entstand keine Strahlenbelastung für die Patienten.

2. Die Einbestellung der Patienten erfolgte telefonisch und per Anschreiben. Die Patienten erhielten eine schriftliche Patienteninformation sowie die Einwilligungserklärung zur freiwilligen Teilnahme an dieser Studie. Die erhobenen Krankheitsdaten wurden für die weitere Auswertung verschlüsselt und pseudonymisiert.

3. Befragung der Patienten mit Hilfe eigens erstellter Frage- und Nachuntersuchungsbögen (siehe Anhang):

- Epidemiologische Daten (Alter, Geschlecht, Beruf, Händigkeit, etc.)

- Vorerkrankungen und Medikamente

- Raucheranamnese

- Arbeitsunfähigkeitsdauer

- Dauer der endgültigen Heilung

- Vorhandensein von freiliegenden Knochen

- Art der Nachbehandlung und Verbandswechsel

- Anzahl der Physio- und Ergotherapieeinheiten

- Probleme an der Entnahmestelle

- Beschreibung der Narbe mit Hilfe der Vancouver Scar Scale (VSS)

Die Vancouver Scar Scale ${ }^{125}$ (VSS) wurde ursprünglich zur Evaluation von Verbrennungsnarben ${ }^{126} 1990$ im General Hospital in Vancouver erstellt und dient heute als standardisierte Skala zum Assessment von verschiedenen Narbenarten. ${ }^{127,128}$ Die Skala erfasst vier objektive Narbeneigenschaften: Pigmentierung, Durchblutung, Geschmeidigkeit und Höhe der Narbe.

Der Gesamtpunktzahl der einzelnen Punkte reicht von 0 bis maximal 13 Punkte, 0 Punkte entsprechen annähend der normalen Haut. 
- Erfassung der postoperativen Schmerzen in Ruhe und unter Belastung mit der numerischen Rating-Skala von 0 bis10 (Abb. 9)

Die numerische Rating-Skala ${ }^{129}$ (NRS) ist das meist verbreitete und unkomplizierte Instrument zur Erfassung der akuten oder chronisch subjektiven Schmerzempfindlichkeit ${ }^{130,131}$ bei Erwachsenen und kann als horizontale Grafik ${ }^{132}$ verbildlicht oder verbal befragt werden (Abb. 9). Der Patient gibt postoperativ die Schmerzintensität in Punkten von 0 (= kein Schmerz) bis 10 (= maximal vorstellbarer Schmerz) zuerst in Ruhe, danach unter Belastung an.

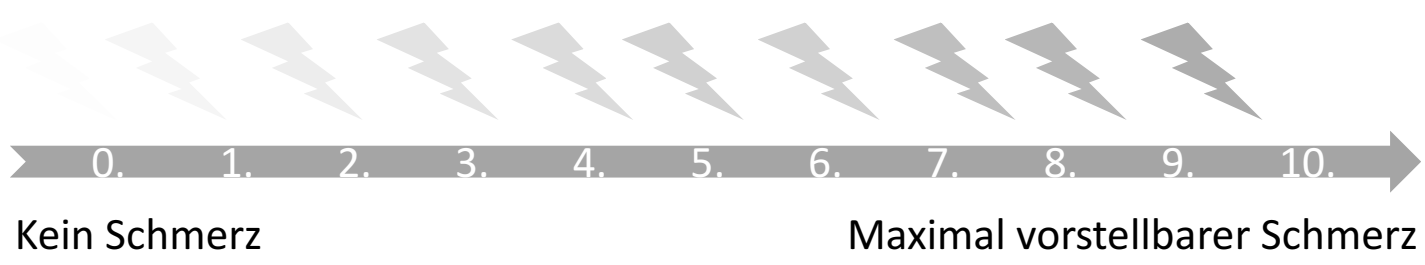

Abbildung 9: Numerische Rating-Skala

- Erfassung von subjektiver und objektiver Temperatur bzw. Schweißbildung

- Erfassung von Neurombeschwerden, Kälteempfindlichkeit, Schwellung sowie Druckschmerz

- Überprüfung von Vorhandensein von Hoffmann-Tinel-Zeichen ${ }^{133}$ durch Beklopfen der Fingerkuppe

Hoffmann-Tinel-Zeichen beschreibt ein elektrisierendes Gefühl bzw. Parästhesien im Versorgungsgebiet eines geschädigten Nervs als subjektives Empfinden, teils tritt es beim Beklopfen der Hautoberfläche auf. ${ }^{134}$ Dadurch werden Rückschlüsse auf eine fehlende Nervenregeneration oder schlechte Erholungsfähigkeit nach einer Nervenverletzung gezogen. Dieses Zeichen wird auch als Diagnosekriterium einer peripheren Neuropathie benutzt.

- Anzahl der Revisionsoperationen intern

- Anzahl der Revisionsoperationen extern

- Postoperative Komplikationen (Infektion, Nekrose, Nachblutung)

Methoden 
- Subjektive Patientenzufriedenheit anhand des erstellten Fragebogens mit Hilfe der numerischen Analogskala von 0 bis $10(0=$ maximal unzufrieden, $10=$ maximal zufrieden)

- Erfassung der Beschwerden und der Funktionsfähigkeit nach einer Verletzung der oberen Extremität mit dem DASH-Fragebogen ${ }^{135}$ und Berechnung von einem DASH-Score

Der DASH-Fragebogen ${ }^{135}$ ist ein Instrument für die Erfassung subjektiver Empfindung der Untersuchten in Bezug auf Einschränkung der Funktion der oberen Extremitäten. Es werden Auswirkungen auf Aktivitäten des täglichen Lebens (ADL) in den letzten Wochen abgefragt, ebenso fließt die Einschränkung bei Ausübung von Arbeit, Sport oder Musik in den Score mit ein. Die Befragung bezieht sich auf die Ausübung der Tätigkeit mit beiden Extremitäten. Der DASH-Fragebogen wurde 1996 gemeinsam von dem "Council of Muskuloskeletal Speciality Societies", dem "Institute for Work and Health" und der "American Academy of Orthopedic Surgeons" erarbeitet. Bei mindestens 27 von $30 \mathrm{zu}$ beantworteten Fragen wird ein DASH-Score berechnet: 0 steht für keine Beeinträchtigung der Funktion, 100 für eine extreme Beeinträchtigung.

$$
\text { DASH-Score }=\left(\frac{\text { Gesamtpunktzahl }}{\text { Anzahl der beantworteten Fragen }}-1\right) \times 2
$$

4. Nachuntersuchung der Patienten

Klinische Daten und Diagnostik:

- Lappengröße (Länge und Breite)

- Messung der Bewegungsumfänge mit Standart-Goniometer nach der Neutral-NullMethode am Handgelenk, betroffenen Finger bzw. Daumen sowie der Gegenseite:

- Flexion/Extension, Radialduktion/Ulnarduktion sowie Supination/Pronation des proximalen und distalen Handgelenks (Abb. 10 bis 12)

Methoden 


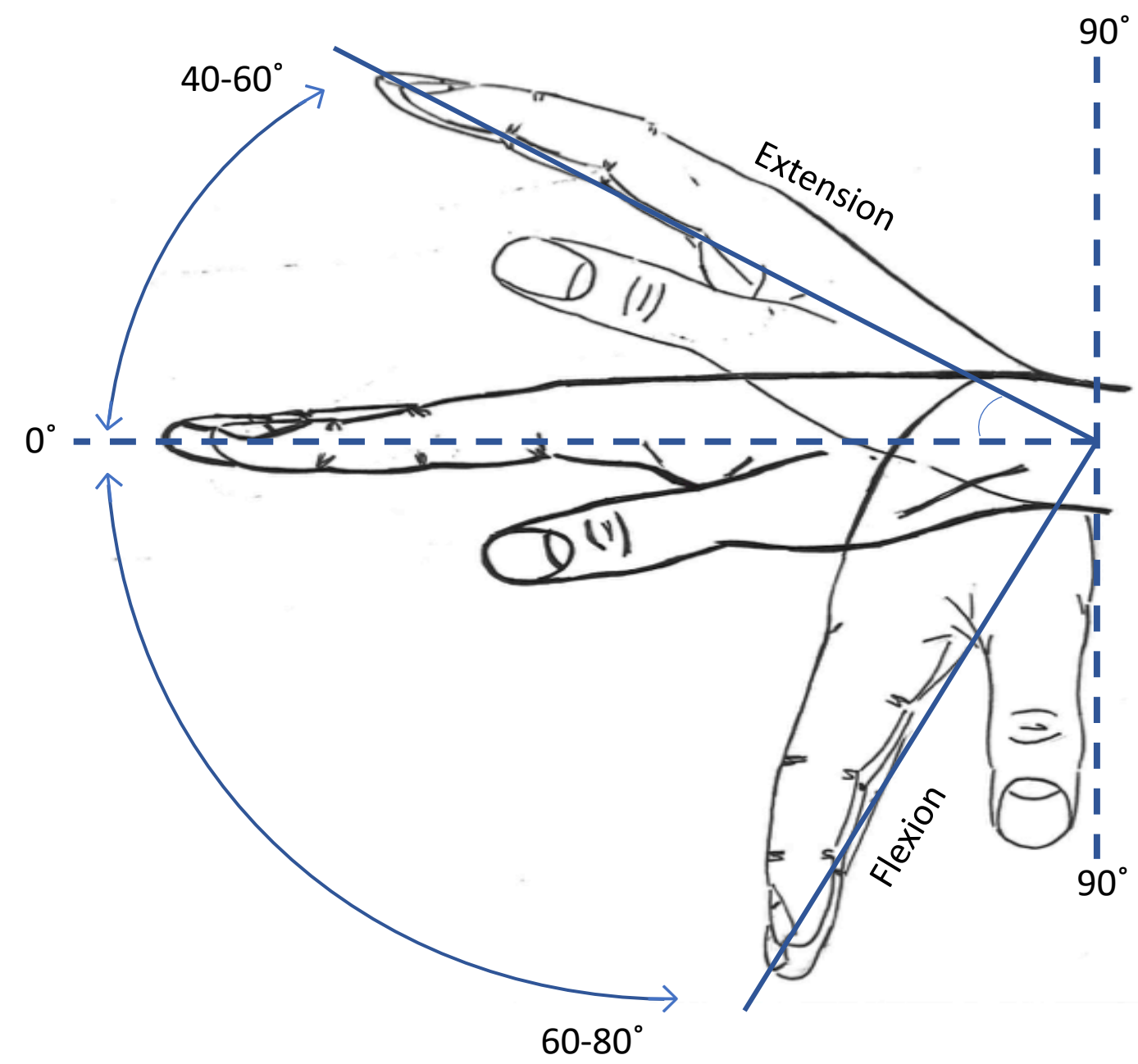

Abbildung 10: Messung der Extension und Flexion des Handgelenks

Methoden

$-43-$ 


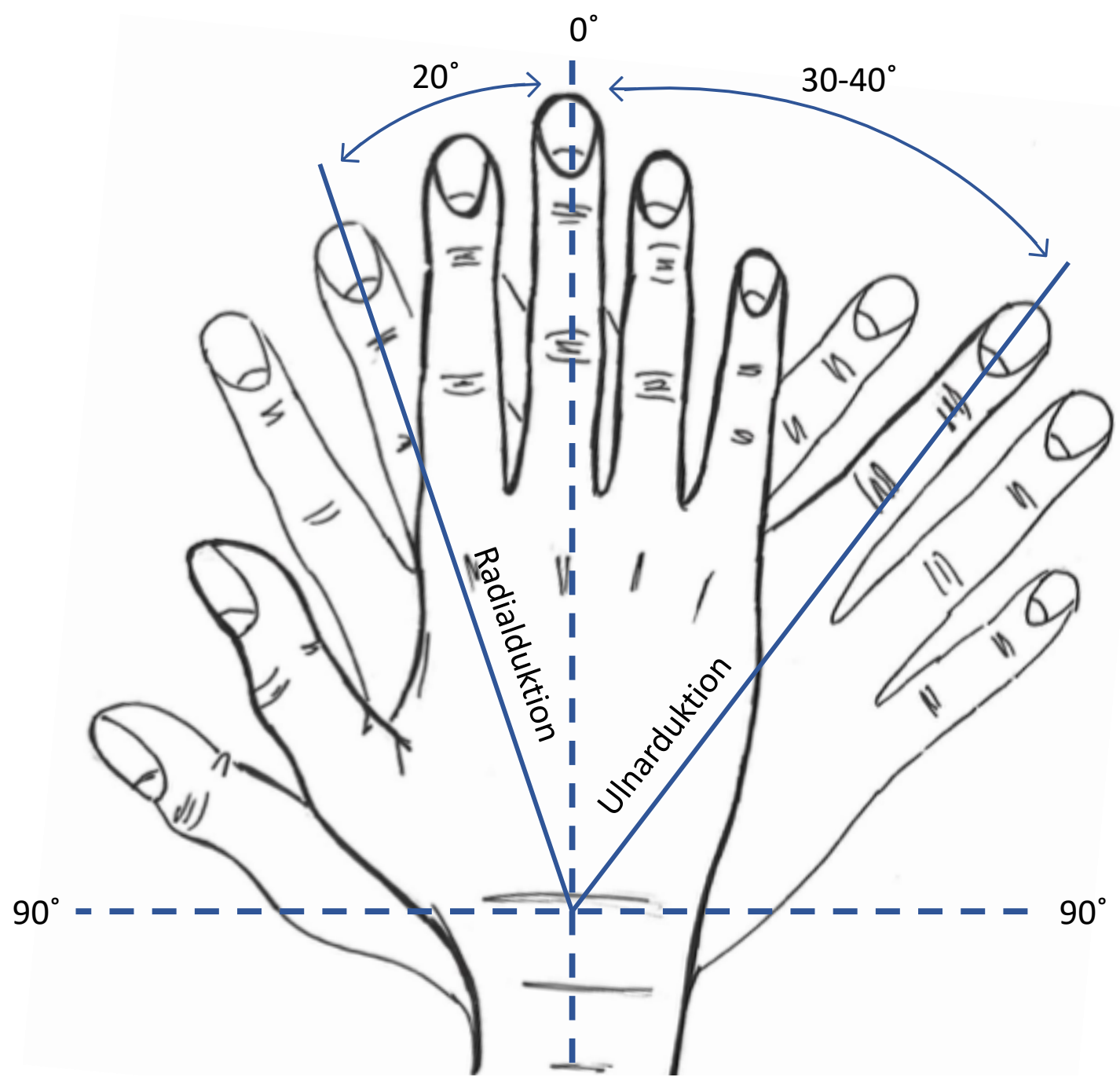

Abbildung 11: Messung der Radialduktion und Ulnarduktion des Handgelenks 


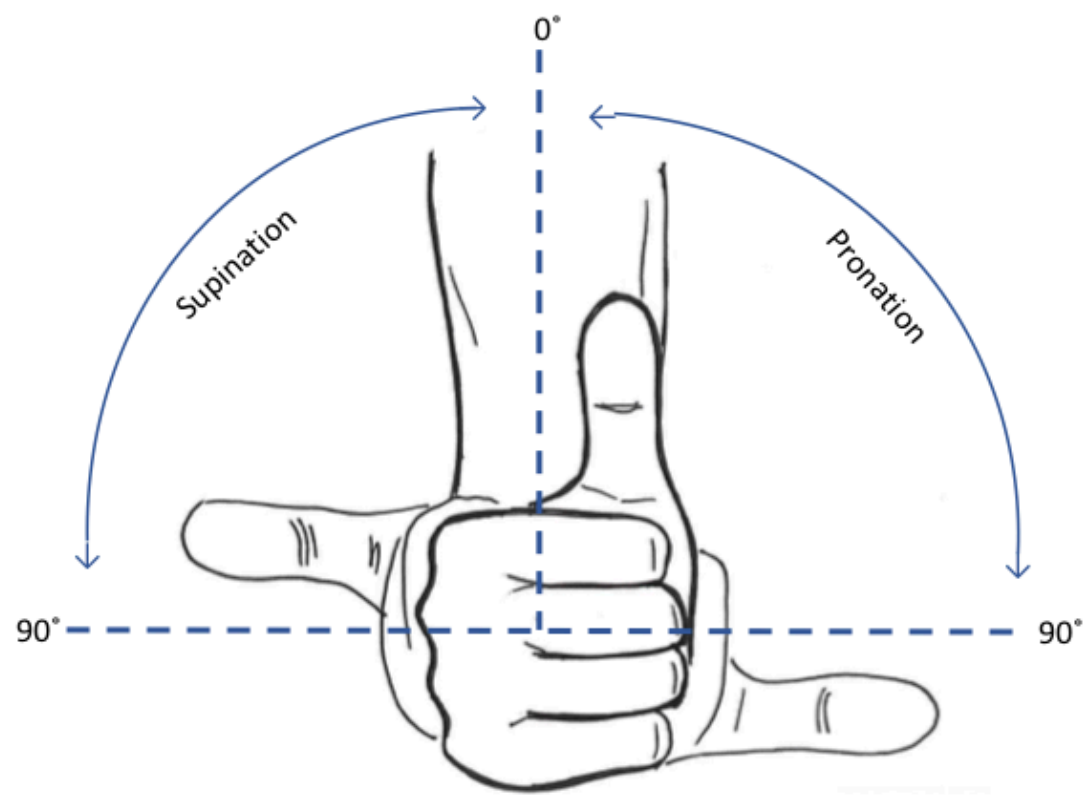

Abbildung 12: Messung der Supination und Pronation des Handgelenks

○ Der Bewegungsradius des Handgelenkes wurde mit Hilfe des „Range of Motion“ (ROM) berechnet. Es wurden Messwerte in Grad von Rlexion und Extension, Radialduktion und Ulnarduktion sowie Supination und Pronation addiert.

Methoden 
- Extension/Flexion in Grund-, Mittel- und Endgelenk des Fingers (Abb. 13 und 14)

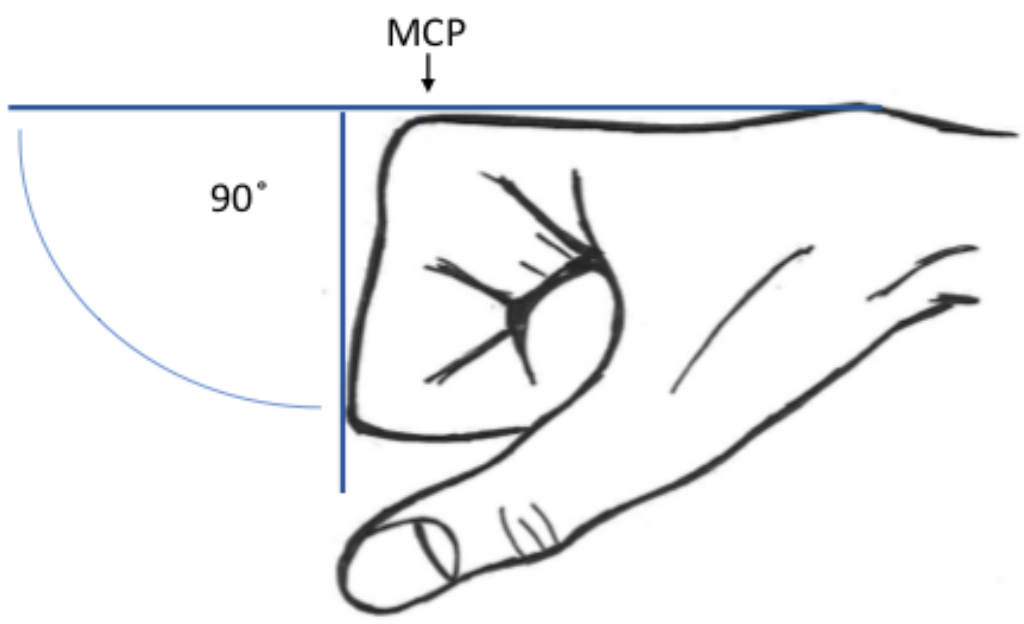

Abbildung 13: Flexion im Grundgelenk des Fingers

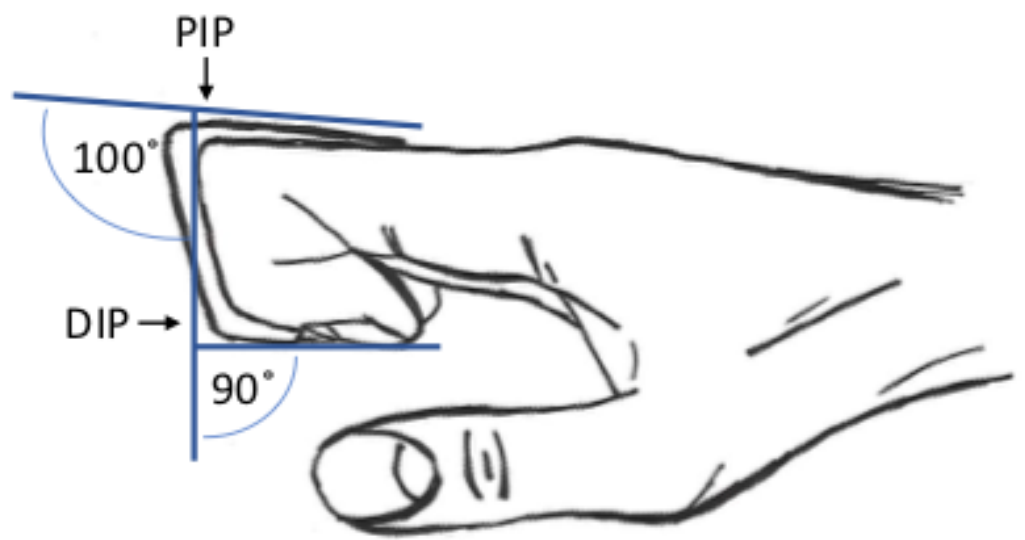

Abbildung 14: Flexion im Mittel- und Endgelenk des Fingers

Methoden

$-46-$ 
- Extension/Flexion im Grund- und Endgelenk des Daumens (Abb. 15)

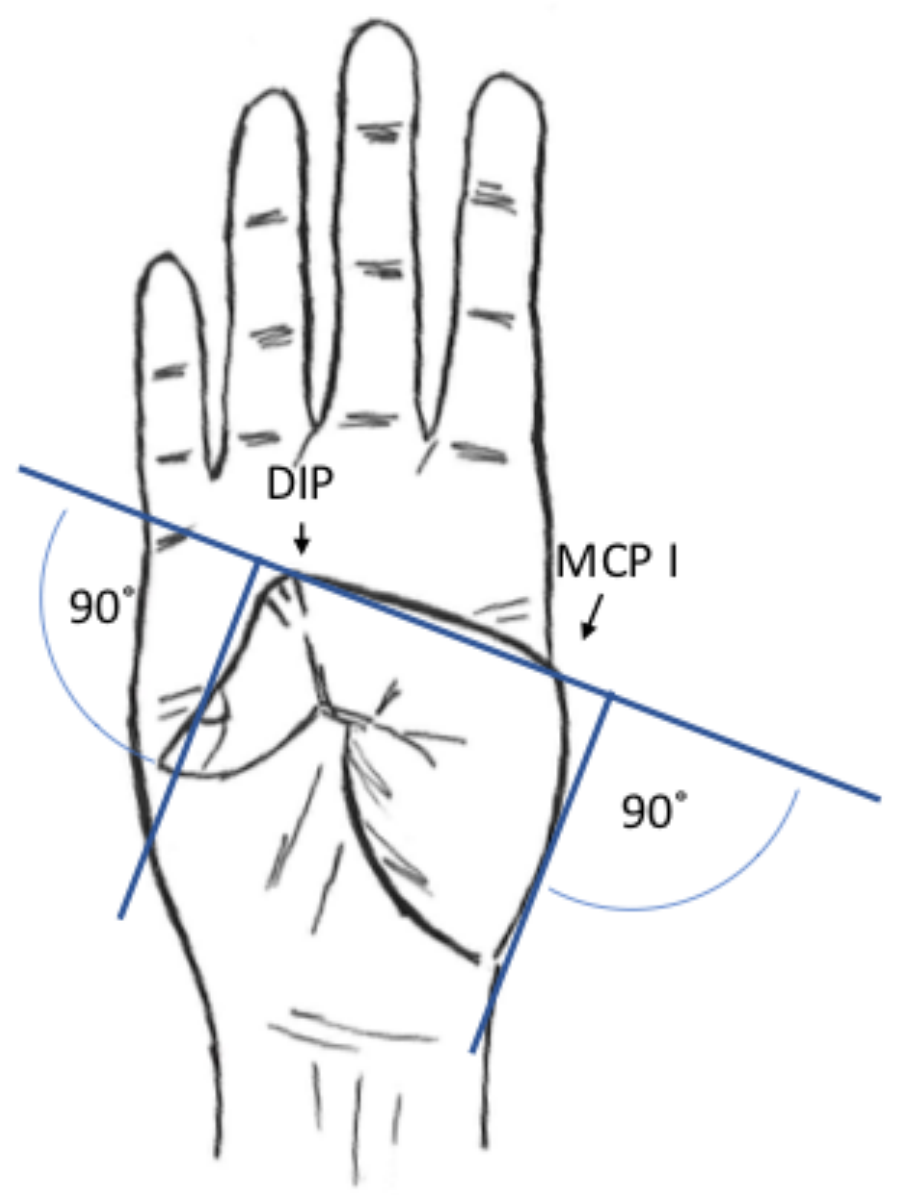

Abbildung 15: Flexion im Grund- und Endgelenk des Daumens

- $\quad$ Total Active Motion (TAM)

Die gesamte Beweglichkeit der Finger sowie das Streckdefizit wurden mit Hilfe der „Total Active Motion“136 (TAM) in Winkelgraden berechnet und mit der Gegenseite verglichen.

$$
\begin{gathered}
\text { TAM }=(\text { MCP-Flexion }+ \text { PIP-Flexion }+ \text { DIP-Flexion })-(\text { MCP-Extensionsdefizit }+ \\
\text { PIP-Extensionsdefizit + DIP-Extensionsdefizit })
\end{gathered}
$$

Bei gleichen Werten an beiden Fingern wurde die TAM als „exzellent“ eingestuft. Wenn die Beweglichkeit der betroffenen Seite mindestens $75 \%$ der Gegenseite betrug, wurde die Beweglichkeit als „gut“" und bei $50 \%$ bis $75 \%$ als „befriedigend“ bewertet. Bei einer Beweglichkeit von weniger als 50 \% verglichen mit der Gegenseite wurde die Beweglichkeit als ,mäßig“‘ bewertet. ${ }^{136}$

Methoden 
- Strickland und Glogovac Kriterien

Strickland und Glogovac ${ }^{137}$ beschreiben 1980 neue Kriterien als eine Modifikation der TAMMethode. Hier wird die gesamte Beweglichkeit sowie das Streckdefizit des betroffenen Fingers nur in PIP- und DIP-Gelenk in Winkelgraden berechnet. Ein Vergleich zur Gegenseite findet nicht statt.

Strickland und Glogovac Berechnung (PIP-Flexion + DIP-Flexion) - (PIP-Extensionsdefizit + DIP-Extensionsdefizit)

Es wird von einem Referenzwert von $175^{\circ}$ in beiden Gelenken ausgegangen. ${ }^{138}$ Die Bewertung erfolgt anhand der Tabelle 5.

\begin{tabular}{|c|c|c|}
\hline Bewertung & Aktive Beweglichkeit in Grad & Normale aktive Beweglichkeit in \% \\
\hline Exzellent & $>150$ & $85-100$ \\
\hline Gut & $125-149$ & $70-84$ \\
\hline Mäßig & $90-124$ & $50-60$ \\
\hline Schlecht & $<90$ & $0-49$ \\
\hline
\end{tabular}

Tabelle 5: Strickland und Glogovac Kriterien

Methoden 
- Kapandji-Index

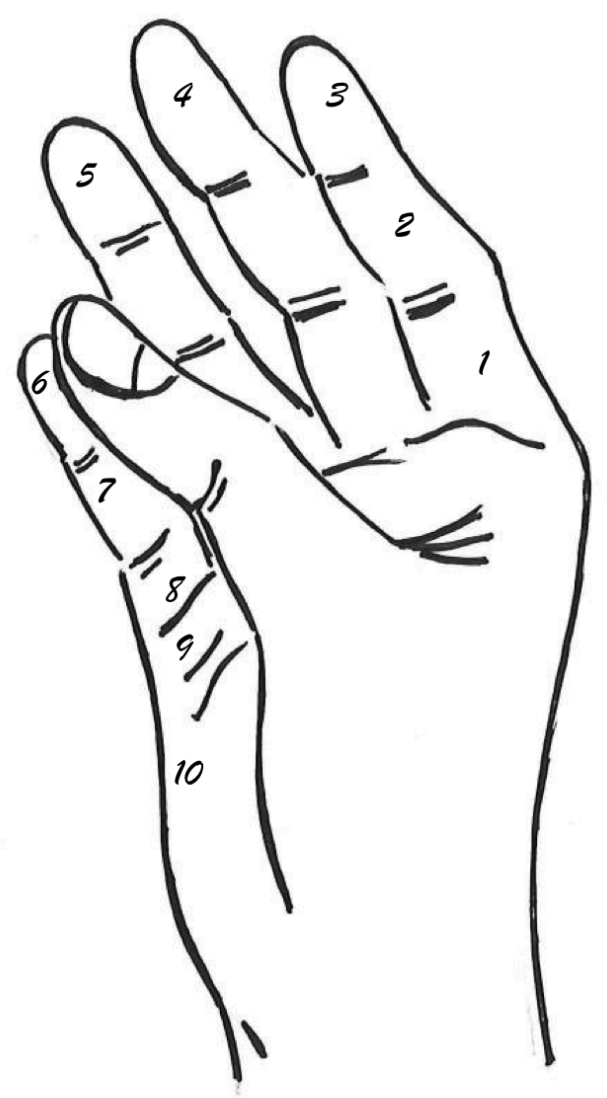

Abbildung 16: Kapandji-Index

Der Kapandji-Index ${ }^{139}$ wurde zur objektiven Messung der Beweglichkeit und Opposition des Daumenstrahlens benutzt (Abb. 16). Der Patient berührt mit der Daumenspitze imaginäre Punkte an der Langfingerinnenfläche derselben Hand. In Abbildung 16 erreicht die Daumenspitze den kleinen Finger und der Kapandji-Index wird hier mit sechs Punkten bewertet. Die Berührung der distalen Hohlbeugefurche entspricht einem Kapandji-Index von 10 und einer maximalen Opposition des Daumens, wohingegen ein Kapandji-Index von eins einer minimalen Daumenopposition entspricht.

Methoden 
- Kraftmessung der Finger mit dem Pinch-Gauge und des Handgelenkes mit dem Jamar-Dynamometer Stufe 2

- Pinch-Gauge ist ein Instrument zur Messung der Fingerkraft im

Schlüsselgriff. Die Standardposition der oberen Extremität wurde wie durch

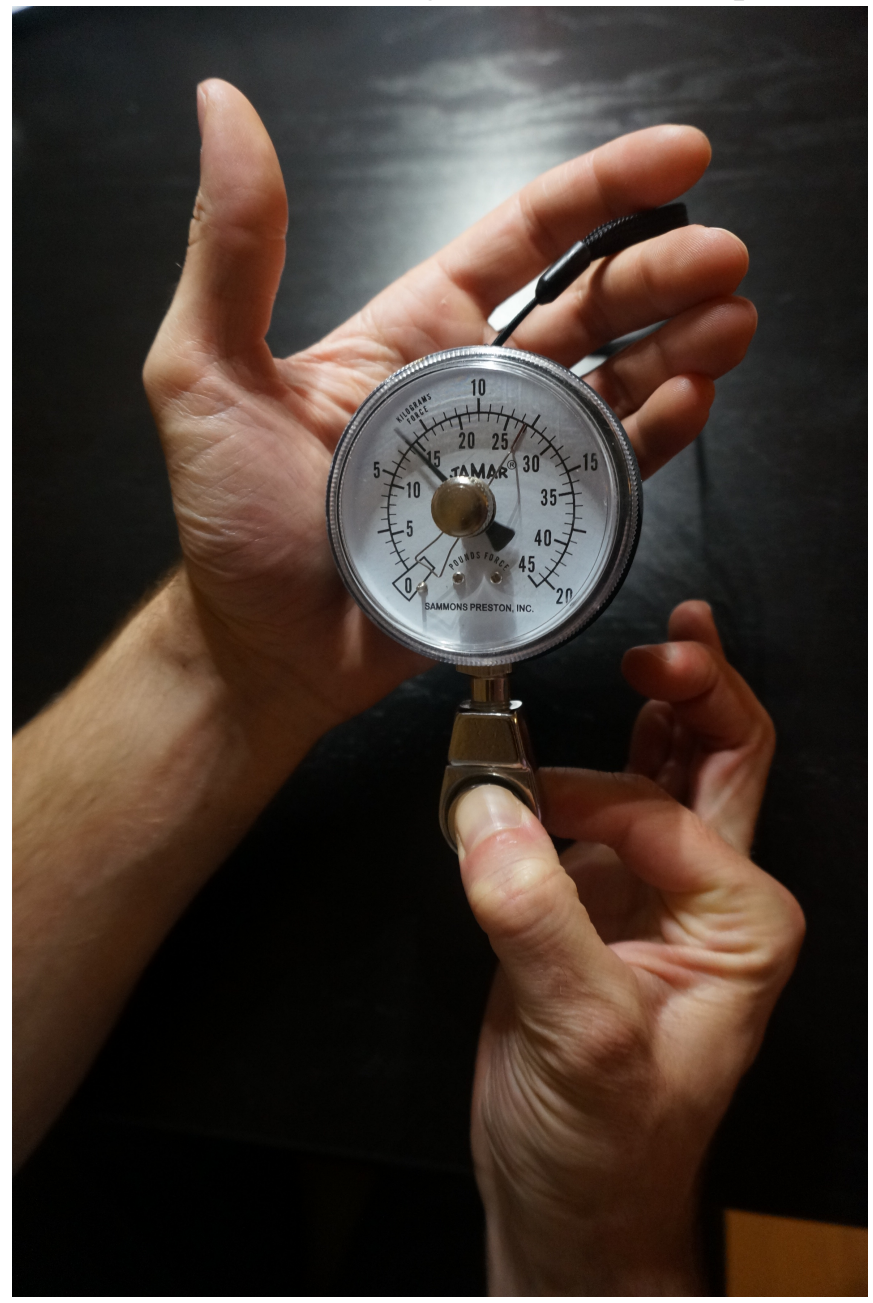

"American Society of Hand

Therapist" (ASHT) beschrieben

angenommen. Die Spitze des

Daumens drückt von oben auf die

Messeinrichtung und die untere

Seite wird nacheinander durch die

vier Fingerspitzen betätigt (Abb.

17). Die Untersuchenden führen

einen Versuch pro Finger gegen den

Daumen mit maximaler Kraft durch.

Die gemessene Werte wurden in

Kilogramm aufgenommen.

Abbildung 17: Kraftmessung zwischen Daumen und Zeigerfinger mittels „Jamar®“ Pinch-Gauge 
- Das Jamar-Dynamometer ist ein zuverlässiges Instrument für die Messung der Kraft und Griffstärke der Handmuskulatur. ${ }^{140}$

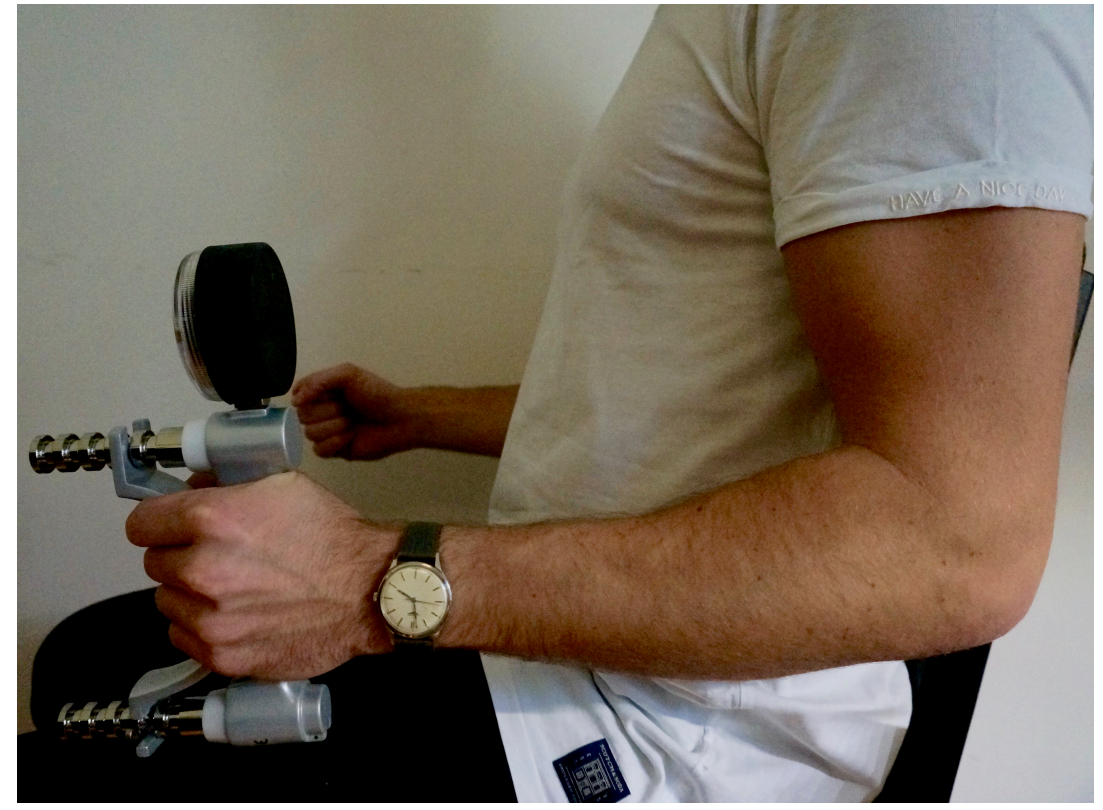

Abbildung 18: Kraftmessung der Handmuskulatur mittels „Jamar@“

Dynamometer Stufe 2

Die Griffeinstellung des Jamar-Dynamometers wurde in Stufe 2 ausgewählt, was eine bestmögliche Messung der Maximalkraft und Bestimmung des Mittelwerts erlaubt. ${ }^{141}$ Die Untersuchenden führten drei Druckversuche mit Maximalkraft und einer Unterbrechung von 60 Sekunden durch. Anhand der Empfehlung der ASHT wurde die Messung in Standardposition bei sitzender Haltung mit adduziertem Oberarm, 90 flektiertem Ellenbogen, Unterarm sowie Handgelenk in Neutral-Null-Stellung, durchgeführt (Abb. 18). ${ }^{142}$ Die resultierenden Messwerte wurden als Mittel- sowie Maximalwert in Kilogramm dokumentiert. 
- Neurologische Untersuchung mit Erfassung von Sensibilitätsdefiziten im Vergleich zur Gegenseite durch Messung der Zwei-Punkte-Diskrimination und dem SemmesWeinstein Monofilament-Test

○ Die statische Zwei-Punkte-Diskrimination (2-PD) beschreibt die Fähigkeit der Hautoberfläche ein oder zwei mit gleichem Druck aufgesetzte Stimuli

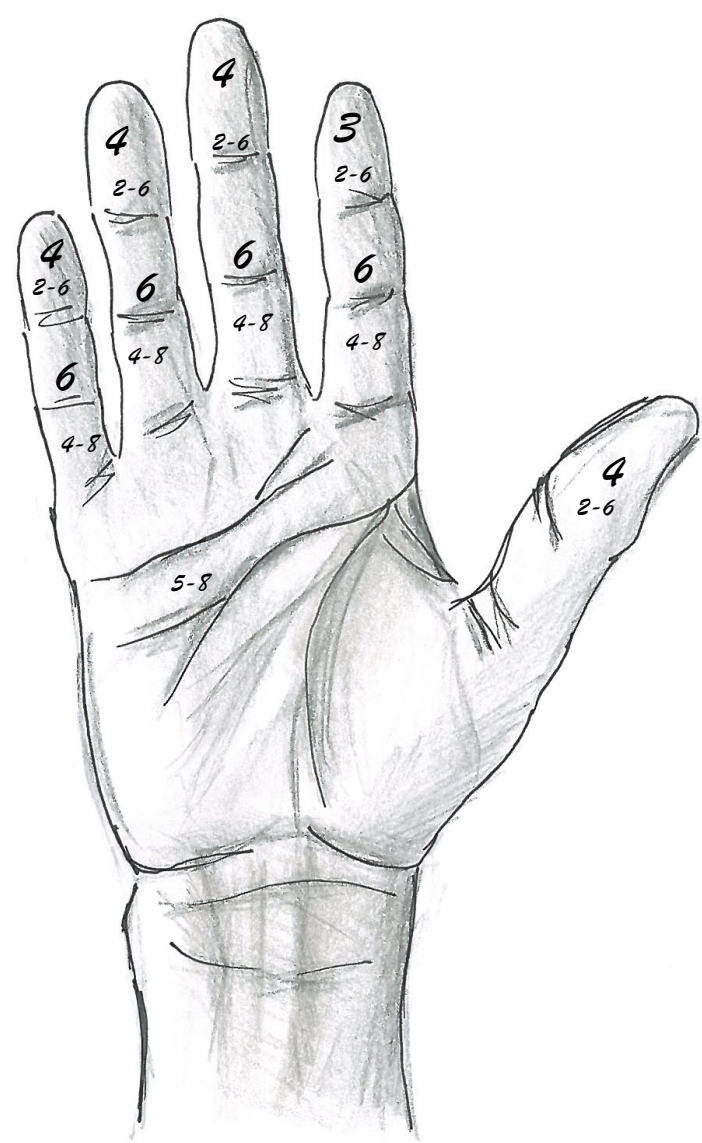

Abbildung 19: Zwei-Punkte-Diskrimination in mm nach Weber wahrzunehmen und voneinander $\mathrm{zu}$ unterscheiden. ${ }^{143-145}$ Mit Hilfe der ZweiPunkte-Diskriminatorscheibe wird die Oberflächensensibilität am Fingerspitzenbereich der betroffenen sowie der gesunden Finger nachuntersucht. Die Spitzen des Diskriminators werden statisch für mindestens drei Sekunden auf die Haut der Fingerkuppe radial und ulnar aufgesetzt. Unter Angaben des Untersuchten und bei visuellem Ausschluss werden größere bzw. kleinere Spitzenabstände des Testgeräts an der Haut gewechselt. Die physiologische 2PD liegt am Fingerkuppenbereich zwischen 2 bis $6 \mathrm{~mm}$ (Abb. 19). ${ }^{146-148}$ 
O Semmes-Weinstein Monofilament-Test ${ }^{149}$ (SWMT) wurde 1960 durch die Psychologen Semmes und Weinstein zur Beurteilung von Sensibilitätsdefiziten an der Hand bei Patienten mit Hirnverletzung entwickelt. Der leichte Druck wird mit einem aus Nylon bestehenden Faden senkrecht an der radialen und ulnaren Seite der betroffenen Fingerspitze sowie am Finger der Gegenseite ausgeübt (Abb. 20). Die fünf verschiedenen Monofilamente werden in aufsteigender Reihenfolge getestet. Es wird mit dem der normalen Hautsensibilität entsprechenden Monofilament 2,83N begonnen. Die Monofilamente 1,65N bis $4,31 \mathrm{~N}$ werden drei Mal an der gleichen Stelle radial und ulnar an der Fingerkuppe

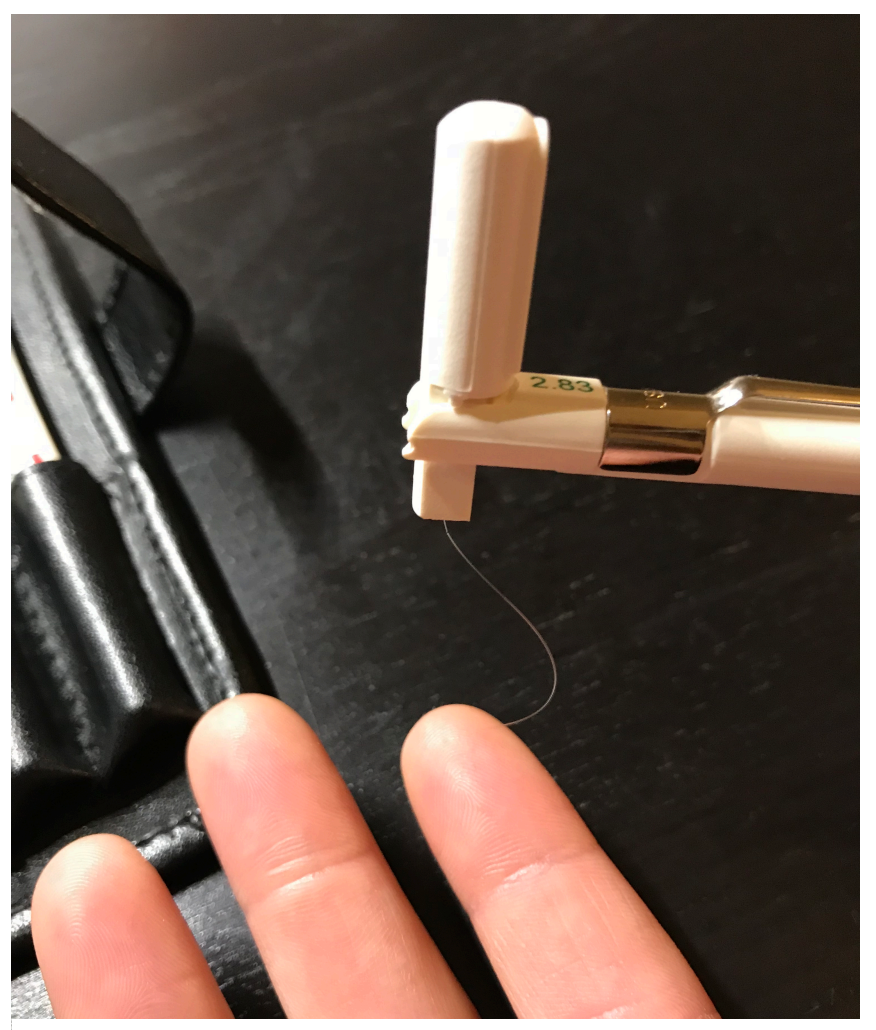

Abbildung 20: Sensibilität an der Fingerkuppe mittels Semmes-Weinstein Monofilament-Test für 1,5 s mit der Pause von 1,5 $\mathrm{s}$ appliziert, die höhere Monofilamentenstufen dagegen nur ein Mal. Die Messung erfolgt unter dem Ausschluss visueller Kontrolle und der Untersuchte gibt das Empfinden der Berührung an. Monofilament Beschreibung:

- $1,65-2,83 \mathrm{~N}=$ normale Sensibilität

- $\quad 3,22-3,61 \mathrm{~N}=$ eingeschränkte Berührungsempfindung

- $\quad 3,84-4,31 \mathrm{~N}=$ verminderte, verzögerte Schutzsensibilität

- $\quad 4,56-6,65 \mathrm{~N}=$ Verlust der

Schutzsensibilität

- $\quad>6,65 \mathrm{~N}=$ keine Sensibilität vorhanden 
- Erfassung von Nagelwachstumsstörungen und insbesondere der Ausbildung eines Krallennagels mit Hilfe der Lim-Klassifikation ${ }^{30}$ (Abb. 21) inklusive Fotodokumentation sowie eine Messung der Nagellänge beidseits

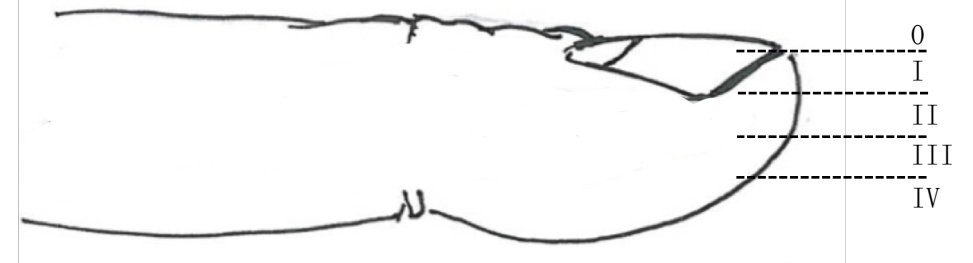

Abbildung 21: Lim-Klassifikation des Krallennagels

- Fotodokumentation der Finger im Vergleich zur gesunden Seite

\section{Statistische Auswertung}

Die erhobenen Daten wurden statistisch mittels Microsoft Excel und des biometrischen Statistikprogramms SPSS, Version 25.0 für Mac ausgewertet.

Der Mittelwert (MW), die Standardabweichung (SD) und die Spannweite (R) wurden zur Beschreibung der metrischen Parameter berechnet.

Der Vergleich der Mittelwerte von abhängigen Stichproben, wie beispielweise Vergleich der mittleren Kraft, mit dem Jamar-Dynamometer, des betroffenen Handgelenkes und der Gegenseite, erfolgte mit dem t-Test für paarweise Vergleiche.

Der Vergleich der Mittelwerte von unabhängigen Stichproben, wie beispielweise Vergleich der Dauer der Arbeitsunfähigkeit zwischen durch die Berufsgenossenschaft versicherten Patienten und nicht durch die Berufsgenossenschaft Versicherten, erfolgte mit dem t-Test für unabhängige Stichproben.

Die statistische Auswertung der Score erfolgte mit Hilfe des Wilcoxon-Mann-Whitney-UTestes.

Für die gesamte Studie wurde eine Irrtumswahrscheinlichkeit unter 5\% $(\mathrm{p}<0,05)$ als statistisch signifikant und unter $0,1 \%(\mathrm{p}<0,001)$ als statistisch hoch signifikant angenommen.

Methoden 
Der Zusammenhang von zwei metrischen Parametern, wie beispielweise die Korrelation zwischen den Schmerzen in Ruhe und unter Belastung, wurde mittels des Korrelationskoeffizienten nach Pearson ausgewertet.

Die Berechnung des Zusammenhangs zwischen Ereignissen, wie unter anderem den Ort des Unfalls und der Art der Fingerkuppenverletzung, erfolgte mittels der Kreuztabellen und dem Chi-Quadrant-Test nach Pearson.

Ein Korrelationskoeffizient über 0,7 wurde als deutlicher Zusammenhang angenommen.

Methoden 


\section{Ergebnisse}

7.1 Auswertung der Patientenakten von 53 Patienten mit 57 Fingerkuppenverletzungen

\subsubsection{Alter und Geschlechtsverteilung}

Zum Zeitpunkt der Operation betrug das mittlere Alter der Patienten 38,4 $\pm 15,1$ Jahre. Der jüngste operierte Patient war im Alter von 11, der älteste war 73 Jahre alt. Es handelte sich um 47 Männer (88,7 \%) und um 6 Frauen (11,3\%). Die Abbildung 22 zeigt die Anzahl der aufgenommenen Patienten in den verschiedenen Altersgruppen.

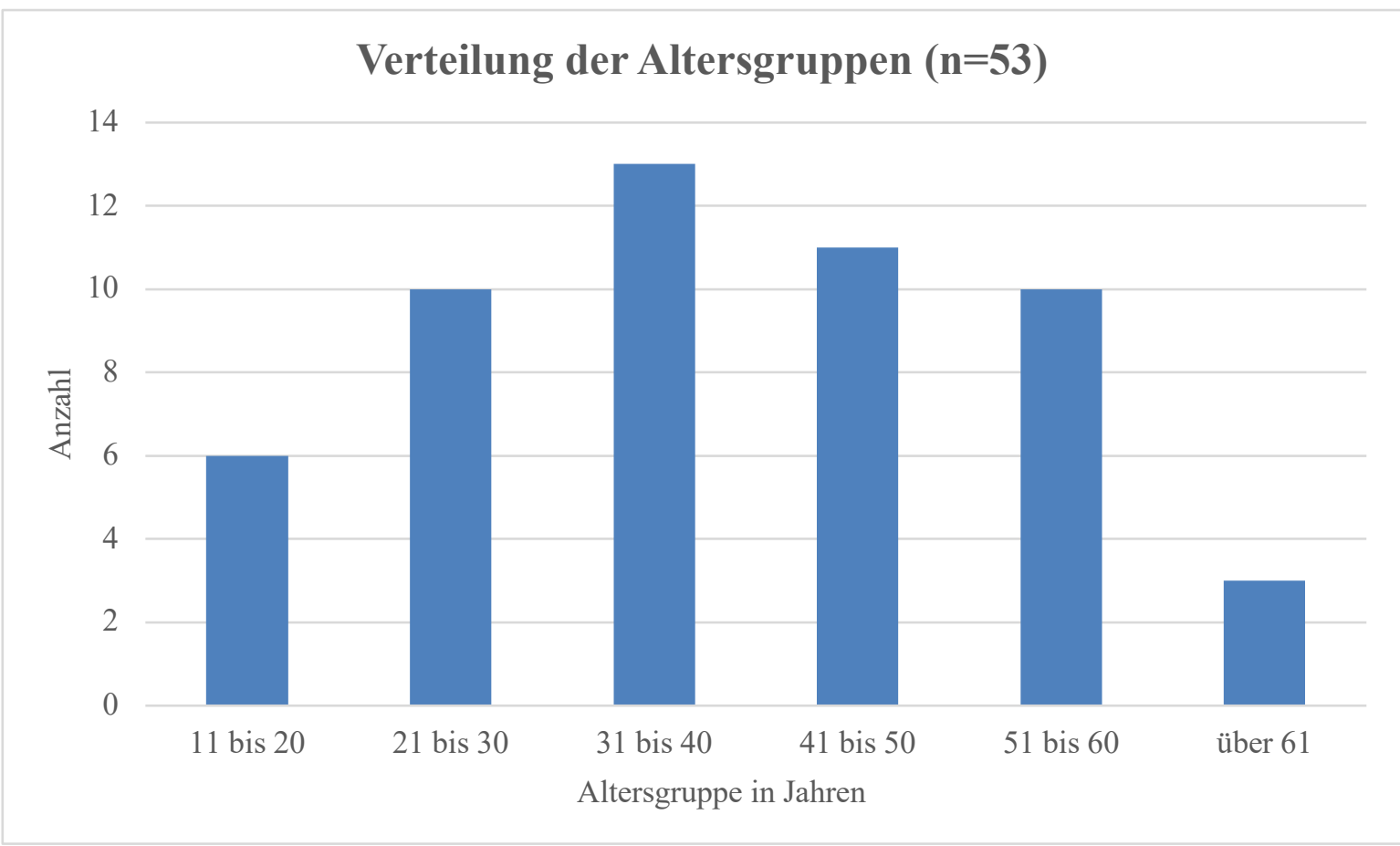

Abbildung 22: Anzahl der in die Studie aufgenommenen Patienten der verschiedenen Altersgruppen

\subsubsection{Ort des Unfalls und Art der Fingerkuppenverletzungen}

In 39 Fällen (73,6 \%) geschah die Fingerkuppenverletzungen in Folge eines Arbeitsunfalles und in 14 Fällen (26,4 \%) als häuslicher Unfall.

Nach der Allen-Klassifikation der Fingerkuppenverletzungen waren 49 in Zone III (86 \%) und 8 in Zone IV (14\%) eingeteilt. 


\subsubsection{Dauer des stationären Aufenthalts}

Die mittlere Aufenthaltsdauer im Krankenhaus betrug 5,6 4 4,7 Tage. Ein Patient wurde lediglich ambulant versorgt, der längste stationäre Aufenthalt lag bei 14 Tagen. Hier suggerieren andere Begleitverletzungen eine höhere Liegedauer.

\subsubsection{Lokalisation der Fingerkuppenverletzung}

Wie Abbildung 23 veranschaulicht, traten am häufigsten Verletzungen an Mittel-, Zeige- und Ringfinger auf.

Von 53 Patienten erlitten 18 Patienten (31,6 \%) zeitgleich eine Verletzung an ein bis drei weiteren benachbarten Fingern, welche jedoch nicht mit neurovaskulären Insel-Lappen gedeckt wurden. Die häufigste Kombination der Verletzung war in 8 Fällen der Zeige- und Mittelfinger (44,4 \%) sowie in 6 Fällen der Mittel- und Ringfinger (33,3 \%). 


\section{Häufigkeit aller verletzten Fingerkuppen $(\mathbf{n = 8 1})$}

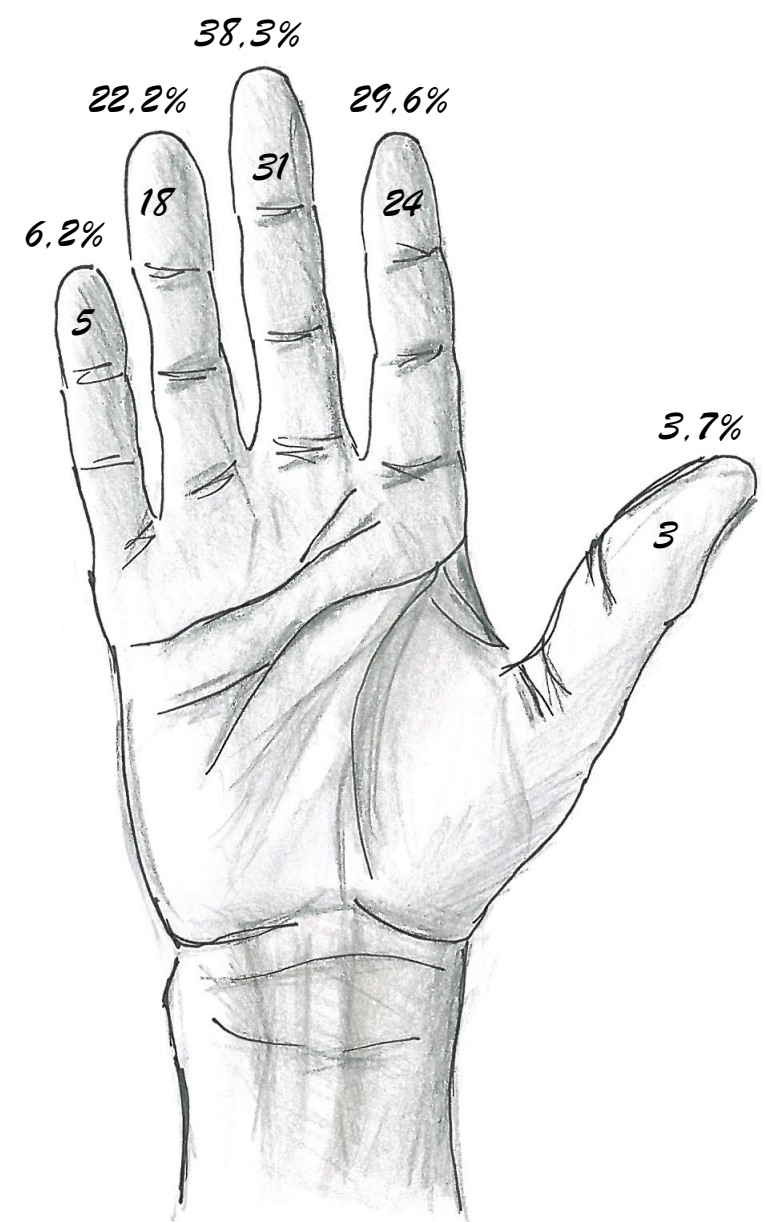

Abbildung 23: Häufigkeit aller verletzten Fingerkuppen

Von 81 insgesamt verletzten Fingern wurden 57 (70,4 \%) durch neurovaskuläre Insel-Lappen versorgt. Wie in Tabelle 6 dargestellt, ergibt sich eine geringfügig verstärkte Häufung rechts zwischen allen verletzten und durch neurovaskulären Insel-Lappen versorgten Fingerkuppen. 


\begin{tabular}{|c|c|c|c|c|c|c|}
\hline \multirow{2}{*}{$\begin{array}{c}\text { Betroffene } \\
\text { Seite }\end{array}$} & \multicolumn{2}{|c|}{ Hand } & \multicolumn{2}{c|}{$\begin{array}{c}\text { Verletzte } \\
\text { Fingerkuppen }\end{array}$} & $\begin{array}{c}\text { Durch neurovaskuläre } \\
\text { Insel-Lappen versorgte } \\
\text { Fingerkuppen }\end{array}$ \\
\cline { 2 - 7 } & n & $\%$ & n & \% & n & $\%$ \\
\hline Rechts & 29 & 54,7 & 44 & 54,3 & 31 & 54,4 \\
\hline Links & 24 & 45,3 & 37 & 45,7 & 26 & 45,6 \\
\hline$\Sigma$ & 53 & 100 & 81 & 100 & 57 & 100 \\
\hline
\end{tabular}

Tabelle 6: Anzahl der verletzten und durch neurovaskuläre Insel-Lappen versorgten Fingerkuppen.

Die Abbildung 24 zeigt die Anzahl der durch neurovaskuläre Insel-Lappen versorgten Fingerkuppen. Am häufigsten wurden proportional zu den Verletzungen die Zeige-, Mittel und Ringfinger rekonstruiert. 
Häufigkeit der rekonstruierten Fingerkuppen $(n=57)$

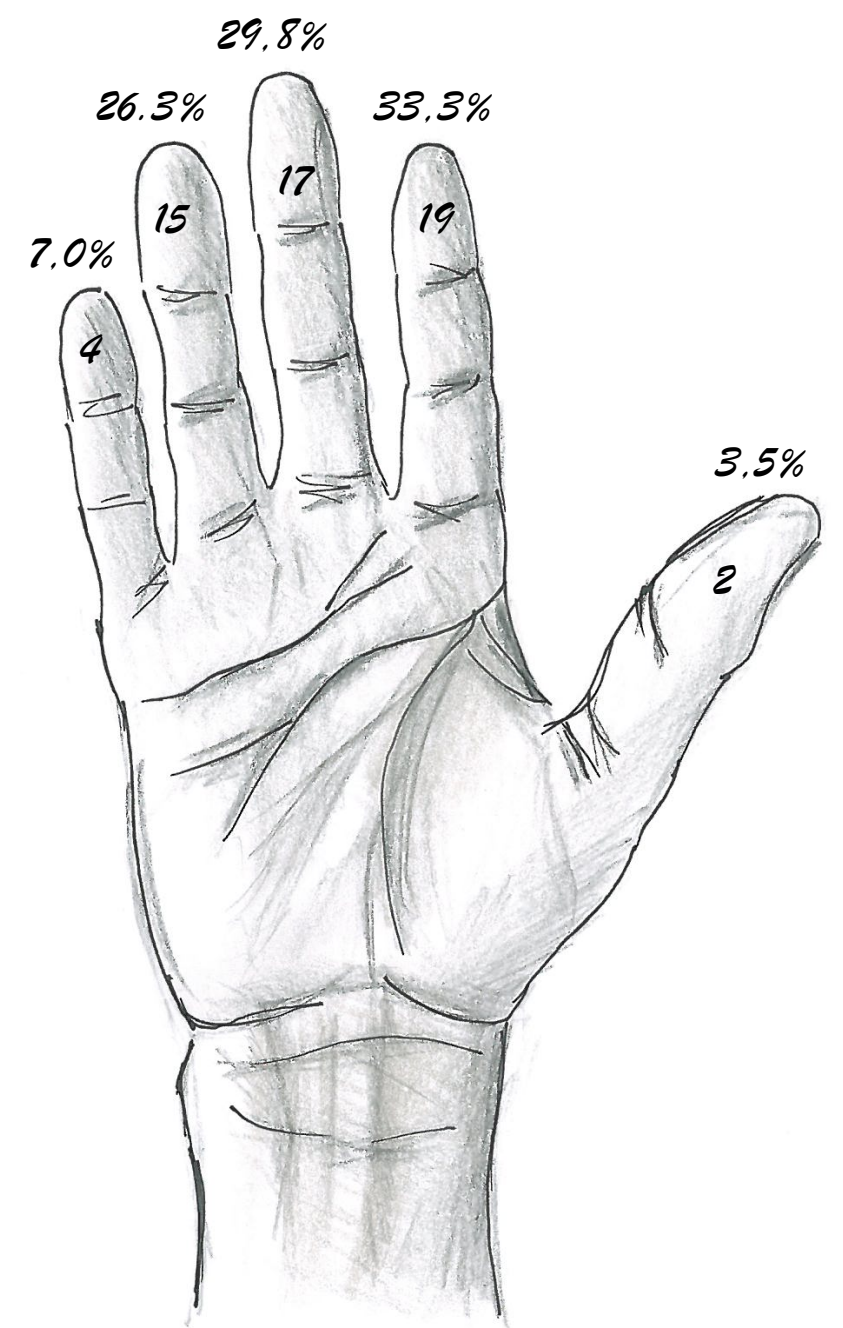

Abbildung 24: Häufigkeit der verletzten Fingerkuppen, welche durch neurovaskuläre Insel-Lappenplastik versorgt wurden

Ergebnisse

$-60-$ 


\subsubsection{Hebestelle von neurovaskulären Insel-Lappen}

Die Defektdeckung wurde in 31 Fällen von radial $(54,4 \%)$, in 23 von ulnar $(40,3 \%)$ und in weiteren drei Fällen $(5,3 \%)$ durch einen beidseitig verlaufenden neurovaskulären InselLappen durchgeführt.

Die Tabelle 7 zeigt die Häufigkeit der ausgewählten Hebestelle von neurovaskulären InselLappen auf die rekonstruierten Fingerkuppen. Wie Abbildung 25 veranschaulicht, wurden am häufigsten radialseitige Insel-Lappen am Zeigerfinger angewandt.

\begin{tabular}{|c|c|c|c|c|c|c|}
\hline Lappen & \multicolumn{2}{|c|}{ radial } & \multicolumn{2}{c|}{ ulnar } & \multicolumn{2}{c|}{ beidseitig } \\
\hline Finger & $\mathbf{n}$ & $\%$ & $\mathbf{n}$ & $\%$ & $\mathbf{n}$ & $\%$ \\
\hline I & 1 & 3,2 & 1 & 4,3 & - & - \\
\hline II & 13 & 41,9 & 6 & 26,0 & - & - \\
\hline III & 8 & 25,8 & 8 & 34,7 & 1 & 33,3 \\
\hline IV & 8 & 25,8 & 7 & 30,4 & - & - \\
\hline V & 1 & 3 & 1 & 4,3 & 2 & 66,6 \\
\hline $\boldsymbol{\Sigma}$ & 31 & 100 & 23 & 100 & 3 & 100 \\
\hline
\end{tabular}

Tabelle 7: Häufigkeit der ausgewählten Hebestelle der neurovaskulären Insel-Lappen

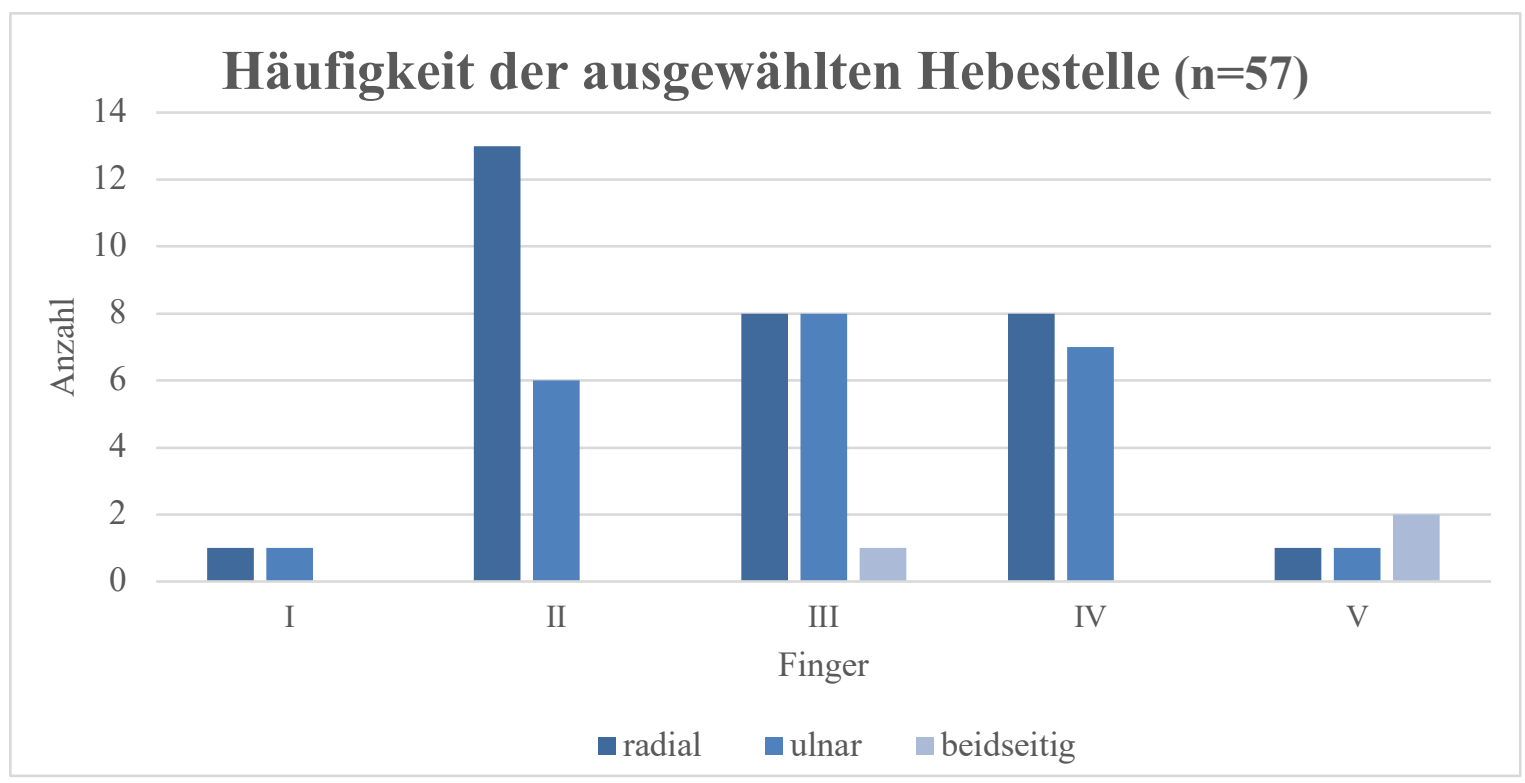

Abbildung 25: Häufigkeit der ausgewählten Hebestelle der neurovaskulären Insel-Lappen Ergebnisse $-61-$ 


\subsubsection{Frühkomplikationen und Notwendigkeit von Folgeoperationen}

Von 53 Patienten bestand bei insgesamt 7 (24,5\%) die Notwendigkeit einer erneuten Operation. Bei einem Patienten (1,9\%) trat unter Einnahme von Acetylsalicylsäure $100 \mathrm{mg}$ eine Wunddehiszenz infolge einer Nachblutung auf, sodass am 4. Tag eine Revision und Blutstillung durchgeführt wurde. Ein Patient erlitt eine Woche nach der initialen Operation eine Infektion sowie Teilnekrose des Endgliedes mit der Notwendigkeit einer Nachamputation unter Erhalt des neurovaskulären Insel-Lappens. Ein elfjähriges Kind wurde 2,4 Monate nach der initialen Operation wegen Entstehung einer Narbenkontraktur am verletzten Finger erneut operiert und der Narbenstrang nach Exzision mit VY-Plastiken aufgelöst.

Bei vier Patienten wurde eine geplante zweite Operation zur Kunsthautentfernung mit Sekundärnaht oder Vollhautdeckung durchgeführt.

Eine Osteosynthese war bei keiner Operation erforderlich. Bei den nachuntersuchten Patienten wurden keine Nachoperationen in anderen Krankenhäusern durchgeführt. Die Langzeitkomplikationen, die konservativ behandelt wurden (z.B. Kälteempfindlichkeit oder Sensibilitätsstörungen) werden im Kapitel 7.2.7 beschrieben.

\subsection{Patientenbefragung von 28 Patienten mit 29 Fingerkuppenverletzungen}

\subsubsection{Epidemiologische Daten}

Zum Zeitpunkt der Nachuntersuchung lag das mittlere Alter der 28 Patienten bei 52,4 $\pm 15,0$ Jahren (Abb. 26). Die Spannweite des Alters reichte von 22 bis 79 Jahren. Es handelte sich um 24 Männer (85,7 \%) und 4 Frauen (14,3\%). 


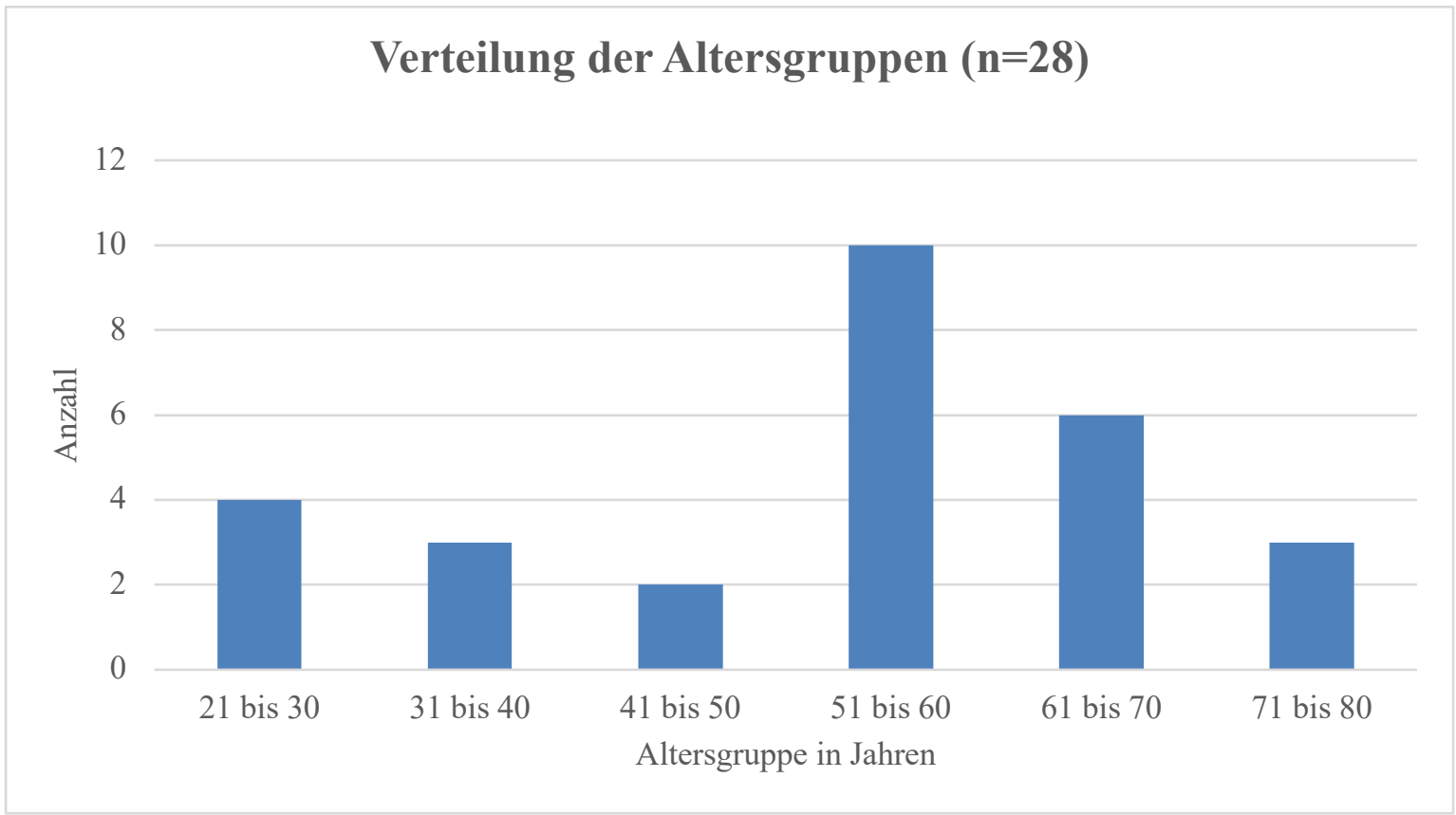

Abbildung 26: Anzahl nachuntersuchte Patienten in den verschiedenen Altersgruppen

Die Händigkeit der 28 Patienten wurde über die Schreibhand definiert. Als dominierende Hand gaben 25 Patienten die rechte Hand (89,3\%) und 3 die linke Hand (10,7\%) an. Bei 14 Rechtshändern (56 \%) war die dominierende Hand die verletzte.

\subsubsection{Vorerkrankungen, Medikamente und Raucheranamnese}

Von 28 Patienten hatten $23(82,1 \%)$ keine für den weiteren Verlauf relevanten Vorerkrankungen oder nahmen Medikamente ein.

Ein Patient hatte Spondylolistesis, zwei Knie- bzw. Hüftarthrose und einer Diabetes mellitus Typ II. In einem Fall trat unter regelmäßiger Einnahme von Acetylsalicylsäure 100 mg, wie im Kapitel 7.1.6 beschrieben, eine Nachblutung auf.

Zum Zeitpunkt der Operation haben 9 Patienten (32,1 \%) täglich ca. 18 Zigaretten geraucht.

\subsubsection{Dauer der Nachuntersuchung, Arbeitsunfähigkeit und endgültigen Heilung}

Der Mittelwert der Nachuntersuchungsdauer betrug 105,0 \pm 42,3 Monate mit einer Spannweite von 42 bis 169 Monaten. 
Der mittlere Wert der Arbeitsunfähigkeitsdauer bei allen 27 arbeitsfähigen Patienten lag bei 6,1 \pm 3,3 Wochen mit einer Spannweite von 1 bis 12 Wochen. Ein Patient befand sich zum Zeitpunkt der Operation bereits in Rente.

Wie Tabelle 8 zeigt, waren Patienten, die durch die Berufsgenossenschaften versichert waren, im Mittel ca. 2,5 Wochen länger krankgeschrieben.

Der Vergleich der Arbeitsunfähigkeitsdauer beider Gruppen wurde mittels t-Test bei unabhängigen Stichproben mit ungleichen Varianzen durchgeführt. Der Unterschied konnte als statistisch signifikant $(\mathrm{p}=0,05)$ nachgewiesen werden.

\begin{tabular}{|c|c|c|}
\hline \multirow{2}{*}{ Ort des Unfalls } & \multicolumn{2}{|c|}{ Arbeitsunfähigkeit (Wochen) } \\
\cline { 2 - 3 } & MW \pm SD & R \\
\hline Arbeitsunfall (n=18) & $6,9 \pm 3,4$ & $1-12$ \\
\hline Häuslicher Unfall (n=9) & $4,4 \pm 2,5$ & $1-10$ \\
\hline $\boldsymbol{\Sigma}(\mathbf{n}=\mathbf{2 7})$ & $6,1 \pm 3,3$ & $1-12$ \\
\hline
\end{tabular}

Tabelle 8: Dauer der Arbeitsunfähigkeit beim Arbeitsunfall und häuslichem Unfall

Von insgesamt 18 Patienten übten 16 zum Zeitpunkt des Arbeitsunfalls einen Handwerksberuf aus, dabei war der Beruf des Montagearbeiters bzw. Mechanikers mit jeweils drei Patienten am häufigsten vertreten. Von 9 häuslichen Unfällen waren die Berufsgruppen Kaufmann bzw. Schüler mit jeweils drei und zwei Patienten am häufigsten betroffen.

Die Zeit bis zur endgültigen Heilung der 28 befragten Patienten betrug 14,6 \pm 9,6 Wochen mit einer Spannweite von 4 bis 48 Wochen (retrospektive Selbsteinschätzung des Patienten zum Zeitpunkt der Nachuntersuchung).

Die Tabelle 9 vergleicht die Heilungsdauer beim Arbeits- und häuslichen Unfall. Die Heilungsdauer der durch die Berufsgenossenschaft versicherten Patienten war im Mittel ca. 4 Wochen kürzer als die Heilung der nicht durch die Berufsgenossenschaften versicherten Patienten. 
Der Unterschied zwischen beiden Gruppen wurde mittels t-Test bei unabhängigen Stichproben mit ungleichen Varianzen konnte nicht als statistisch signifikant $(p=0,41)$ nachgewiesen werden.

\begin{tabular}{|c|c|c|}
\hline \multirow{2}{*}{ Ort des Unfalls } & \multicolumn{2}{|c|}{ Heilungsdauer (Wochen) } \\
\cline { 2 - 3 } & MW \pm SD & $\mathbf{R}$ \\
\hline Arbeitsunfall (n=19) & $13,1 \pm 5,5$ & $6-24$ \\
\hline Häuslicher Unfall (n=9) & $17,2 \pm 13,8$ & $4-48$ \\
\hline $\boldsymbol{\Sigma}(\mathbf{n}=\mathbf{2 8})$ & $14,6 \pm 9,6$ & $4-48$ \\
\hline
\end{tabular}

Tabelle 9: Endgültige Heilungsdauer beim Arbeitsunfall und häuslichem Unfall

\subsubsection{Physio- und Ergotherapie}

Insgesamt bei 16 Patienten (57,1 \%) wurde eine Physio- bzw. Ergotherapie zur Nachbehandlung in Anspruch genommen. Von 18 durch die Berufsgenossenschaft versicherten Patienten haben 10 (55,5\%) und von 10 nicht durch die Berufsgenossenschaft Versicherten haben 6 Patienten (60 \%) eine Physio- bzw. Ergotherapie bekommen.

\begin{tabular}{|c|c|c|}
\hline \multirow{2}{*}{ Ort des Unfalls } & \multicolumn{2}{|c|}{ Sitzungen (n) } \\
\cline { 2 - 3 } & MW \pm SD & R \\
\hline Arbeitsunfall (n=10) & $14,9 \pm 16,2$ & $2-60$ \\
\hline Häuslicher Unfall (n=6) & $16 \pm 11,4$ & $7-40$ \\
\hline$\Sigma(\mathbf{n}=\mathbf{1 6})$ & $15,3 \pm 14,6$ & $2-60$ \\
\hline
\end{tabular}

Tabelle 10: Anzahl der Physio- und Ergotherapie

Wie aus Tabelle 10 hervorgeht, war die mittlere Anzahl der Sitzungen von Physio- bzw. Ergotherapie in beiden Patientengruppen fast identisch. Mittels eines t-Tests bei unabhängigen Stichproben wurde kein Nachweis einer statistisch signifikanten Differenz (p $=0,88)$ festgestellt. 


\subsubsection{Schmerzempfindlichkeit in Ruhe und unter Belastung}

Von 28 nachuntersuchten Patienten gaben 21 (75\%) in Ruhe und 15 (53,6 \%) unter Belastung keine Schmerzen an.

Wie in Tabelle 11 dargestellt, lag der Mittelwert der Schmerzempfindlichkeit sowohl in Ruhe als auch unter Belastung im unteren Bereich der numerischen Rating-Skala mit einer Verstärkung der Schmerzen unter Belastung um etwa die Hälfte. Ein Patient bewertete die Schmerzen der Fingerkuppe unter Belastung als stark und gab die Zahl 9 der NRS an.

\begin{tabular}{|c|c|c|}
\hline \multirow{2}{*}{ Schmerzen } & \multicolumn{2}{|c|}{ NRS } \\
\cline { 2 - 3 } & MW \pm SD & R \\
\hline In Ruhe & $1,2 \pm 2,1$ & $0-7$ \\
\hline Unter Belastung & $2,4 \pm 2,9$ & $0-9$ \\
\hline
\end{tabular}

Tabelle 11: Werte der Schmerzempfindlichkeit in Ruhe und unter Belastung anhand der numerischen Rating-Skala

\subsubsection{Subjektive bzw. objektive Bewertung von Schweißbildung und Temperatur}

Schweißbildung der 29 mit neurovaskulärem Insel-Lappen gedeckten Fingerkuppen wurde durch die nachuntersuchten Patienten subjektiv in 26 Fällen (89,7 \%) als normal, in zwei als vermindert und in einem als erhöht angegeben. Dagegen konnte Schweißbildung objektiv in 28 Fällen (96,6 \%) als normal und in einem als vermindert beschrieben werden.

Die Temperatur der rekonstruierten Fingerkuppe empfanden 20 Patienten (69 \%) als kühl und $9(31 \%)$ als normal.

\subsubsection{Langzeitkomplikationen und Probleme an der Entnahmestelle}

Es konnten 29 Fingerkuppen für die Erfassung von Neurombeschwerden, Schwellugen, Kälteempfindlichkeit, Vorhandensein von Hoffmann-Tinel-Zeichen sowie Druckschmerz untersucht und bewertet werden.

Keiner der nachuntersuchten Patienten wies Neurombeschwerden auf. Bei einem Patienten war seit dem Unfall eine leichte Schwellung im Bereich des Metakarpophalangealgelenks entstanden. Kälteempfindlichkeit gaben 14 Patienten (48,3 \%) an, 7 (24,1 \%) objektives 
Hoffmann-Tinel-Zeichen und 5 (17,2 \%) Druckschmerz im Bereich der verletzten Fingerkuppe.

Von 28 nachuntersuchten Patienten gaben 20 (71,4 \%) keine subjektiv empfundenen Probleme an der Entnahmestelle an. Drei Patienten (10,7 \%) klagten über Missempfindungen, vier (14,3\%) über subjektives Hoffmann-Tinel-Zeichen und einer (3,6 $\%)$ über Schmerzen.

\subsubsection{Subjektive Patientenzufriedenheit und DASH-Score}

Der Mittelwert der Patientenzufriedenheit lag bei 8,7 $\pm 1,4$ mit einer Spannweite von 5 bis $10(0=$ maximal unzufrieden, $10=$ maximal zufrieden $)$. Alle befragten Patienten würden sich wieder operieren lassen und waren mit dem Operationsergebnis zufrieden.

\begin{tabular}{|c|c|c|c|}
\hline \multirow{2}{*}{ DASH Score } & \multicolumn{3}{|c|}{ Patienten } \\
\cline { 2 - 4 } & $\mathbf{n}$ & MW \pm SD & R \\
\hline ADL & 28 & $16,0 \pm 17,9$ & $0-66,7$ \\
\hline Arbeit & 20 & $17,2 \pm 21,1$ & $0-68,8$ \\
\hline Sport & 19 & $15,5 \pm 21,7$ & $0-68,8$ \\
\hline
\end{tabular}

Tabelle 12: Der Mittelwert des DASH-Score für alle befragten Patienten

Bei 10 Patienten (35,7 \%) wurden folgende Störfaktoren erfasst: 7 Patienten hatten verletzte Nachbarfinger, zwei weitere klagten über Schulterschmerzen und in einem Fall wurde die verletzte Fingerkuppe zusätzlich mit Oberlin-Lappen rekonstruiert.

\begin{tabular}{|c|c|c|c|}
\hline \multirow{2}{*}{ DASH Score } & \multicolumn{3}{|c|}{ Patienten } \\
\cline { 2 - 4 } & $\mathbf{n}$ & MW \pm SD & R \\
\hline ADL & 18 & $6,2 \pm 7,6$ & $0-23,4$ \\
\hline Arbeit & 14 & $5,8 \pm 8,3$ & $0-25,0$ \\
\hline Sport & 14 & $10,3 \pm 18,4$ & $0-68,8$ \\
\hline
\end{tabular}

Tabelle 13: Der Mittelwert des DASH-Score für Patienten ohne Störfaktoren Ergebnisse $-67-$ 
Wie aus der Tabelle 13 hervorgeht, waren die DASH-Score Werte für Aktivitäten des täglichen Lebens und Arbeit bei Patienten ohne Störfaktoren im Vergleich zu allen Patienten um ca. $60 \%$ kleiner.

Die Ergebnisse des Vergleiches des DASH-Score mit Hilfe des Wilcoxon-Mann-WhitneyU-Tests zwischen Patienten mit und ohne Störfaktoren sind in der Tabelle 14 dargestellt. Es konnte kein statistisch signifikanter Unterschied nachgewiesen werden.

\begin{tabular}{|c|c|}
\hline DASH Score & p-Wert \\
\hline ADL & 0,07 \\
\hline Arbeit & 0,12 \\
\hline Sport & 0,48 \\
\hline
\end{tabular}

Tabelle 14: Mittelwerte DASH-Score zwischen allen Patienten und Patienten ohne Störfaktoren

\subsection{Nachuntersuchung von 24 Patienten mit 25 rekonstruierten Fingerkuppen durch} 27 neurovaskulären Insel-Lappen

\subsubsection{Lappengröße (Länge und Breite)}

Der mittlere Wert der Länge/Breite von 27 gemessenen Insel-Lappen lag bei 27,8 \pm 9,4 $\mathrm{mm} / 17,1 \pm 6,2 \mathrm{~mm}$. Die Spannweite der Messwerte von Länge/Breite betrug von 15 bis 60 $\mathrm{mm} /$ von 5 bis $35 \mathrm{~mm}$. Die Länge/Breite von größten Insel-Lappen war 60 x $35 \mathrm{~mm}$.

\subsubsection{Beweglichkeit des Handgelenkes}

Die Tabellen 15 und 16 zeigen die Beweglichkeit des gesunden und betroffenen Handgelenkes von 24 nachuntersuchten Patienten. Der Mittelwert der aktiven Beweglichkeit in Dorsalextension sowie Palmarflexion erreichte sowohl an der gesunden als auch an der betroffenen Seite ca. $68^{\circ}$. Das Gesamtausmaß der „Range of Motion“ der Extension/Flexion war im Mittel etwa 2,4 größer am gesunden als am betroffenen Handgelenk mit einer 
statistischen Signifikanz beim T-Test für paarweise Vergleiche $(\mathrm{p}=0,007)$. Ebenso unterscheidet sich die Flexion statistisch signifikant $(\mathrm{p}=0,03)$.

Der Mittelwert der Radialduktion lag bei ca. $40^{\circ}$ sowie die Ulnarduktion bei ca. $26^{\circ}$ beider Handgelenke und mit einem um 1,2 $2^{\circ}$ größeren Bewegungsausmaß der Radial-/Ulnarduktion am betroffenen Handgelenk. Es wurden kein statistisch signifikanter Unterschied dieser Parameter zwischen beiden Handgelenken nachgewiesen $(\mathrm{p}>0,05)$.

Der Mittelwert der Pronation sowie Supination des gesunden und betroffenen Unterarms betrug ca. $88,1 \pm 9,0^{\circ}$ mit einem ,Range of Motion“ der Pronation/Supination von ca. $176,3^{\circ}$.

\begin{tabular}{|c|c|c|c|c|}
\hline \multirow{2}{*}{$\begin{array}{l}\text { Aktive Beweglichkeit des } \\
\text { Handgelenkes/Unterarmes }\end{array}$} & \multicolumn{2}{|c|}{ gesunde Hand } & \multicolumn{2}{|c|}{ betroffene Hand } \\
\hline & $\mathbf{M W} \pm \mathbf{S D}$ & $\mathbf{R}$ & $\mathbf{M W} \pm \mathbf{S D}$ & $\mathbf{R}$ \\
\hline Extension $\left(^{\circ}\right)$ & $68,8 \pm 11,2$ & 50 bis 90 & $67,7 \pm 11,5$ & 55 bis 90 \\
\hline Flexion $\left(^{\circ}\right)$ & $67,7 \pm 12,6$ & 25 bis 90 & $66,3 \pm 12,5$ & 25 bis 90 \\
\hline ROM Extension/Flexion $\left({ }^{\circ}\right)$ & $136,4 \pm 18,1$ & 95 bis 160 & $134,0 \pm 18,5$ & 95 bis 160 \\
\hline Radialduktion $\left(^{\circ}\right)$ & $39,2 \pm 8,9$ & 25 bis 65 & $40,0 \pm 8,7$ & 35 bis 65 \\
\hline Ulnarduktion $\left(^{\circ}\right)$ & $25,6 \pm 7,4$ & 15 bis 40 & $26,0 \pm 7,5$ & 15 bis 40 \\
\hline ROM Radial/Ulnarduktion $\left(^{\circ}\right)$ & $64,8 \pm 12,8$ & 45 bis 95 & $66,0 \pm 12,9$ & 45 bis 95 \\
\hline Pronation $\left({ }^{\circ}\right)$ & $88,1 \pm 9,0$ & 45 bis 90 & $88,1 \pm 9,0$ & 45 bis 90 \\
\hline Supination $\left({ }^{\circ}\right)$ & $88,1 \pm 9,0$ & 45 bis 90 & $88,1 \pm 9,0$ & 45 bis 90 \\
\hline ROM Pronation/Supination $\left(^{\circ}\right)$ & $176,3 \pm 18,0$ & 90 bis 180 & $176,3 \pm 18,0$ & 90 bis 180 \\
\hline
\end{tabular}

Tabelle 15: Mittelwerte der aktiven Handbeweglichkeit für die jeweils gesunde und betroffene Hand 


\begin{tabular}{|c|c|}
\hline $\begin{array}{c}\text { Aktive Beweglichkeit des } \\
\text { Handgelenkes }\end{array}$ & p-Wert \\
\hline Extension $\left({ }^{\circ}\right)$ & 0,09 \\
\hline Flexion $\left(^{\circ}\right)$ & 0,03 \\
\hline ROM Extension/Flexion $\left({ }^{\circ}\right)$ & 0,007 \\
\hline Radialduktion $\left(^{\circ}\right)$ & 0,63 \\
\hline Ulnarduktion $\left(^{\circ}\right)$ & 0,66 \\
\hline ROM Radial/Ulnarduktion $\left({ }^{\circ}\right)$ & 0,63 \\
\hline
\end{tabular}

Tabelle 16: Unterschied der aktiven Beweglichkeit des gesunden und betroffenen Handgelenkes

\subsubsection{Beweglichkeit der Finger}

- Grundgelenk

Im Grundgelenk betrug der mittlere Wert der Beweglichkeit am verletzten Finger $86,7^{\circ} \pm$ 9,1 ${ }^{\circ}$. Es ergab sich keine signifikante Abweichung zur Gegenseite. Bei keinem Patienten bestand ein Streckdefizit im Grundgelenk.

- Proximales Interphalangealgelenk

Im proximalen Interphalangealgelenk betrug der mittlere Wert der Beweglichkeit am verletzten Finger 96,3 $\pm 6,2^{\circ}$. Es ergab sich keine signifikante Abweichung zur Gegenseite. Bei drei von 24 Patienten wurde ein Streckdefizit von $5^{\circ}, 15^{\circ}$ und $20^{\circ}$ im betroffenen Interphalangealgelenk dokumentiert.

- Distales Interphalangealgelenk

Im distalen Interphalangealgelenk betrug der mittlere Wert der Beweglichkeit am verletzten Finger $80,7^{\circ} \pm 19,0^{\circ}$. Es ergab sich keine signifikante Abweichung zur Gegenseite. Bei einem von 22 nachuntersuchten Patienten wurde zum Nachuntersuchungszeitpunkt ein Streckdefizit von $10^{\circ}$ gemessen.

- Total Active Motion

Der Mittelwert der „Total Active Motion“ am betroffenen Finger lag bei $260^{\circ} \pm 22,9^{\circ}$. Der Vergleich des TAM der beiden Fingerkuppen mittels t-Tests für paarweise Vergleiche ergab eine statistisch signifikante Differenz $(\mathrm{p}=0,02)$.

Ergebnisse 
Die Tabelle 17 veranschaulicht alle Werte der Flexion im Grund-, proximalen- und distalen Interphalangealgelenk sowie TAM der gesunden und betroffenen Finger.

Die statistische Auswertung mittels t-Tests für paarweise Vergleiche ergab einen signifikanten Unterschied (p-Werte zwischen 0,30 und 0,69).

\begin{tabular}{|c|c|c|c|c|}
\hline \multirow{2}{*}{ Gelenk } & \multicolumn{2}{|c|}{ betroffene Finger } & \multicolumn{2}{|c|}{ gesunde Finger } \\
\hline & MW \pm SD & $\mathbf{R}$ & $\mathbf{M W} \pm \mathbf{S D}$ & $\mathbf{R}$ \\
\hline GG-Flexion $\left(^{\circ}\right) \mathrm{n}=26$ & $86,7 \pm 9,1$ & 50,0 bis 90,0 & $87,7 \pm 7,0$ & 60,0 bis 90,0 \\
\hline PIP-Flexion $\left({ }^{\circ}\right)$ n=24 & $96,3 \pm 6,2$ & 80,0 bis 100,0 & $97,9 \pm 4,3$ & 85,0 bis 100,0 \\
\hline DIP-Flexion $\left({ }^{\circ}\right) \mathrm{n}=22$ & $80,7 \pm 19,0$ & 30,0 bis 90,0 & $83,9 \pm 14,9$ & 40,0 bis 90,0 \\
\hline TAM $\left(^{\circ}\right)$ & $260,0 \pm 22,9$ & 190,0 bis 280,0 & $268,4 \pm 15,1$ & 230,0 bis 280,0 \\
\hline
\end{tabular}

Tabelle 17: Die mittlere Werte der Flexion im Grund-, proximalen- und distalen Interphalangealgelenk sowie TAM der gesunden und betroffenen Finger

\begin{tabular}{|c|c|c|}
\hline Gelenk & $\begin{array}{c}\text { Vergleich zur } \\
\text { Gegenseite in \% }\end{array}$ & p-Wert \\
\hline GG-Flexion $\left(^{\circ}\right.$ ) $\mathbf{n = 2 6}$ & 99,0 & 0,7 \\
\hline PIP-Flexion $\left(^{\circ}\right.$ ) $\mathbf{n = 2 4}$ & 98,0 & 0,3 \\
\hline DIP-Flexion $\left(^{\circ}\right.$ ) $\mathbf{n = 2 2}$ & 96,0 & 0,6 \\
\hline TAM $^{\circ}\left(^{\circ}\right.$ & 97,0 & 0,02 \\
\hline
\end{tabular}

Tabelle 18: Unterschied der Flexion im Grund-, proximalen- und distalen Interphalangealgelenk sowie TAM der gesunden und betroffenen Finger

Der mittlere Wert der aktiven Beweglichkeit in DIP- und PIP-Gelenken von 22 betroffenen Fingern lag bei $174,1^{\circ} \pm 24,1^{\circ}$ mit einer Spannweite von $150^{\circ}$ bis $190^{\circ}$. Anhand der Strickland und Glogovac Kriterien entspricht der Wert einem exzellenten Ergebnis.

Bei einem Patienten wurde die Beugefähigkeit des betroffenen vs. gesunden Daumens im Grundgelenk und Interphalangealgelenk nachuntersucht. Die aktive Flexion im betroffenen Grundgelenk war um $35^{\circ}$ und im Interphalangealgelenk um $30^{\circ}$ kleiner. 
Der Kapandji-Index lag bei allen Patienten beidseits bei 10, was einer maximalen Beweglichkeit und Opposition des Daumenstrahlens entspricht.

\subsubsection{Kraftmessung der Finger}

Die Mittelwerte der Fingerkraft von 23 Patienten im Schlüsselgriff mit Hilfe des PinchGauges zwischen Daumen und den vier Fingerspitzen im Wechsel ist in der Tabelle 19 dargestellt. Dieser wurde bei allen Messungen an den gesunden Fingern gering größer gemessen. Am größten war der Unterschied am Zeige- und Ringfinger, um 1,1 kg bzw.1,2 $\mathrm{kg}$ und am kleinsten um 0,1 kg am Mittelfinger.

Mittels t-Tests für paarweise Vergleiche ergab sich keine statistisch signifikante Differenz (p-Werte zwischen 0,07 und 0,77).

\begin{tabular}{|c|c|c|c|c|}
\hline \multirow{2}{*}{ Pinch-Gauge (Kg) } & \multicolumn{2}{|c|}{ rekonstruierter Finger } & \multicolumn{2}{c|}{ Finger der Gegenseite } \\
\cline { 2 - 5 } & MW \pm SD & R & MW \pm SD & R \\
\hline D I - II (n=7) & $4,5 \pm 2,9$ & 2,5 bis 9,0 & $5,6 \pm 2,3$ & 3,0 bis 9,0 \\
\hline D I - III (n=10) & $4,1 \pm 2,3$ & 1,0 bis 9,5 & $4,0 \pm 1,5$ & 2,0 bis 7,0 \\
\hline D I - IV (n=4) & $4,6 \pm 2,2$ & 2,0 bis 7,5 & $5,8 \pm 4,3$ & 1,5 bis 11,0 \\
\hline D I - V (n=2) & $1,1 \pm 0,6$ & 0,5 bis 1,75 & $1,5 \pm 1,0$ & 0,5 bis 2,5 \\
\hline
\end{tabular}

Tabelle 19: Mittlere Werte bei der Messung der Fingerkraft am gesunden und betroffenen Finger mittels Pinch-Gauge

\subsubsection{Kraftmessung des Handgelenkes mit dem Jamar-Dynamometer Stufe 2}

Die Kraft des betroffenen Handgelenkes im Vergleich zur Gegenseite wurde mit dem Jamar-Dynamometer Stufe 2 bei 23 Patienten gemessen.

Die Tabelle 20 veranschaulicht die Werte der Kraftmessung der gesunden und betroffenen Hand bei drei Wiederholungen sowie den Mittelwert, das Maximum und Minimum. Die mittleren Werte lagen für die erste Messung bei 33,9 kg an der betroffenen Hand sowie bei $37,5 \mathrm{~kg}$ an der gesunden und wurden bis zur dritten Messung um rund 1,9 kg bzw. 2,9 kg 
geringer. Bei allen Messungen war die Kraft der gesunden Hand größer als die der betroffenen Hand.

Der Mittelwert von drei Messungen war 32,8 $\mathrm{kg}$ an der betroffenen und 36,0 $\mathrm{kg}$ an der gesunden Hand, was einen Kraftunterschien von ca. 8,8 \% ausmacht.

Der Vergleich der Kraftmessung zwischen der gesunden und betroffenen Hand ergab bei allen Messungen einen signifikanten Unterschied von $p<0,0001$ für die einzelnen Kraftmessungen sowie deren Mittelwert, Maximum und Minimalwert.

\begin{tabular}{|c|c|c|c|c|}
\hline \multirow{2}{*}{ Kraftmessung (kg) } & \multicolumn{2}{|c|}{ betroffene Hand } & \multicolumn{2}{c|}{ gesunde Hand } \\
\cline { 2 - 5 } & MW \pm SD & R & MW \pm SD & R \\
\hline $\mathbf{1}$ & $33,9 \pm 12,5$ & 14,0 bis 54,0 & $37,5 \pm 12,8$ & 18,0 bis 70,0 \\
\hline $\mathbf{2}$ & $32,6 \pm 13,0$ & 12,0 bis 56,0 & $35,9 \pm 11,8$ & 18,0 bis 62,0 \\
\hline $\mathbf{3}$ & $32,0 \pm 12,9$ & 10,0 bis 56,0 & $34,6 \pm 11,3$ & 18,0 bis 62,0 \\
\hline Mittelwert (1-3) & $32,8 \pm 12,6$ & 12,7 bis 50,7 & $36,0 \pm 11,8$ & 18,0 bis 64,7 \\
\hline Maximum & $35,0 \pm 13,2$ & 14,0 bis 56,0 & $38,3 \pm 12,6$ & 18,0 bis 70,0 \\
\hline Minimum & $30,8 \pm 11,9$ & 10,0 bis 50,0 & $34,1 \pm 11,5$ & 18,0 bis 62,0 \\
\hline
\end{tabular}

Tabelle 20: Mittlere Werte der Kraftmessung mittels Jamar-Dynamometer der gesunden und betroffenen Hand

\subsubsection{Zwei-Punkte-Diskrimination (2-PD)}

Bei allen gesunden Fingerkuppen lag die 2-PD zwischen 2 bis $6 \mathrm{~mm}$. Insgesamt zeigten 22 (81,5 \%) von 27 nachuntersuchten neurovaskulären Insel-Lappen ebenfalls Werte zwischen 2 bis $6 \mathrm{~mm}$. Bei 9 neurovaskulären Insel-Lappen (33,4\%) ergab sich kein Unterschied zwischen rekonstruierter und gesunder Seite. Wie in der Abbildung 30 dargestellt ist, lag die 2-PD bei 5 rekonstruierten Fingern (18,5\%) bei 7 bzw. $8 \mathrm{~mm}$. 


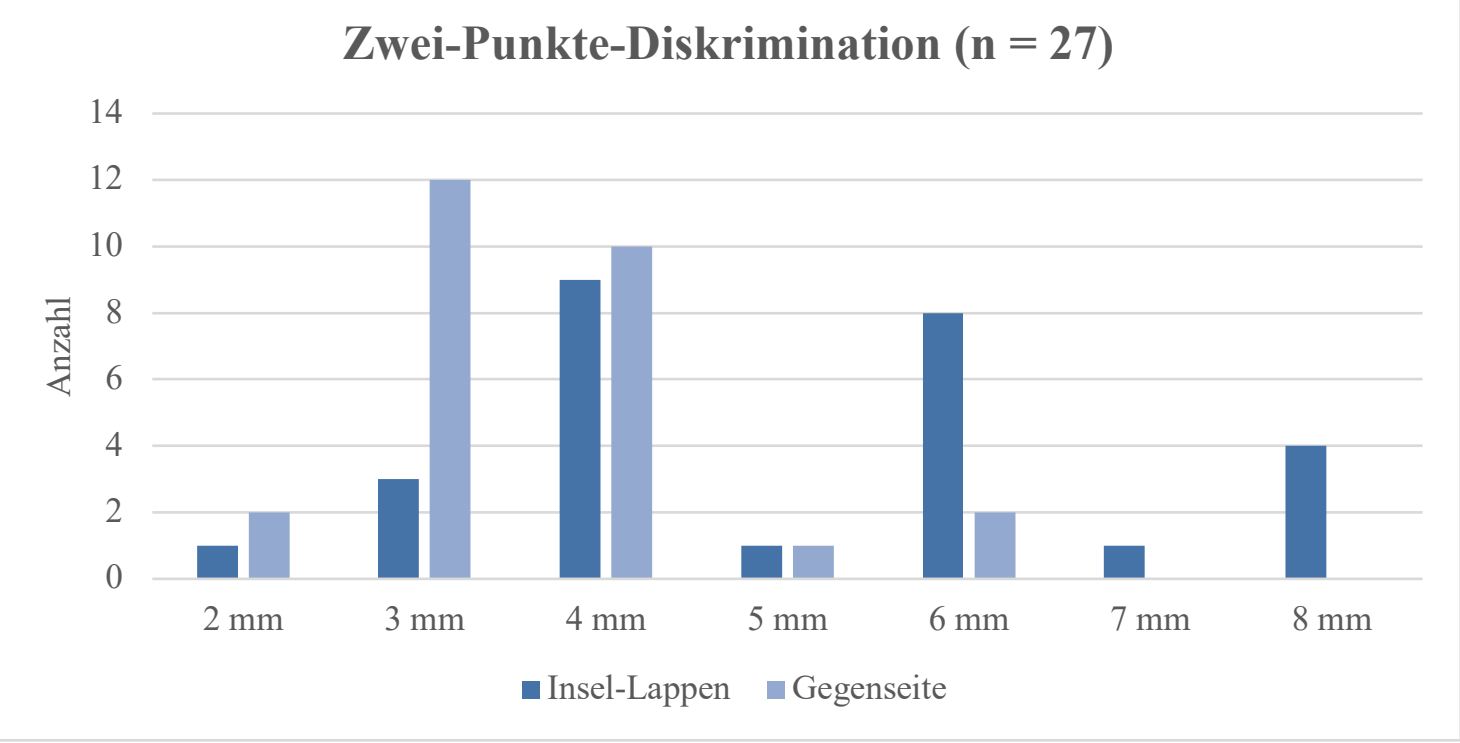

Abbildung 27: Auswertung der Zwei-Punkte-Diskrimination

Der mittlere Wert der 2-PD von 27 neurovaskulären Insel-Lappen lag bei 5,1 $\pm 1,7 \mathrm{~mm}$ mit einer Spannweite von 2 bis $8 \mathrm{~mm}$ und von der Fingerkuppe der Gegenseite bei 3,6 \pm 1,0 mm, mit einer Spannweite von 2 bis $6 \mathrm{~mm}$. Der Vergleich der beiden Fingerkuppen mittels tTestes für paarweise Vergleiche ergab eine statistisch signifikante Differenz $(p=0,005)$.

\subsubsection{Semmes-Weinstein Monofilament-Test (SWMT)}

Die Tabelle 21 und Abbildung 28 zeigen den Vergleich der Werte des SWMT von 27 neurovaskulären Insel-Lappen zur gegenüberliegenden gesunden Fingerkuppe.

Bei 16 neurovaskulären Insel-Lappen (59,3 \%) betrug der Wert 2,83N, was einer normalen Sensibilität entspricht und bei $9(33,4 \%)$ eine eingeschränkte Berührungsempfindung mit einem Wert von 3,61N.

Die Ergebnisse waren bei insgesamt fünf Insel-Lappen (18,5\%) schlechter als an der Fingerkuppe der Gegenseite. 


\begin{tabular}{|c|c|c|c|}
\hline \multicolumn{2}{|c|}{ Insel-Lappen } & \multicolumn{2}{c|}{ Gegenseite } \\
\hline SWM & $\mathbf{n}$ & SWM & n \\
\hline $2,83 \mathrm{~N}$ & 16 & $2,83 \mathrm{~N}$ & 15 \\
\hline $3,61 \mathrm{~N}$ & 9 & $3,61 \mathrm{~N}$ & 12 \\
\hline $4,31 \mathrm{~N}$ & 2 & $4,31 \mathrm{~N}$ & 0 \\
\hline
\end{tabular}

Tabelle 21: Werte des Semmes-Weinstein Monofilament-Testes der neurovaskulären InselLappen und Fingerkuppe der Gegenseite

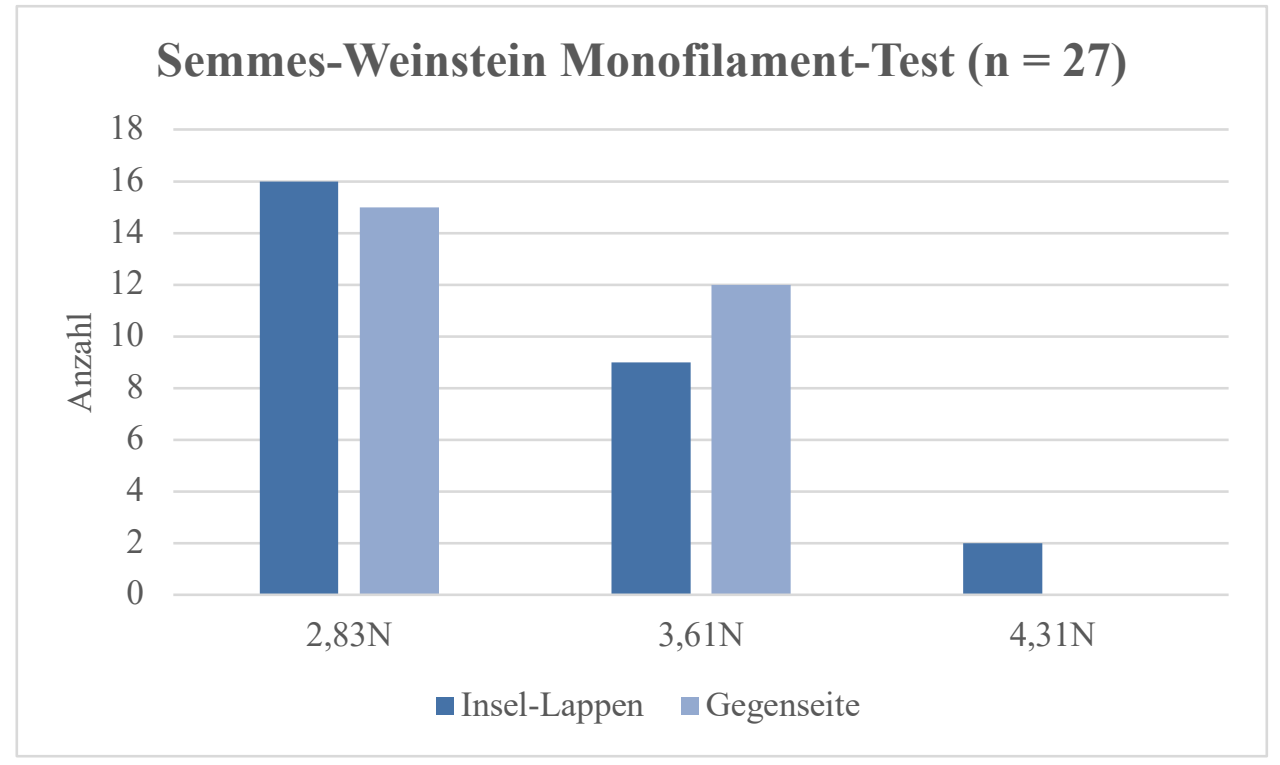

Abbildung 28: Auswertung des Semmes-Weinstein Monofilament-Testes

Zur statistischen Auswertung wurde der SWMT in einen Score umgewandelt und mit Hilfe des Wilcoxon-Mann-Whitney-U-Tests für unabhängige Stichproben ausgewertet. Es konnte kein statistisch signifikanter Unterschied zwischen neurovaskulären Insel-Lappen und der Gegenseite nachgewiesen werden $(\mathrm{p}=0,976)$.

\subsubsection{Narbenbeschreibung und Vancouver Scar Scale (VSS)}

Die Abbildung 29 veranschaulicht die Ergebnisse der Narbenbeschaffenheit bewertet durch die VSS. Der Mittelwert der VSS bei allen Patienten lag bei 0,6 \pm 0,9. Bei 17 Patienten (60,7 
\%) wurde die Narbe mit einer Punktezahl von 0 bewertet, was annähernd der normalen Haut entspricht. Bei 26 (92,3 \%) von insgesamt 28 nachuntersuchten Fingerkuppen wurde die Narbe vom Untersucher als „weich“ beschrieben. Die beiden am schlechtesten bewerteten Narben (VSS von 3) wurden einmal als „,verhärtet“ und einmal als „,berührungsempfindlich sowie verhärtet" dokumentiert.

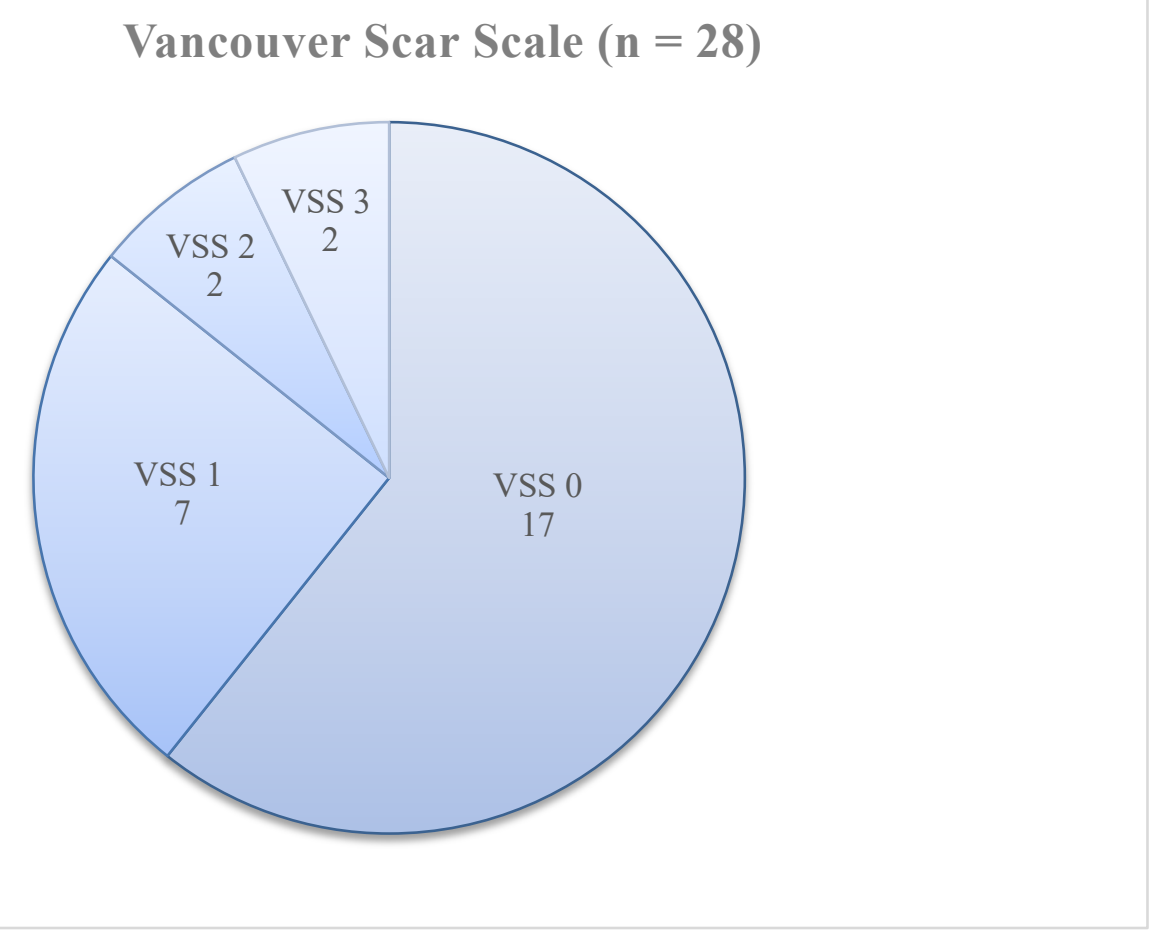

Abbildung 29: Auswertung der Vancouver Scar Scale

\subsubsection{Nagelwachstumsstörungen}

Von 29 rekonstruierten Fingerkuppen konnte bei 22 (75,9 \%) der Fingernagel erhalten werden.

Die häufigste Nagelwachstumsstörung in Form eines Krallennagels wurde in 14 Fällen (63,6 \%) dokumentiert. Nach der Lim-Klassifikation für Krallennägel weisen 6 Patienten (42,9 \%) Grad 1, 7 Patienten (50,0 \%) Grad 2 und ein Patient (7,1 \%) Grad 3 auf.

Bei einem Patienten $(4,5 \%)$ wurde der Nagel an dem rekonstruierten Finger als dünn beschrieben.

Ergebnisse

$-76-$ 
Nagelverkürzungen von mehr als $2 \mathrm{~mm}$ im Vergleich mit der gesunden Gegenseite traten bei 3 Patienten $(13,6 \%)$ auf. Der mittlere Wert der Fingerlänge an der rekonstruierten Fingerkuppe lag bei 11,9 2,9 mm mit einer Spannweite von 6 bis $16 \mathrm{~mm}$. Die Länge der Nägel am gesunden Finger betrug 12,2 $\pm 2,3 \mathrm{~mm}$ mit einer Spannweite von 8 bis $16 \mathrm{~mm}$. Der Unterschied der Fingerlänge beider Gruppen wurde mittels t-Test bei unabhängigen Stichproben als statistisch signifikant $(p<0,05)$ nachgewiesen.

Bei keinem der Patienten trat bis zum Untersuchungszeitpunkt eine Entzündung des Nagels der betroffenen Seite auf.

\subsection{Fotodokumentation der Finger im Vergleich zur gesunden Seite}

Die Bilder auf Abbildung 30 und 31 zeigen eine hervorragende Weichteildeckung der Fingerkuppenverletzungen mit freiliegenden Knochen bei unseren Patienten durch neurovaskuläre Insel-Lappen. 


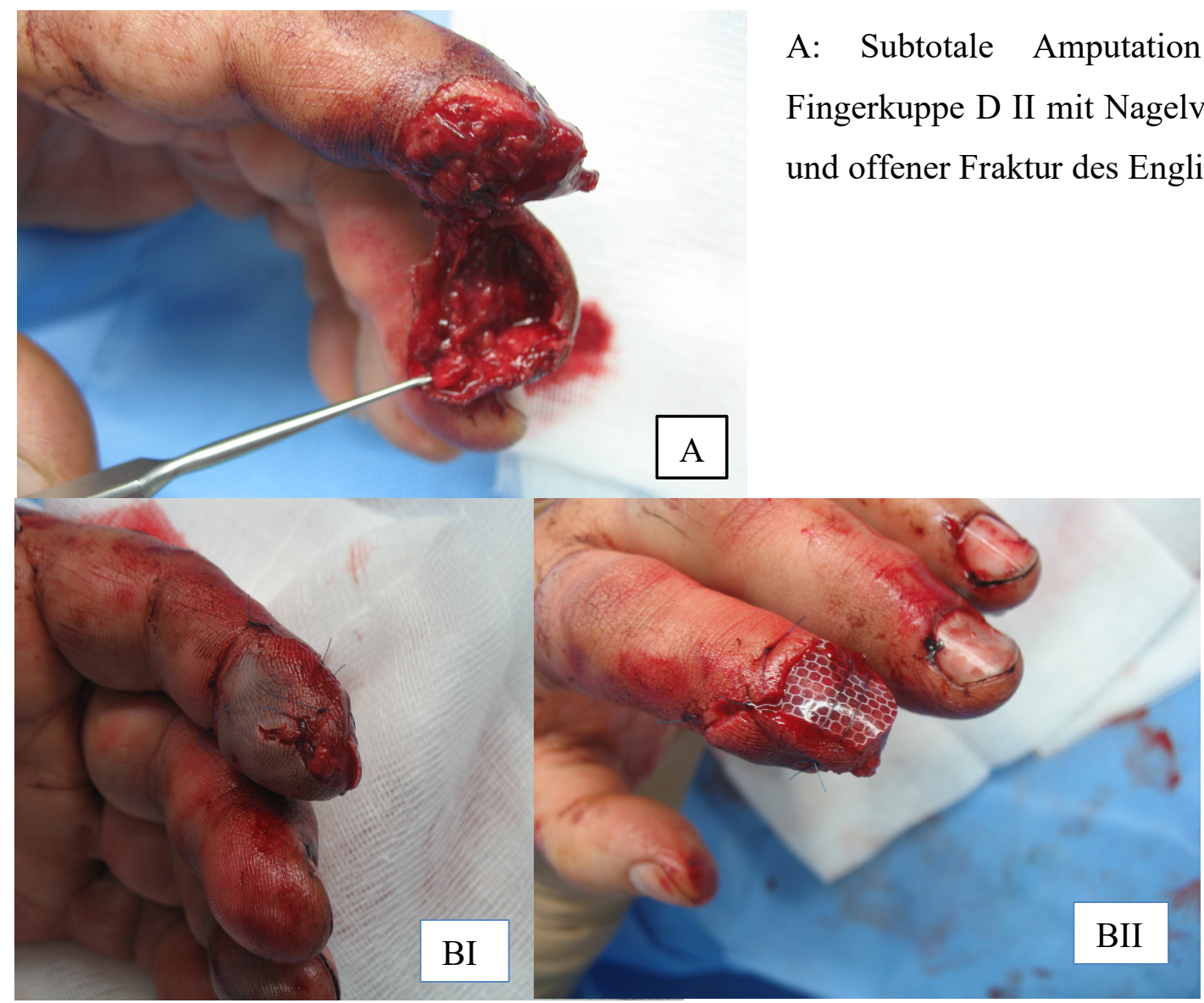

B I-II: Ulnar gestielter neurovaskulärer Insel-Lappen
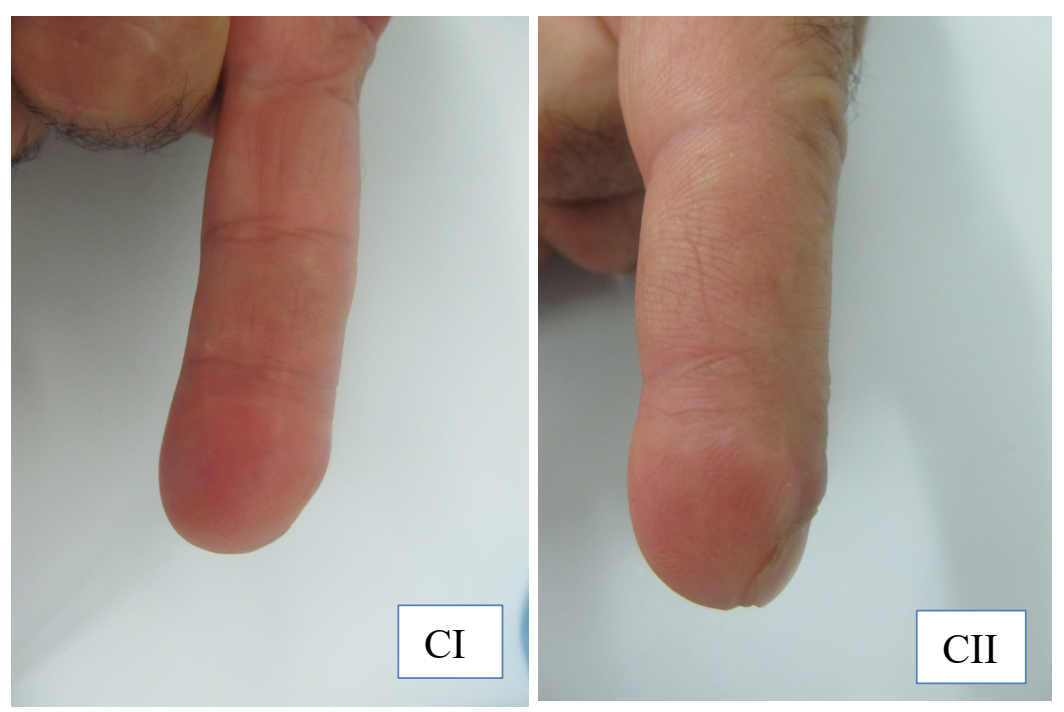

C I-II: Ergebnis

nach

54

Monaten

Abbildung 30: Pre-, intra- und postoperative Bilder einer Fingerkuppenverletzung am Zeigerfinger

Ergebnisse

$-78-$ 

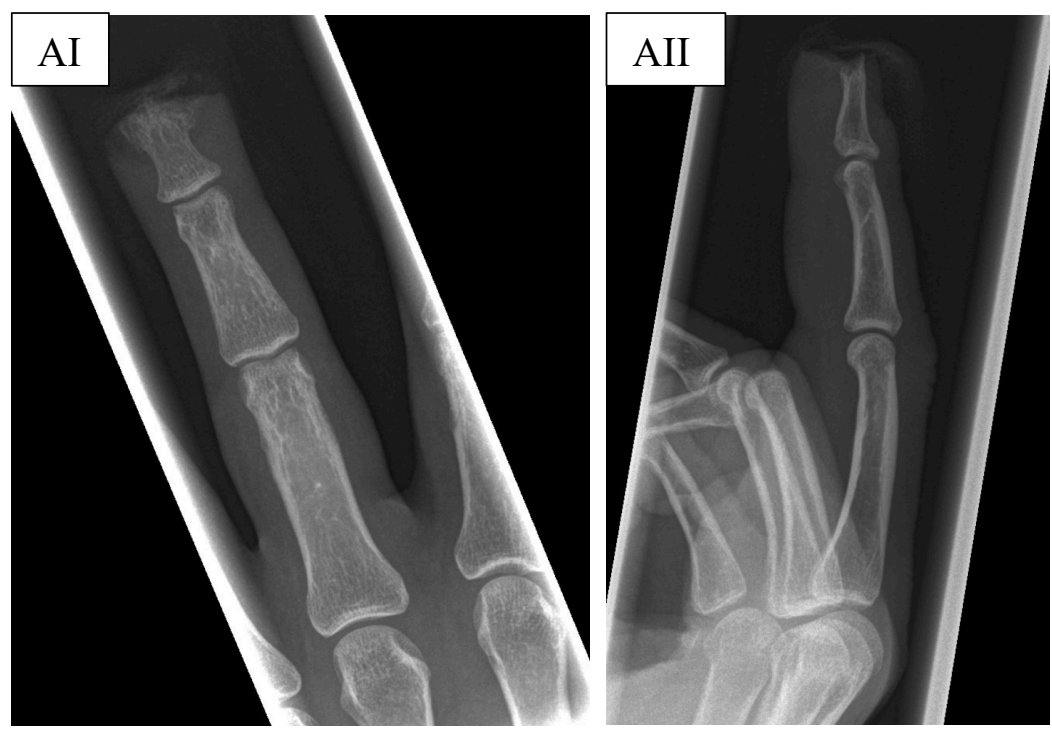

A I-II: Fraktur der distalen Phalanx des Mittelfingers

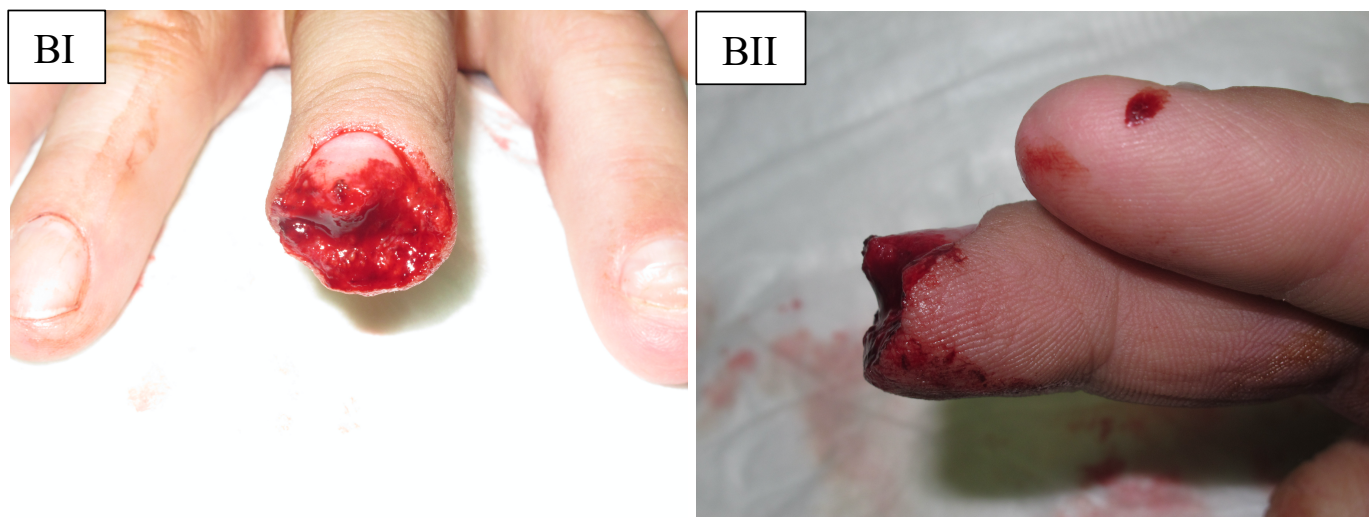

B I-II: Subtotale Amputation der Fingerkuppe D III mit offener Fraktur des Engliedes

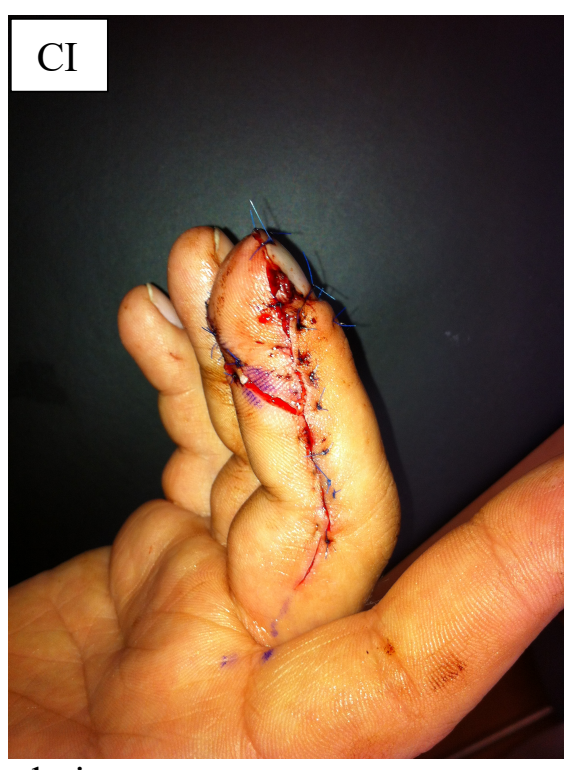

Ergebnisse

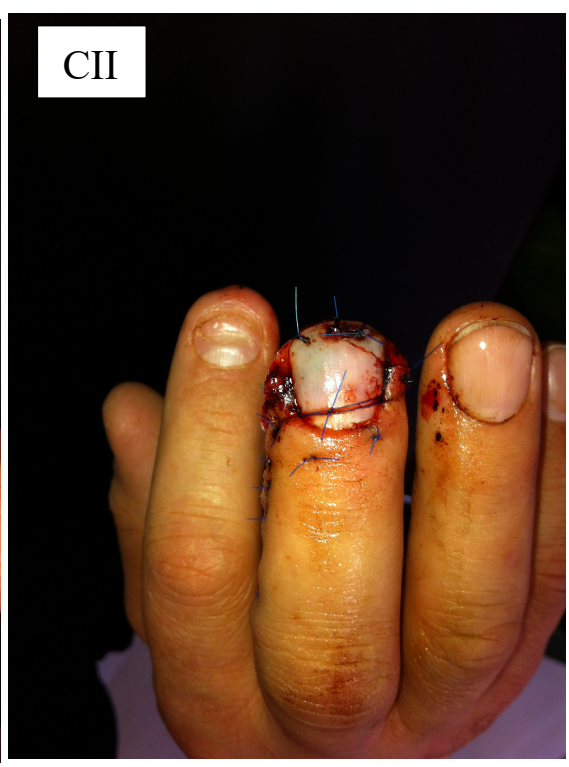

$-79-$
C I-II: Radial gestielter neurovaskulärer Insel-Lappen 

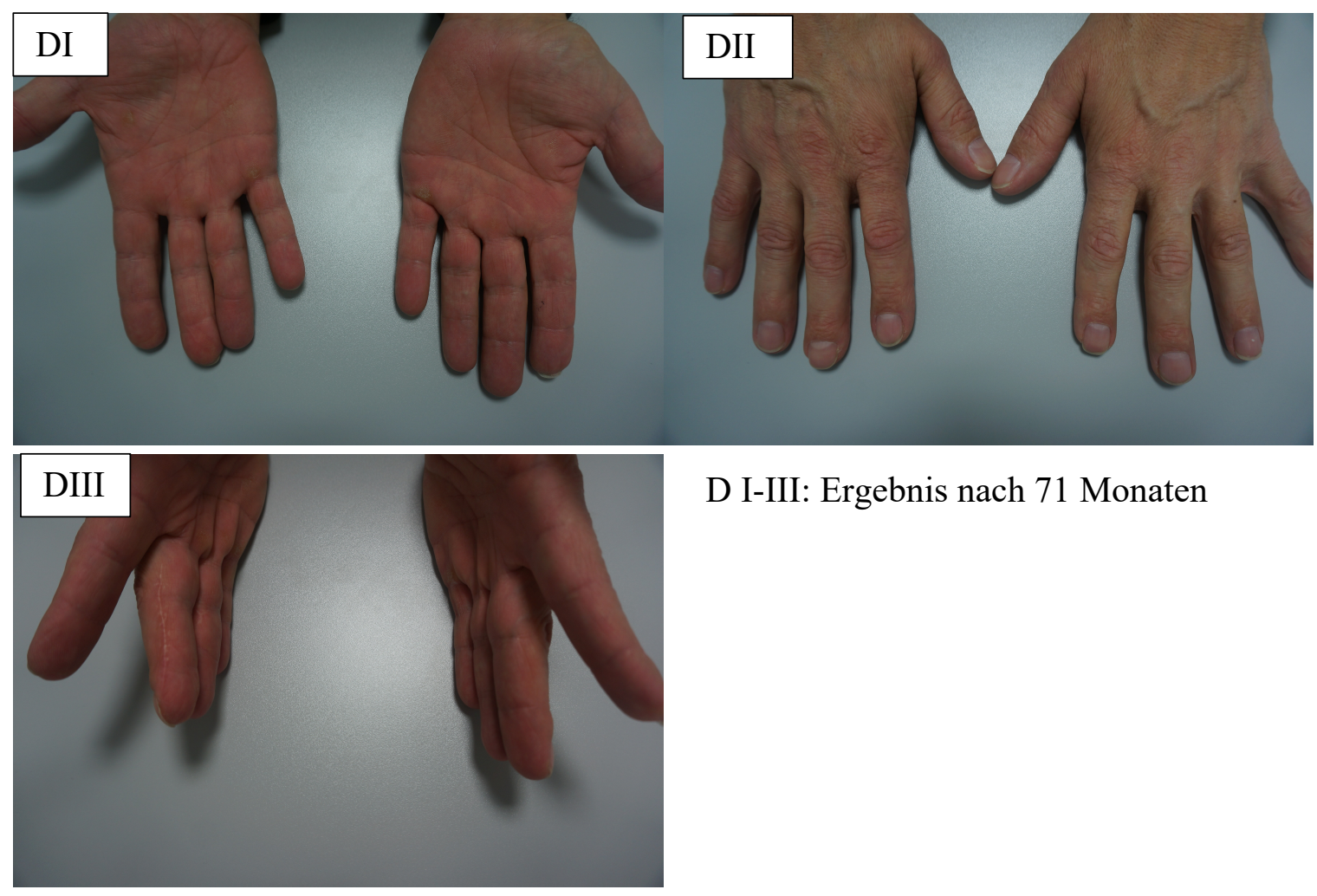

D I-III: Ergebnis nach 71 Monaten

Abbildung 31: Röntgen-, pre-, intra- und postoperative Bilder einer Fingerkuppenverletzung am Mittelfinger 


\section{Diskussion}

Fingerkuppenverletzungen sind ein häufiger Grund für die Vorstellung in der Notaufnahme 1-3,64 und stellen oft eine Herausforderung für den Chirurgen dar. In der Literatur wurden unterschiedliche Klassifikationen ${ }^{31-37,104}$ und Therapieschemata ${ }^{4,38}$ diskutiert. Die Rekonstruktion der Fingerkuppenverletzungen mittels neurovaskulären Insel-Lappen zeigte in vorherigen Publikationen oft gute Ergebnisse. ${ }^{72,150,151}$ Die Mehrheit dieser Studien beschreiben jedoch nur kurz- oder mittelfristige Ergebnisse. ${ }^{115,152-155}$ Die Erfahrung von Langzeit-Ergebnissen könnte dem Handchirurgen helfen, eine richtige Wahl der Therapie unter Berücksichtigung der individuellen Situation des Patienten zu treffen.

In der vorliegenden Studie sind die Langzeit-Ergebnisse nach der Rekonstruktion mittels homodigitalen neurovaskulären Insel-Lappenplastiken dargestellt. Zuerst erfolgte eine

Auswertung der Patientenakten von insgesamt 53 Patienten mit 57 Fingerkuppenverletzungen, davon konnten 28 Patienten mit 29 Fingerkuppenverletzungen nachuntersucht werden.

Die mittlere Nachuntersuchungsdauer betrug mehr als 8 Jahre (105 Monate) mit einer Spannweite von 42 bis 169 Monaten.

\subsection{Demographie}

Die untersuchten Patienten hatten zum Zeitpunkt der Operation ein Durchschnittsalter von 38,4 Jahren. Es handelte sich überwiegend um männliche $(88,7 \%)$ und berufstätige $(96,4$ \%) Patienten. So zeigte sich in einer retrospektiven Untersuchung von Hamdi ${ }^{154}$ über 31 Patienten und 32 Defektdeckungen mit homodigitalen neurovaskulären Insel-Lappen, dass es sich bei seinen Patienten vorwiegend um junge männliche Patienten handelte, welche handwerklich tätig waren. Auch in anderen Publikationen erlitten in $72 \%$ bis 92,7 \% männliche Patienten im durchschnittlichen Alter von 31,4 bis 38 Jahren $^{72,152,156,157}$ eine Fingerkuppenverletzung.

Die Mehrheit der Fälle in unserer Studie, nämlich 73,6\%, geschah in Folge eines Arbeitsunfalls. Eine ähnliche Häufigkeit von Arbeitsunfällen wurde mit 76,5\% in der Arbeit von Borman ${ }^{158}$ beschrieben. Bei den anderen Autoren wurde eine arbeitsbedingte Fingerkuppenverletzung zwischen $61 \%$ und $100 \%$ dokumentiert. ${ }^{153,155-157}$

Diskussion 
Daraus ergibt sich, dass eine markante Mehrheit der Fingerkuppenverletzungen in Folge eines Arbeitsunfalles bei männlichen Patienten des mittleren Alters zu verzeichnen war. Gerade bei diesen Patienten musste mit der plastisch-chirurgischen Defektdeckung ein gutes funktionelles Resultat, besonders für die Fortsetzung der beruflichen Tätigkeit, erzielt werden. ${ }^{159}$

\subsection{Lokalisation und Klassifikation der Fingerkuppenverletzungen}

In unserer Studie traten am häufigsten die Verletzungen am Mittelfinger mit 38,3\%, gefolgt vom Zeigefinger mit 29,6 \% und Ringfinger mit 22,2 \% auf. Eine ähnliche Prozentzahl der Fingerkuppenverletzungen am Mittelfinger mit 39,3 \% wurde in der Studie von Kleinert ${ }^{94}$ dokumentiert. Auch in den anderen Studien waren Fingerkuppen am Zeige- oder Mittelfinger am häufigsten betroffen. ${ }^{115,155,160}$

Schon Zook ${ }^{161}$ konnte in seiner Arbeit an 299 Fingern eine Korrelation zwischen der Prominenz von Langfingern und dem Schweregrad der Fingerkuppenverletzung feststellen. Hier wurden insgesamt 96 Mittelfinger (32,1\%) wegen seiner herausragenden Position am häufigsten verletzt.

Bei $56 \%$ unserer Patienten war die dominierende Hand zugleich die verletzte Hand. Auch in den Studien von Tsai ${ }^{150}$ war die dominante Hand in $68,7 \%$ und von Cook $^{157}$ in 78,9 \% verletzt.

Es lässt sich erkennen, dass am häufigsten die dominierende Hand und die Längsfinger eine Fingerkuppenverletzung erleiden. ${ }^{159}$

Alle Fingerkuppenverletzungen in unserer Studie wurden nach der Allen-Klassifikation in Zone III mit $86 \%$ und IV mit $14 \%$ eingeteilt.

Der Durchmesser der neurovaskulären Insel-Lappen bei unseren Patienten zum Zeitpunkt der Nachuntersuchung betrug von $0,5 \times 1,5 \mathrm{~cm}$ bis zu 3,5 x 6,0 cm. Die ursprüngliche Defektgröße der Insel-Lappen war noch größer und wurde durch die Kontrakturbildung verkleinert. In anderen Arbeiten lag der Durchmesser der neurovaskulären Insel-Lappen von 1,5 bis zu 5 cm. ${ }^{72,155,157}$

Diskussion $-82-$ 
In der Arbeit von Magerle ${ }^{162}$ wird die Rekonstruktion der Fingerkuppenverletzungen mittels Cross-Finger-Lappen mit einem Durchmesser nicht größer als 2,5 x $2 \mathrm{~cm}$ empfohlen.

Demnach erlaubt die neurovaskuläre Insel-Lappenplastik eine Versorgung von großflächigen Fingerkuppenverletzungen mit freiliegenden Sehnen bzw. Knochen, ${ }^{56,57,114,155}$ welche nach Allen-Klassifikation in Zone III und IV ${ }^{72}$ unterteilt sind.

\subsection{Dauer des stationären Aufenthaltes und der Arbeitsunfähigkeitsdauer}

Die mittlere Dauer des stationären Aufenthaltes lag bei 5,6 Tagen mit einer Spannweite von 0 bis 14 Tagen. Keine anderen Studien gingen auf die stationäre Aufenthaltsdauer ein ${ }^{72,115,155-}$ 157.

Die Tabelle 22 veranschaulicht eine Behandlungsdauer mittels Semiokklusionsverband bei Fingerkuppenverletzungen nach Allen-Klassifikation Zone I bis III gegenüber unserer Studie. Die durchschnittliche Behandlungsdauer bei Okklusionstherapie lag bei ca. 2 bis 6,5 Wochen $^{50-53,163}$ und erforderte einen häufigen Verbandswechsel sowie eine hohe Patientencompliance. Die Arbeit von Quadlbauer ${ }^{55}$ und Kollegen zeigt überwiegend eine längere Behandlungsdauer bei Patienten mit schweren Verletzungen, welche mittels Semiokklusionsverband versorgt wurden. Hier lag die Behandlungsdauer bei Fingerkuppenverletzungen nach der Allen-Klassifikation Zone III bei 4,1 Wochen und Zone IV bei 6 Wochen.

Diskussion $-83-$ 


\begin{tabular}{|c|c|c|c|c|c|}
\hline Studie & 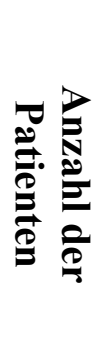 & 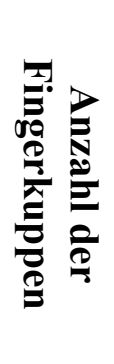 & 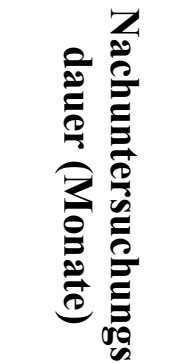 & 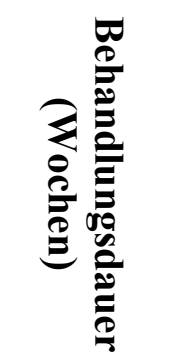 & 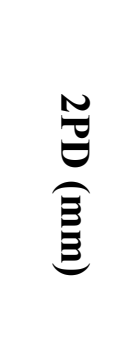 \\
\hline $\begin{array}{l}\text { Mennen und } \\
\text { Wiese } 51\end{array}$ & 200 & 200 & 3 & 3 bis 4,2 & 2,5 \\
\hline $\begin{array}{l}\text { Quell }{ }^{53} \text { und } \\
\text { Kollegen }\end{array}$ & 42 & 42 & 2 bis 6 & 2 bis 6 & 2 bis 8 \\
\hline $\begin{array}{l}\text { Hoigné }{ }^{52} \text { und } \\
\text { Kollegen }\end{array}$ & 17 & 19 & 6 bis 12 & 6,5 & 4,3 \\
\hline $\begin{array}{l}\text { Dereskewitz }{ }^{163} \\
\text { und Kollegen }\end{array}$ & 100 & 100 & - & 3 & 5,8 \\
\hline Eigene Studie & 28 & 29 & 42 bis 169 & 0 bis 2 & 2 bis 8 \\
\hline
\end{tabular}

Tabelle 22: Ergebnisse der Behandlungsdauer von Fingerkuppenverletzungen mit einem Semiokklusionsverband

Die arbeitsfähigen Patienten konnten nach ca. 6,1 Wochen mit einer Spannweite von 1 bis 12 Wochen zu ihrer Arbeit zurückkehren. Keiner der nachuntersuchten Patienten musste infolge der Verletzung seinen bisher ausgeübten Beruf wechseln.

In der Literatur ist mit einer ähnlichen Dauer der Arbeitsunfähigkeit nach einer Rekonstruktion von Fingerkuppenverletzungen mit lokalen Lappenplastiken im Durchschnitt von 6 bis 12 Wochen $\mathrm{zu}$ rechnen. ${ }^{43,57,68,70,76,164-167}$ Eine etwas kürzere Arbeitsunfähigkeitsdauer mit 5 Wochen wurde bei einer Behandlung mittels Folientherapie dokumentiert. ${ }^{163}$ Das kann daran liegen, dass mittels Folienverband bisher vorwiegend die leichteren Verletzungen der Fingerkuppen, Allen-Klassifikation Zone I und II, versorgt werden.

In der Arbeit von Quadlbauer ${ }^{55}$ und Kollegen wurde in 9 Fällen eine Rekonstruktion der Fingerkuppenverletzungen mittels Okklusionsverband nach Allen-Klassifikation Zone III mit einer mittleren Arbeitsunfähigkeitsdauer von 6,4 Wochen und in 4 Fällen nach AllenKlassifikation Zone IV mit 8,3 Wochen beschrieben. Somit scheint die Dauer der Arbeitsunfähigkeit primär vom Schweregrad sowie der psychosozialen Auswirkung der Diskussion 
Fingerkuppenverletzung beeinflusst zu werden, wohingegen die Behandlungsmethode sekundär zu seien scheint. ${ }^{168-170}$

\subsection{Patientenzufriedenheit, ästhetisches Outcome und DASH-Score.}

Alle befragten Patienten würden sich wieder operieren lassen und waren mit dem Operationsergebnis hoch zufrieden. Als häufigster Grund dafür wurde die Erhaltung der Fingerlänge, die weiterhin bestehende Funktionalität der Hand und nicht zuletzt ein gutes ästhetisches Ergebnis genannt. Die mittlere Patientenzufriedenheit fiel mit 8,7 der NRS sehr positiv aus.

Die Tabelle 23 veranschaulicht eine hohe Patientenzufriedenheit mit der Funktionalität und ästhetischem Outcome nach der Rekonstruktion mittels neurovaskulärer Insel-Lappenplastik in den anderen Studien.

\begin{tabular}{|c|c|c|c|}
\hline Studie & 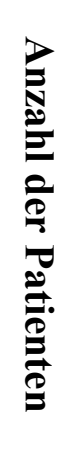 & 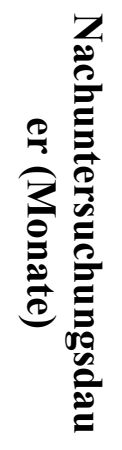 & 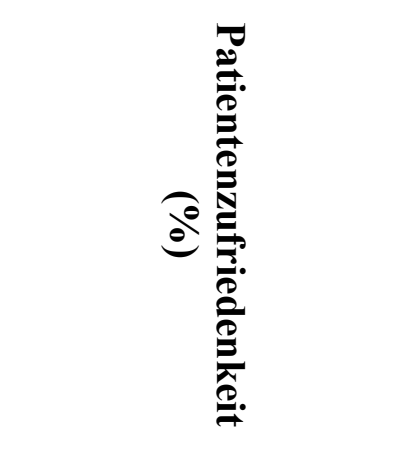 \\
\hline Varitimidis und Dailiana $^{72}$ & 50 & 46 & 100 \\
\hline Adani $^{171}$ und Kollegen & 32 & 27 & $\begin{array}{c}100 \text { (72 hochzufrieden, } \\
28 \text { zufrieden) }\end{array}$ \\
\hline Lanzetta $^{152}$ und Kollegen & 25 & 30 & 96 \\
\hline Kayalar $^{156}$ und Kollegen & 96 & 41 & 91,7 \\
\hline Tsai ${ }^{150}$ und Kollegen & 16 & 26 & 88 \\
\hline
\end{tabular}

Tabelle 23: Ergebnisse der Patientenzufriedenheit nach der Rekonstruktion von Fingerkuppenverletzungen mittels neurovaskulärer Insel-Lappenplastik

Eine hohe Patientenzufriedenheit wurde ebenfalls nach der Therapie mittels Okklusionsverband beobachtet. ${ }^{52,65,163}$ 
Der DASH Score ergab in unserer Patientengruppe gute Ergebnisse. Der mittlere DASH Score für die Aktivitäten des täglichen Lebens lag bei 6,2, für die Arbeit bei 5,8 und für den Sport bei 10,3.

In einer Vergleichsstudie von Nakanishi ${ }^{172}$ und Kollegen wurden die Ergebnisse der Rekonstruktion von 23 Fingerkuppenverletzungen mittels neurovaskulärer homodigitaler Insel-Lappenplastik und 14 Fingerkuppenverletzungen der mikrochirurgischen Replantation beschrieben. Die mittlere Nachbeobachtungszeit bei der ersten Gruppe betrug 44 Monate und bei der zweiten Gruppe 25 Monate. Der DASH Score ergab ein gutes Ergebnis in beiden Gruppen mit einem Mittelwert von 7 für die neurovaskuläre Insel-Lappenplastik und 8 für die mikrochirurgische Replantation. Die Auswertung des DASH-Fragenbogens in der Studie von Dereskewitz ${ }^{163}$ und Kollegen bei 100 Fingerkuppenverletzungen nach der Therapie mittels Folienverband ergab einen mittleren DASH Score von 13,9 Punkten. Eine genaue Nachbeobachtungszeit wurde nicht angegeben.

Es lässt sich erkennen, dass die Rekonstruktion von Fingerkuppenverletzungen mittels operativer neurovaskulärer Insel-Lappenplastik als auch konservativer Okklusionstherapie eine hohe Patientenzufriedenheit und einen guten DASH-Score zeigen.

\subsection{Narbenbeschaffenheit, Weichteildeckung und Schmezempfindlichkeit}

Bei unseren Patienten wurde die Narbe in $92,3 \%$ als weich beschrieben und in $60,7 \%$ entsprach die Narbengegebenheit anhand der Vancouver Scar Scale annähend der normalen Haut.

In einer Studie von Lanzetta ${ }^{152}$ und Kollegen mit einer mittleren Nachbeobachtungsdauer von 30 Monaten wurde insgesamt bei $12 \%$ der Fingerkuppen nach einer Rekonstruktion mittels neurovaskulären Insel-Lappen eine überempfindliche Narbe gebildet. Kayalar ${ }^{156}$ und Kollegen beschreiben in ihrer Arbeit bei 6,1\% rekonstruierten Fingerkuppen die Bildung einer hypertrophen Narbe nach einer mittleren Nachbeobachtungzeit von 41 Monaten.

Dies kann in anderen Studien bestätigt werden. ${ }^{152}$

In den letzten Jahren verzeichnet die konservative Behandlung von Fingerkuppenverletzungen mittels Semiokklusionsverband eine steigende Tendenz. $\mathrm{Zu}$ den Vorteilen dieser Technik zählt die Wiederherstellung von Form und eine suffiziente Diskussion -86 - 
Weichteilpolsterung der Fingerkuppe, fast narbenfreie Regeneration mit Ausbildung der Papillarleisten und gute Sensibilität. ${ }^{49,52,53,163}$

Insgesamt gaben $75 \%$ der nachuntersuchten Patienten in Ruhe und 53,6 \% unter Belastung keine Schmerzen an. Der mittlere Wert der Schmerzen anhand der NRS lag in Ruhe bei 1,2 und unter Belastung unter 2,4.

In der Studie von Varitimidis und Dailiana ${ }^{72}$ konnte bei keiner der 63 durch neurovaskuläre Insel-Lappen versorgten Fingerkuppenverletzungen nach einer mittleren Nachbeobachtungzeit von 46 Monaten eine Schmerzempfindlichkeit oder empfindliche Narbe beobachtet werden. In einer Langzeitstudie von Tsai ${ }^{150}$ und Kollegen mit einer mittleren Nachbeobachtungsdauer von 62 Monaten und einer Rekonstruktion von 16 Fingerkuppendefekten haben 43,7 \% der Patienten leichte Schmerz angegeben. In der Studie von Lok ${ }^{155}$ und Kollegen mit einer mittleren Nachbeobachtungszeit von 3 Monaten wurde eine Schmerzempfindlichkeit bei 16,7 \% Patienten beschrieben.

Hiernach erzielt die neurovaskuläre Insel-Lappenplastik eine narbenkontrakturfreie Weichteilpolsterung der Fingerkuppen mit einer tolerablen Schmerzempfindlichkeit. Die obengenannten Parameter unterliegen oft der Therapie mittels Folienverband, wenngleich durch neurovaskuläre Insel-Lappenplastik meistens schwerere Fingerkuppenverletzungen versorgt werden als durch Okklusionstherapie.

\subsection{Beweglichkeit}

Der Erhalt der normalen aktiven Beweglichkeit ohne Entstehung eines Streckdefizites ist ein wichtiges Ziel einer suffizienten Fingerkuppenrekonstruktion.

In unserer Arbeit zeigten sich im Grundgelenk keine Bewegungseinschränkungen und im distalen Interphalangealgelenk zeigte lediglich ein (4,5\%) von 22 rekonstruierten Fingern ein Streckdefizit von $10^{\circ}$.

Im proximalen Interphalangealgelenk entwickelte sich bei drei (12,5\%) von 24 Patienten ein Streckdefizit von $5^{\circ}, 15^{\circ}$ und $20^{\circ}$. Eine standardisierte Verwendung einer Streckschiene bei unseren Patienten fand nicht statt.

Die „Total Active Motion“ ergab bei unseren Patienten einen Wert über 97 \% zur gesunden Gegenseite, was nach Kleinert ${ }^{136}$ als ,gut“ zu bewerten ist.

Diskussion

-87 - 
In Tabelle 24 ist ein Überblick des Streckdefizits im PIP und DIP Gelenk nach der Rekonstruktion von Fingerkuppenverletzungen mittels neurovaskulärer Insel-Lappenplastik.

\begin{tabular}{|c|c|c|c|c|c|c|}
\hline \multirow[b]{2}{*}{ Studie } & \multirow[b]{2}{*}{ 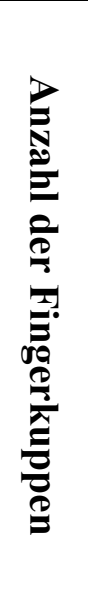 } & \multirow[b]{2}{*}{ 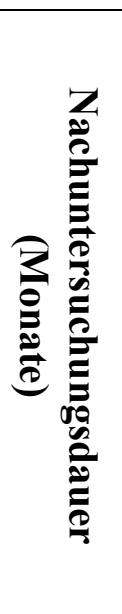 } & \multicolumn{2}{|r|}{ PIP } & \multicolumn{2}{|r|}{ DIP } \\
\hline & & & 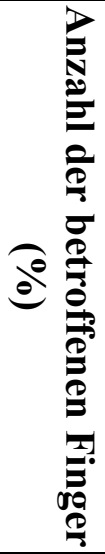 & 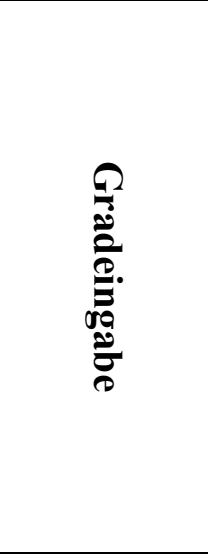 & 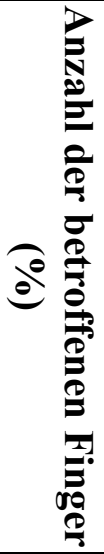 & 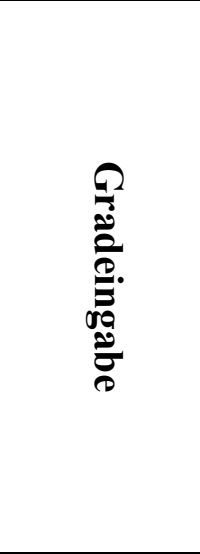 \\
\hline $\begin{array}{l}\text { Eigene } \\
\text { Studie }\end{array}$ & 29 & 105 & 12,5 & $5^{\circ}, 15^{\circ}, 20^{\circ}$ & 4,5 & $10^{\circ}$ \\
\hline $\begin{array}{l}\text { Varitimidis } \\
\text { und } \\
\text { Dailiana }^{72}\end{array}$ & 63 & 46 & - & 0 & 23 & $10^{\circ}$ bis $20^{\circ}$ \\
\hline $\begin{array}{l}\text { Kayalar }^{156} \\
\text { und } \\
\text { Kollegen }\end{array}$ & 115 & 41 & 6,1 & $25^{\circ}$ & 0 & 10 015 20 \\
\hline $\begin{array}{l}\text { Usami }^{160} \\
\text { und } \\
\text { Kollegen }\end{array}$ & 17 & 12,5 & 4 & - & - & - \\
\hline $\begin{array}{l}\text { Adani }{ }^{171} \\
\text { und } \\
\text { Kollegen }\end{array}$ & 32 & 15 & 2 & $20^{\circ}$ & 0 & - \\
\hline $\begin{array}{l}\text { Chen } \\
\text { und } \\
\text { Kollegen }\end{array}$ & 17 & 17,7 & - & 0 & 29,4 & $10^{\circ}$ bis $20^{\circ}$ \\
\hline
\end{tabular}

Tabelle 24: Ergebnisse des Streckdefizits im PIP- und DIP-Gelenk nach der Rekonstruktion von Fingerkuppenverletzungen mittels neurovaskulärer Insel-Lappenplastik

Die insgesamt gute aktive Beweglichkeit in unserer Studie führen wir auf die Tatsache zurück, dass es sich um Langzeit-Ergebnisse handelt und die Möglichkeit einer Verbesserung des Streckdefizites sowie der TAM über einen längeren Zeitraum besteht. Dafür spricht auch

Diskussion 
eine Steigerung der TAM in der Studie von Usami ${ }^{160}$ von $90,9 \%$ auf $92,3 \%$ nach einer Nachbeobachtungszeit von 6 im Vergleich zu 12 Monaten.

Eine obligatorische Schiene nach der Operation bleibt umstritten. Da unsere Ergebnisse denen anderer Studien, welche standardisiert eine Schiene verwenden, nicht unterliegen, ist eine Schiene nach jeder Operation möglicherweise nicht erforderlich. In der Studie von Varitimidis und Dailiana ${ }^{72}$ wurden standardisiert die Extensionsübungen und bei weiterhin bestehenden Streckdefiziten eine spezielle Streckschiene für die Nacht verwendet.

In einer anderen Studie von Kayalar ${ }^{156}$ wurde die Immobilisierung mittels Gipsschiene eine Woche nach der Operation angewendet. Anschließend fand im Fall einer Kontraktur eine Nachbehandlung mittels Streckschiene statt.

Andere Lappenplastiken wie z.B. der Cross-Finger-Lappen erfordern zusätzlich zum verletzten Finger eine Ruhigstellung oder Bewegungseinschränkung des Spenderfingers für mindestens zwei Wochen und tragen ebenfalls das Risiko einer Bewegungseinschränkung in den Fingergelenken mit sich. Dies muss unter Umständen mit einer Physiotherapie oder auch Schienenbehandlung therapiert werden..$^{96,100,114}$

In der Studie von Aydin ${ }^{100}$ und Kollegen wurden nach einer Nachbeobachtungszeit von 12 Monaten insgesamt 25 Patienten mit 28 rekonstruierten Fingerkuppen untersucht. Davon wurden 18 Fingerkuppenverletzungen mittels dorso-lateraler neurovaskulärer homodigitaler Insel-Lappenplastik und 10 mittels Cross-Finger-Lappenplastik rekonstruiert. Es konnte eine statistisch signifikant bessere $\mathrm{ROM}^{\circ}$ um ca. $20^{\circ}$ in DIP- und PIP-Gelenken nach Rekonstruktion mittels Insel-Lappenplastik im Vergleich zu Cross-Finger-Lappenplastik nachgewiesen werden.

Die Rekonstruktion von Fingerkuppenverletzungen mit retrograden neurovaskulären InselLappen mit Neurorrhaphie kann die Entstehung einer Beugekontraktur minimieren, zeigt aber oft eine schlechtere Sensibilität als anterograde neurovaskuläre Insel-Lappen. ${ }^{173}$ Ein Beispiel dafür ist ein Vergleich von 6 retrograden mit 12 anterograden Insel-Lappenplastiken in der Studie von Aydin ${ }^{100}$ und Kollegen. Bei der Gruppe von retrograden Insel-Lappen war die ROM in PIP-Gelenk um $8^{\circ}$ und im DIP-Gelenk um $6^{\circ}$ besser als bei anterograden, dagegen lag die mittlere 2-PD bei anterograden Insel-Lappen bei 7,3 $\mathrm{mm}$ und bei retrograden bei $9 \mathrm{~mm}$.

Diskussion

$-89-$ 


\subsection{Kraft}

Der Mittelwert der Fingerkraft im Schlüsselgriff mit Hilfe des Pinch-Gauge zwischen Daumen und den vier Fingerspitzen im Wechsel wurde bei fast allen Messungen an den gesunden Fingern gering größer gemessen ohne eine statistisch signifikante Differenz. Das veranschaulicht einen nur geringen Kraftverlust am betroffenen Finger.

In der Studie von Varitimidis und Dailiana ${ }^{72}$ wurden 63 Fingerkuppenverletzungen durch neurovaskuläre Insel-Lappenplastiken rekonstruiert und durchschnittlich nach 46 Monaten nachuntersucht. Hier lag der mittlere Wert der Fingerkraft bei 5,4 kg im Normbereich. Die Messungen wurden nicht mit dem gesunden Finger verglichen.

Der Mittelwert von drei Kraftmessungen der Hand bei unseren Patienten mittels JamarDynamometer zeigte einen geringen Kraftverlust an der betroffenen Hand um ca. 8,8 \%. Dies könnte durch ein schonendes Verhalten und ein geringeres Einsetzen der Hand mit operierten Fingern erklärt werden. Bei anderen vergleichbar großen Studien wurde häufig keine Kraft im Handgelenk gemessen. ${ }^{15,150,152,153,156}$ Ebenfalls werden die Ergebnisse der Kraftmessung mit Pinch-Gauge oder Jamar-Dynamometer nach der Rekonstruktion von Fingerkuppenverletzungen mittels Folientherapie nicht dargestellt. ${ }^{52,53,55,163}$

\subsection{Sensibilität}

Die Wiederherstellung einer sensiblen Fingerkuppe im Sinne eines Tastsinnorgans stellt eine große Herausforderung für den behandelnden Chirurg dar. ${ }^{174}$

In unserer Studie lag die mittlere 2-PD bei 5,1 mm, mit einer Spannweite von 2 bis $8 \mathrm{~mm}$. Dies spricht laut der Richtlinie von „Modified American Society for Surgery of the Hand“ zur Bewertung der 2-PD ${ }^{175}$ für ein ,exzellentes Ergebnis“.

Zusätzlich beurteilen wir Sensibilitätsdefizite mittels SWMT, was bei großen Patientengruppen in anderen Nachuntersuchungsstudien noch aussteht. ${ }^{72,115,152,158}$

Der SWMT ergab bei 59,3 \% eine normale Sensibilität. Bei allen Patienten war die Sensibilität besser als 4,31N.

Ähnliche Ergebnisse zeigen sich in der Studie von Kayalar ${ }^{156}$ und Kollegen mit 115 durch neurovaskuläre Insel-Lappen rekonstruierten Fingerkuppen mit einer mittleren Diskussion $-90-$ 
Nachuntersuchungsdauer von 41 Monaten. Hier lag der SWM Test in 87,3\% bei 4,31N oder besser. Die 2-PD lag bei 66,4 \% der Fingerkuppen zwischen 2 und 4 mm, allerdings mit einer deutlichen Spannweite bei allen Patienten von 2 bis $11 \mathrm{~mm}$.

Die publizierte Studie von Varitimidis und Dailiana ${ }^{72}$ mit 63 Fingerkuppenverletzungen und einer mittleren Nachbeobachtungzeit von 46 Monaten konnte gute Ergebnisse der 2-PD mit einem mittleren Wert von $4 \mathrm{~mm}$ in 93,7\% und einer Spannweite von 3 bis $6 \mathrm{~mm}$ nach der Rekonstruktion mittels neurovaskulärer Insel-Lappenplastik zeigen.

Bei einer anderen Studie von Lok und Kollegen ${ }^{155}$ bei 11 Patienten mit einer mittleren Nachbeobachtungzeit von 3,7 Monaten lag die 2-PD in 90,9\% unter $6 \mathrm{~mm}$.

In der Arbeit von Borman ${ }^{158}$ und Kollegen bei insgesamt 27 anterograden homodigitalen neurovaskulären Insel-Lappenplastiken mit einer mittleren Nachbeobachtungszeit von 4,2 Monaten konnten ähnlich gute Ergebnisse der 2-PD gezeigt werden. Hier lag der mittlere Wert bei $4,3 \mathrm{~mm}$ mit einer Spannweite von 3 bis $8 \mathrm{~mm}$.

Es zeigt sich, dass sowohl bei unserer Patientengruppe als auch bei anderen Studien insgesamt eine sehr gute Sensibilität nach der Rekonstruktion von Fingerkuppenverletzungen mittels neurovaskulären Insel-Lappen erzielt wird. Dies könnte darauf zurückzuführen sein, dass bei dieser Methode ein Prinzip der Rekonstruktion „gleiches mit gleichem“ verwendet wird. Han ${ }^{176}$ und Kollegen analysieren die Sensibilität zwischen Lappen mit und ohne Anschluss eines sensiblen Nervs. Insgesamt wurden 44 Fingerkuppen nach ca. 6 Monaten nachuntersucht. Die mittlere 2-PD lag bei Lappen mit Innervation bei 6,2 $\mathrm{mm}$ und ohne Innervation bei 10,2 mm. Wenn die neurovaskulären Insel-Lappen für die Fingerkuppenrekonstruktion nicht von einer sensiblen Fingerkuppe bzw. „ähnlichem“ Gewebe genommen wird ist die Sensibilität nicht immer ausreichend.

Ein Beispiel dafür ist die Studie von Tsai ${ }^{150}$ und Kollegen mit einer mittleren Nachbeobachtungsdauer von 62 Monaten und einer Rekonstruktion von 16 schrägverlaufenden Fingerkuppendefekten. Die neurovaskulären Insel-Lappen wurden 5 mm lateral und proximal des Nagelbettes an der dorsalen Seite der Finger entnommen. Hier war die 2-PD bei 43,7 \% der Patienten schlechter als $6 \mathrm{~mm}$ und bei einem Patienten sogar über $15 \mathrm{~mm}$. In der Arbeit von Aydin ${ }^{100}$ und Kollegen bei 18 Fingerkuppenverletzungen mit einer mittleren Nachbeobachtungzeit von 12 Monaten, welche durch eine dorso-laterale Diskussion - 91 - 
neurovaskuläre homodigitale Insel-Lappen versorgt wurden, lag die mittlere 2-PD bei 7,8 $\mathrm{mm}$. Dabei weisen die anterograden Insel-Lappen mit 7,3 $\mathrm{mm}$ eine bessere 2-PD im Vergleich zur retrograden mit $9 \mathrm{~mm}$.

In einer umfangreichen Studie von Kleinert ${ }^{94}$ und Kollegen wurden bei insgesamt 56 Patienten im Alter von 1 bis 67 Jahren, ohne Angabe der Nachbeobachtungszeit, die Ergebnisse nach der Rekonstruktion der Fingerkuppenverletzungen mit Cross-FingerLappenplastik analysiert. Insgesamt wurde die 2-PD bei 22 der Patienten (39,2 \%) schlechter als $6 \mathrm{~mm}$ gemessen. In der Arbeit von Aydin ${ }^{100}$ und Kollegen bei 9 Patienten und 10 Fingerkuppenverletzungen mit einer mittleren Nachbeobachtungzeit von 12 Monaten, welche durch Cross-Finger-Lappenplastik versorgt wurden, lag die mittlere 2-PD bei 10,7 $\mathrm{mm}$.

Die insgesamt schlechte Sensibilität der Fingerkuppen nach Cross-Finger-Lappenplastik im Vergleich zur neurovaskulären Insel-Lappenplastik könnte darauf zurückzuführen sein, dass ein Vollhauttransplantat bei einer Cross-Finger-Lappenplastik aus der volaren Seite der Mittelphalanx stammt und wenige Nervenendigungen, im Vergleicht zur Fingerspitze, enthält. Hier wird der Fingerkuppendefekt nicht mit ähnlichem Gewebe rekonstruiert.

Die konservative Semiokklusionstherapie ist eine neue Alternative einer operativen Behandlung. ${ }^{51}$ Es handelt sich um eine schnelle funktionelle Therapieoption ohne Notwendigkeit einer Ruhigstellung oder Morbidität von anderen Fingern. Vor allem ist diese Methode durch seine exzellente Wiederherstellung von Sensibilität, wie in der Tabelle 25 dargestellt, bekannt. Alle diese Studien beschreiben Fingerkuppenverletzungen von Zone I bis III nach Allen-Klassifikation. Die durchschnittliche 2-PD liegt bei 2,5 bis 5,8 mm mit einer Spannweite von 2 bis $8 \mathrm{~mm}$.

Diskussion

- 92 - 


\begin{tabular}{|c|c|c|c|c|c|}
\hline Studie & 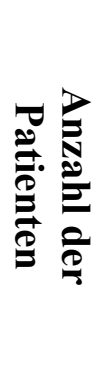 & 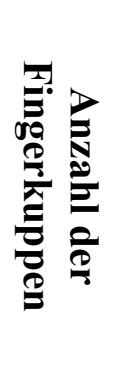 & 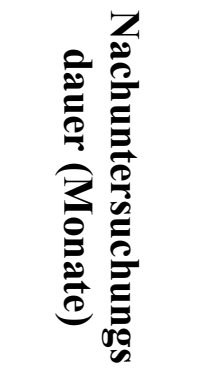 & 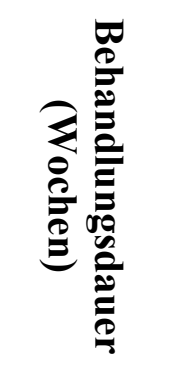 & 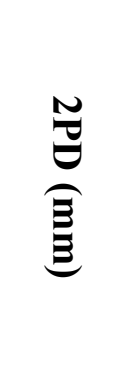 \\
\hline $\begin{array}{l}\text { Mennen und } \\
\text { Wiese } 51\end{array}$ & 200 & 200 & 3 & 3 bis 4,2 & 2,5 \\
\hline $\begin{array}{l}\text { Quell }{ }^{53} \text { und } \\
\text { Kollegen }\end{array}$ & 42 & 42 & 2 bis 6 & 2 bis 6 & 2 bis 8 \\
\hline $\begin{array}{c}\text { Hoigné }{ }^{52} \text { und } \\
\text { Kollegen }\end{array}$ & 17 & 19 & 6 bis 12 & 6,5 & 4,3 \\
\hline $\begin{array}{l}\text { Dereskewitz }^{163} \\
\text { und Kollegen }\end{array}$ & 100 & 100 & - & 3 & 5,8 \\
\hline Eigene Studie & 28 & 29 & 42 bis 169 & 0 bis 2 & 2 bis 8 \\
\hline
\end{tabular}

Tabelle 25: Vergleich der 2-PD nach der Rekonstruktion der Fingerkuppenverletzungen mittels Semiokklusionstherapie und Ergebnissen der eigenen Studie

Eine neue Studie von Quadlbauer ${ }^{55}$ und Kollegen beschreibt die Ergebnisse von Fingerkuppenverletzungen mittels Okklusionsverband von Zone III (9 Patienten) und Zone IV (4 Patienten) nach Allen-Klassifikation. Die mittlere Nachbeobachtungsdauer lag bei 15 (Zone III) und 27 Monaten (Zone IV). In 77 \% wurde eine normale Sensibilität mittels SWMT ohne genaue Angaben der 2PD beschrieben.

Dereskewitz ${ }^{163}$ und Kollegen veröffentlichen eine prospektive Fallstudie ohne Angaben der Nachuntersuchungsdauer über 100 überwiegend männlichen Patienten mit Fingerkuppenverletzungen, davon $77 \%$ mit Beteiligung von Knochen. Alle Patienten wurden mittels Folienverband therapiert. Der mittlere Wert der 2-PD lag bei 5,8 $\mathrm{mm}$. Allerdings weisen die Fingerkuppenverletzungen mit freiliegenden Knochen eine schlechtere 2-PD von 6,7 bis 7,8 mm auf. Auch die Regeneration der Weichteilgewebe lag bei diesen Patienten bei nur $70 \%$.

Sowohl eine operative Versorgung mittels neurovaskulären Insel-Lappen als auch ein konservativer Semiokklusionsverband erreichen eine Wiederherstellung der Sensibilität 
annährend dem physiologischen Niveau, möglicherweise korreliert das Ergebnis mit der Schwere der Verletzung.

\subsection{Komplikationen}

Folgende Frühkomplikationen wie Nekrose, Infektion oder Blutung traten bei unserer Patientengruppe in 9,4\% auf. Keiner der nachuntersuchten Patienten wies Neurombeschwerden auf. In der Arbeit von Foucher ${ }^{115}$ und Kollegen wurde insgesamt bei $11 \%$ der neurovaskulären Insel-Lappen nach einer Nachbeobachtungszeit von 15 bis 72 Monaten eine Nekrose oder Verlust beschrieben. In einer anderen Langzeitstudie, mit einer mittleren Nachbeobachtungszeit von 126 Monaten, von Cook $^{157}$ und Kollegen trat die partielle Nekrose lediglich bei einem (5,5\%) von 18 neurovaskulären Insel-Lappen mit einer darauffolgender Revision auf. Lanzetta ${ }^{152}$ und Kollegen berichten in ihrer Studie, mit einer mittleren Nachbeobachtungszeit von 30 Monaten, über eine totale Nekrose der neurovaskulären Insel-Lappen bei einem (4\%) von 25 Patienten. Bei keinem von diesen rekonstruierten Fingerkuppen wurde eine Neurombildung dokumentiert und es konnte eine gute Weichteildeckung erzielt werden. In den meisten Studien traten keine Frühkomplikationen, Verlust der neurovaskulären Insel-Lappen, Neurombildung oder Morbidität der Spenderstelle nach der Fingerkuppen Rekonstruktion auf. 72,150,155,160,172

Bei konservativer Therapie mittels Okklusionsverband wurden Frühkomplikationen ${ }^{51,52,163}$ selten und meistens bei Fingerkuppenverletzungen mit freiliegenden Knochen beobachtet. Quell $^{53}$ und Kollegen beschreiben bei insgesamt zwei $(16,7 \%)$ von 12 Fingerkuppenverletzungen mit Knochenbeteiligung eine Wundheilungsstörung, bei einem $(8,4 \%)$ chronische Osteitis und bei einem ein Narbenneurom.

In der Nachuntersuchungsstudie von Hoigné ${ }^{52}$ und Kollegen kam es bei 17 Patienten und 19 Fingerkuppenamputationen mit Knochenbeteiligung in einzelnen Fällen zur Sensibilitätsstörung und Neurombildung ohne die Notwendigkeit einer operativen Behandlung.

Die Tabelle 26 gibt einen Überblick von aufgetretenen Komplikationen wie Kälteempfindlichkeit, objektives Hoffmann-Tinel-Zeichen und Taubheitsgefühl bzw. 
Druckschmerz im Bereich der Fingerkuppe nach der Fingerkuppenrekonstruktion mittels neurovaskulärer Insel-Lappenplastik im Vergleich andere Studien mit unserer.

\begin{tabular}{|c|c|c|c|c|c|}
\hline Studie & 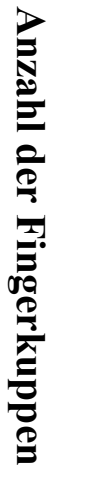 & 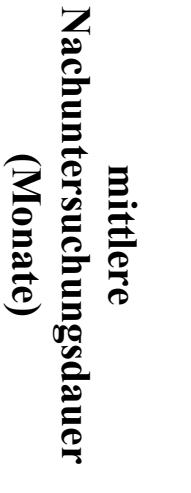 & 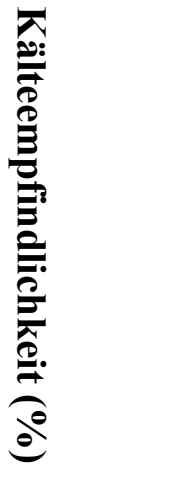 & 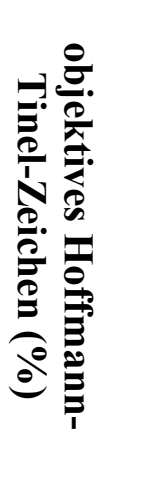 & 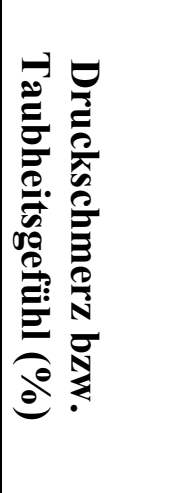 \\
\hline Eigene Studie & 29 & 105 & 48,3 & 24,1 & 17,2 \\
\hline Tsai ${ }^{150}$ und Kollegen & 16 & 62 & 68,7 & 43,8 & 56,3 \\
\hline Usami $^{160}$ und Kollegen & 17 & 12,5 & - & 23,5 & 17,6 \\
\hline $\begin{array}{c}\text { Foucher }^{115} \text { und } \\
\text { Kollegen }\end{array}$ & 64 & 15 bis 72 & $\begin{array}{c}\text { Mehrheit } \\
\text { der } \\
\text { Patienten }\end{array}$ & - & - \\
\hline Cook $^{157}$ und Kollegen & 18 & 126 & 29,4 & - & - \\
\hline $\begin{array}{c}\text { Varitimidis und } \\
\text { Dailiana }^{72}\end{array}$ & 63 & 46 & 6,4 & - & - \\
\hline $\begin{array}{c}\text { Kayalari }^{156} \text { und } \\
\text { Kollegen }\end{array}$ & 115 & 41 & 48,9 & - & - \\
\hline Adani ${ }^{171}$ und Kollegen & 32 & 15 & 28 & - & - \\
\hline
\end{tabular}

Tabelle 26: Langzeitkomplikationen andere Studien und unserer

Die meisten Studien und die Ergebnisse unserer Arbeit zeigen eine relativ hohe Kälteempfindlichkeit bzw. Kälteintoleranz verschiedener Intensität unabhängig von der Nachbeobachtungsdauer.

Eine Ausnahme stellt die Folienverbandstherapie, unter deren Anwendung tritt, in der Mehrheit der Fälle, keine Kälteempfindlichkeit auf..$^{51,52,163}$ In der Studie von Quell ${ }^{53}$ und Kollegen wurde bei insgesamt 52 rekonstruierten Fingerkuppen nach Behandlungsende eine Schmerzfreiheit und keine Kälteintoleranz beschrieben. Die Autoren merken an, dass jedoch die Kälteintoleranz bei warmem Wetter bei fast allen Patienten auftrat. Weitere Langzeitergebnisse wurden nicht berichtet.

Diskussion 


\subsection{Nagelwachstumstörungen}

Die häufig in der Literatur beschriebene Nagelwachstumstörung in Form eines Krallennagels nach einer operativen Behandlung wurde auch in unserer Patientengruppe bei 63,6\% beobachtet. In der Studie von Varitimidis $^{72}$ und Kollegen mit einer mittleren Nachbeobachtungszeit von 41 Monaten wurde eine Bildung des Krallennagels bei insgesamt $7 \%$ der Fingerkuppen, welche durch neurovaskuläre Insel-Lappenplastik versorgt wurden, beschrieben. Die gleiche Anzahl wurde in der Arbeit von Kayalar ${ }^{156}$ und Kollegen nach einer mittleren Nachbeobachtungsdauer von 41 Monaten dokumentiert.

In einer prospektiven Fallstudie von Dereskewitz ${ }^{163}$ und Kollegen bei 100 Fingerkuppenverletzungen, welche durch Folienverband versorgt wurden, traten Nagelwachstumsstörungen bei insgesamt $18 \%$ der Patienten auf. In der Studie von Hoigné52 und Kollegen kam bei einem von 19 Fingerkuppen (5,3 \%), welche mittels Okklusionstherapie behandelt wurden, nach einer Nachuntersuchungsdauer von 6 bis 12 Monaten zur Beeinträchtigung des Nagelwachstums.

Die Langzeitergebnisse einer Spiral-Lappenplastik von $\operatorname{Lim}^{30}$ weisen auf eine Nageldeformation hin, welche sich nach einer chirurgischen Rekonstruktion mit der Zeit verschlechtern kann. Daraus ergibt sich das Erfordernis einer präoperativen Aufklärung über eine mögliche Nagelwachstumsstörung nach der Rekonstruktion.

Trotz der hohen Anzahl von Krallennagelbildungen bei unseren Patienten und einer Differenz der Nagellänge bzw. Form waren alle Patienten mit dem Erhalt des Nagels zufrieden und haben dies nicht als störend empfunden. Sie haben gelernt frühzeitig den Nagel zu schneiden, wodurch es zu keinen Schmerzen im Nagelbett oder Hängenbleiben kommt.

\subsection{Limitierung}

Unsere Studie unterliegt einigen Limitationen. Es handelt sich um eine monozentrische Studie. Die Nachuntersuchung erfolgte von insgesamt 28 Patienten mit 29 Fingerkuppenverletzungen, daher ist dies eine kleine Stichprobe. Es gibt keine Information über die Richtung der Verletzung oder die Form des Defektes, da die Klassifikation der Fingerkuppenverletzungen nach Allen ${ }^{31}$ angenommen wurde. Die Verwendung von komplexen Klassifikationen mit mehr Unterteilung wäre genauer, ${ }^{177}$ aber diese wurde von Diskussion - 96 - 
einigen Autoren als zu kompliziert angesehen ${ }^{72}$ und daher auch in anderen Studien häufig nicht verwendet.

Die Analyse der postoperativen Ergebnisse konnte durch eine genaue Fotodokumentation und Ausmessung der Fingerkuppenverletzungen vor der Operation sowie die Durchführung der Operation durch den gleichen Operateur verbessert werden.

Als eine weiterführende Studie kann ein direkter Vergleich der Rekonstruktion von Fingerkuppenverletzungen zwischen neurovaskulären Insel-Lappen und Folientherapie oder zwischen verschiedenen Lappenplastiken untereinander erfolgen. Dafür fehlen homogene Studien von Fingerkuppenrekonstruktionen mittels Semiokklusionstherapie bei Verletzungen nach Allen-Klassifikation Zone III und IV.

\subsection{Schlussfolgerung}

Eine sehr hohe Patientenzufriedenheit und eine gute Langzeitfunktion können nach der Rekonstruktion der Fingerkuppenverletzungen mit anterograden homodigitalen neurovaskulären Insel-Lappen erreicht und in dieser Studie bewiesen werden. Es handelt sich um eine Ein-Schritt-Operation mit sofortiger Wiederherstellung von Sensibilität der Fingerkuppe ohne die Notwendigkeit einer Morbidität am benachbarten Finger. Eine obligatorische Schienung oder Z-Plastik, um einer Beugekontraktur im PIP-Gelenk entgegenzuwirken, ist möglicherweise nicht erforderlich. Bei den meisten Patienten konnten eine normale Fingerlänge sowie Beweglichkeit erzielt werden. Die weiteren Studien sind erforderlich, um die Langzeitergebnisse verschiedener therapeutischer Optionen zu vergleichen.

Diskussion

$-97-$ 


\section{Zusammenfassung}

Verletzungen der Fingerkuppen stellen einen häufigen Grund für die Vorstellung in der

Notaufnahme dar. Während viele Verletzungen konservativ behandelt werden können, benötigen einige Patienten eine operative Versorgung. Dabei kommen verschiedene operative Verfahren zur Anwendung, darunter eine Fingerkuppenrekonstruktion mit einer neurovaskulären Insel-Lappenplastik. ${ }^{1-3}$

Ziel der neurovaskulären Insel-Lappenplastik ist die Wiederherstellung einer taktil sensiblen und wieder belastungsfähigen Fingerkuppe ohne ein Längendefizit des Fingers.

In der vorliegenden Studie wurden Langzeit-Behandlungsergebnisse mit einer mittleren Nachuntersuchungsdauer von 105 Monaten bei 28 Patienten mit 29 durch neurovaskuläre Insel-Lappenplastiken rekonstruierten Fingerkuppen in der Berufsgenossenschaftlichen Unfallklinik Frankfurt am Main erfasst. Die untersuchten Patienten hatten zum Zeitpunkt der Verletzung ein Durchschnittsalter von 38,4 Jahren. Es handelte sich überwiegend um männliche und berufstätige Patienten.

Es wurden nur Fingerkuppenverletzungen mit freiliegenden Knochen (Allen-Klassifikation Zone III und IV) operativ versorgt. In unserer Studie traten am häufigsten die Verletzungen am Mittelfinger, Zeigefinger und Ringfinger auf. Die Mehrheit der Fingerkuppenverletzungen geschah in Folge eines Arbeitsunfalls, die Arbeitsunfähigkeitsdauer betrug ca. 6,1 Wochen. Die maximale Größe eines neurovaskulären Insel-Lappen lag bei 6 x 3,5 cm.

Alle Patienten waren mit den Behandlungsergebnissen anhand der numerischen Rating-Skala und des DASH Fragebogens bezüglich Funktionalität sowie dem ästhetischen Outcome zufrieden und würden sich wieder operieren lassen.

Die Sensibilität konnte anhand der Zwei-Punkte-Diskrimination sowie Semmes-Weinstein Monofilament-Testes als gut bewertet werden und normale physiologische Werte erreichen. Die Narbe war überwiegend weich und in der Mehrheit der Fälle entsprach sie anhand der Vancouver Scar Scale Werte annähend der normalen Haut. Zwei Drittel der Patienten gaben keine Schmerzen in Ruhe an. Die Hälfte der Patienten gaben Schmerzen unter Belastung anhand der numerischen Rating-Scala an. 
Trotz der hohen Anzahl von Krallennagelbildungen in 56,5 \% und einer Differenz der Nagellänge bzw. Form waren alle Patienten mit dem Erhalt des Nagels zufrieden und haben dies subjektiv nicht als störend empfunden.

Als besonders beeinträchtigend wurde eine Kälteempfindlichkeit von 48,3 \% Patienten beschrieben.

Der Mittelwert der Fingerkraft im Schlüsselgriff mit Hilfe des Pinch-Gauge zwischen Daumen und den vier Fingerspitzen im Wechsel wurde bei fast allen Messungen an den gesunden Fingern gering größer gemessen ohne eine statistisch signifikante Differenz. Die Messung der Handkraft mittels Jamar-Dynamometer ergab ein Defizit von 8,8 \% (Vergleich betroffene zur gesunden Hand).

Bei drei von 24 Patienten hat sich eine Beugekontraktur im Interphalangealgelenk von $5^{\circ}$, $15^{\circ}, 20^{\circ}$ und bei einem von 22 Patienten im distalen Interphalangealgelenk von $10^{\circ}$ gebildet. Zum Nachuntersuchungszeitpunkt wurden durch die Untersucherin ein Hoffmann-TinelZeichen in $24,1 \%$ und Druckschmerz in 17,2 \% im Bereich der verletzten Fingerkuppe festgestellt. Subjektiv empfand kein Patient diese Symptome als störend und alle berufstätigen Patienten konnten ihre vor dem Unfall ausgeübte Tätigkeit wieder aufnehmen. Diese Studie konnte belegen, dass die Defektdeckung der Fingerkuppenverletzungen mit Hilfe von neurovaskulären Insel-Lappenplastiken ein sehr gutes ästhetisches und funktionelles Ergebnis mit einer fast identischen Hautqualität erzielt. Mit dieser Methode konnte eine Wiederherstellung des Weichteilgewebes der sensiblen Fingerkuppe auch bei großflächigen Defekten der Fingerkuppe erreicht werden. Die subjektive Patientenzufriedenheit mit dieser Rekonstruktionsmethode ist hoch. 


\section{Abstract}

Fingertip injuries are a common reason for presentation at emergency department. While many injuries can be treated conservatively, some patients require a surgical treatment. Various surgical procedures can be applied, including fingertip reconstruction with neurovascular island-flap repair. ${ }^{1-3}$

The objective of neurovascular island-flap repair is to restore a fingertip such that it is sensitive to touch and is resilient, without causing a length deficit of the finger.

In the present study, long-term treatment results with a mean follow-up period of 105 months were recorded in 28 patients, in whom 29 finger tips were reconstructed using neurovascular island-flap repairs at the Berufsgenossenschaftliche Unfallklinik, Frankfurt am Main. The patients investigated had an average age of 38.4 years at the time of injury. They were predominantly male patients who were in employment.

Only fingertip injuries with exposed bone (Allen classification zone III and IV) were treated surgically. In our study, the most common injuries were to the second, third and fourth phalanges. The majority of fingertip injuries occurred as a result of an accident at work, and the duration of incapacity to work was approx. 6.1 weeks. The maximum size of a neurovascular island-flap was $6 \times 3.5 \mathrm{~cm}$.

All patients were satisfied with the treatment results based on the numerical rating scale and the DASH questionnaire in terms of functionality and aesthetic outcomes, and would be open to further surgery.

Sensitivity could be evaluated as good on the basis of the two-point discrimination and Semmes-Weinstein monofilament tests, with normal physiological values being achieved. The scar was predominantly soft, and in the majority of cases it corresponded to near-normal skin as per Vancouver Scar Scale values. Two-thirds of patients reported no pain at all at rest. Half of the patients reported pain under stress based on the numerical rating scale.

Despite the high number of nail deformations, occurring in $56.5 \%$, and a difference in nail length and shape, all patients were satisfied with the preservation of the nail and subjectively did not perceive this as an impairment.

Sensitivity to the cold in $48.3 \%$ of patients was described as a particular impairment. 
The average finger force during key pinch, measured using a pinch gauge between the thumb and the four finger tips in alternation, was measured as slightly greater in almost all measurements on the healthy fingers, with no statistically significant difference identified. Measurements of hand force using a Jamar dynamometer showed a deficit of $8.8 \%$ (comparison between the affected hand and healthy hand).

In 3 out of 24 patients, a flexing contracture was identified in the interphalangeal joint at $5^{\circ}$, $15^{\circ}$, and $20^{\circ}$ respectively; and in one out of 22 patients, the contracture was identified in the distal interphalangeal joint at $10^{\circ}$.

At the time of examination, the investigator recorded a Hoffmann-Tinel sign in $24.1 \%$, and pain on pressure in $17.2 \%$ when applied to the area of the injured fingertip. Subjectively, no patient found these symptoms to be an impairment. All patients in employment were able to resume the work they carried out as before the accident.

This study was able to demonstrate that covering the defect on fingertip injuries using neurovascular island-flap repair achieves a very good aesthetic and functional result with an almost identical skin quality. Using this method, it was possible to restore the soft tissue of the sensitive fingertip even in cases of large-area defects of the fingertip. Subjective patient satisfaction with this reconstructive method is high.

Abstract 


\section{Literaturverzeichnis}

1. Sorock GS, Lombardi DA, Hauser RB, Eisen EA, Herrick RF, Mittleman MA. Acute traumatic occupational hand injuries: type, location, and severity. I Occup Environ Med. 2002;44(4):345-351.

2. Lemmon JA, Janis JE, Rohrich RJ. Soft-tissue injuries of the fingertip: methods of evaluation and treatment. An algorithmic approach. Plast Reconstr Surg. 2008;122(3):105e-117e.

3. Chau N, Gauchard GC, Siegfried C, et al. Relationships of job, age, and life conditions with the causes and severity of occupational injuries in construction workers. Int Arch Occup Environ Health. 2004;77(1):60-66.

4. Fassler PR. Fingertip Injuries: Evaluation and Treatment. J Am Acad Orthop Surg. 1996;4(1):84-92.

5. Panattoni JB, De Ona IR, Ahmed MM. Reconstruction of fingertip injuries: surgical tips and avoiding complications. The Journal of hand surgery. 2015;40(5):1016-1024.

6. Dereskewitz C. Fingerkuppen und Nagelbettverletzungen. In: Sauerbier M, ed. Die Handchirurgie. 1. Aufl. ed. München: Urban \& Fischer; 2015:72-82.

7. Peterson SL, Peterson EL, Wheatley MJ. Management of fingertip amputations. The Journal of hand surgery. 2014;39(10):2093-2101.

8. Schmidt HM, Lanz U, Kohnle G. Chirurgische Anatomie der Hand. Thieme; 2003.

9. Shrewsbury M, Johnson RK. The fascia of the distal phalanx. J Bone Joint Surg Am. 1975;57(6):784-788.

10. Zook EG. Anatomy and physiology of the perionychium. Hand Clin. 1990;6(1):1-7.

11. Dautel G. Substanzverluste der Hand. In: Merle M, Dautel G, Rehart S, eds. Chirurgie der Hand - Der Notfall. Vol 2. Stuttgart Georg Thieme Verlag; 2012:120-185.

12. Murai M, Lau HK, Pereira BP, Pho RW. A cadaver study on volume and surface area of the fingertip. The Journal of hand surgery. 1997;22(5):935-941.

13. Wilson WF, Hueston JT. The intratendinous architecture of the tendons of flexor digitorum profundus and flexor pollicis longus. The Hand. 1973;5:33-38.

14. Schrewsbury MM, Kuczynski K. Flexor digitorum superficialis tendon in the fingers of the human hand. The Hand. 1974;6:121-133.

15. Frenz C, Fritsch H, Hoch J. Plastinationshistologische Untersuchungen zur Insertion der Pars terminalis der Aponeurosis dorsalis an den dreigliedrigen Fingern. Annals of Anatomy Anatomischer Anzeiger. 2000;182(1):69-73.

16. Tos $\mathrm{P}$, Titolo $\mathrm{P}$, Chirila NL, Catalano F, Artiaco S. Surgical treatment of acute fingernail injuries. J Orthop Traumatol. 2012;13(2):57-62.

17. Horstmann E. Bau ubd Struktur des menschlichen Nagels. Zellforsch. 1955;41:532-555.

18. Horstmann E. Die Haut. In: Bargmann W, ed. Handbuch der mikroskopischen Anatomie des Menschen. Vol 3. Berlin: Springer; 1957.

19. Chaudakshetrin P, Kumar VP, Satku K, Pho RW. The arteriovenous pattern of the distal digital segment. J Hand Surg Br. 1988;13(2):164-166.

20. Brunelli F, Brunelli G. Vascular anatomy of the distal phalanx. Pers commun. 1989.

21. Flint M. Some observations on the vascular supply of the nail bedand terminal segments of the finger. Brit J Plast Surg. 1956;8:186-195.

22. Dautel G, Merle M. The blood supply of digital nerves: a microanatomical study of superficial and deep palmar venous networks. J Hand Surg Br. 1992;17(6):632-637.

Literaturverzeichnis - 102 - 
23. Moss SH, Schwartz KS, von Drasek-Ascher G, Ogden LL, 2nd, Wheeler CS, Lister GD. Digital venous anatomy. The Journal of hand surgery. 1985;10(4):473-482.

24. Smith DO, Oura C, Kimura C, Toshimori K. Artery anatomy and tortuosity in the distal finger. The Journal of hand surgery. 1991;16(2):297-302.

25. Schünke $\mathrm{M}$, Schulte $\mathrm{E}$, Schumacher U. PROMETHEUS Allgemeine Anatomie und Bewegungssystem. Vol 4. Stuttgart: Georg Thieme Verlag; 2014.

26. Chow S. Digital nerves in the terminal region of the thumb. The Hand. 1900;12(2):193-196.

27. Zenn MR, Hoffman L, Latrenta G, Hotchkiss R. Variations in digital nerve anatomy. The Journal of hand surgery. 1992;17(6):1033-1036.

28. Wood Jones F. The principles of anatomy as seen in the hand. Vol 2. London: Tindall \& Cox; 1944.

29. Speckmann E-J, Hescheler Jr, Köhling Rd. Physiologie. 6. Auflage ed. München: Urban \& Fischer; 2013.

30. Lim GJ, Yam AK, Lee JY, Lam-Chuan T. The spiral flap for fingertip resurfacing: short-term and long-term results. The Journal of hand surgery. 2008;33(3):340-347.

31. Allen MJ. Conservative management of finger tip injuries in adults. Hand. 1980;12(3):257265.

32. Rosenthal EA. Treatment of fingertip and nail bed injuries. Orthop Clin North Am. 1983;14(4):675-697.

33. Tamai S. Twenty years' experience of limb replantation--review of 293 upper extremity replants. The Journal of hand surgery. 1982;7(6):549-556.

34. Foucher G, Henderson H, Maneaud M, Merle M, Braun F. Distal digital replantation: One of the best indications for microsurgery. Int J Microsurg. 1981;3:263-270.

35. Ishikawa A, Ogawa Y, Soeda H, Yoshida Y. A new classification of the amputation level for the distal part of the fingers. J Jpn Soc Microsurg. 1990;3:54-62.

36. Hirase Y. Salvage of fingertip amputated at nail level: New surgical principles and treatment. Ann Plast Surg. 1997;38:151-157.

37. Evans DM, Bernardis C. A new classification for fingertip injuries. J Hand Surg Br. 2000;25(1):58-60.

38. Germann G, Rudolf KD, Levin SL, Hrabowski M. Fingertip and Thumb Tip Wounds: Changing Algorithms for Sensation, Aesthetics, and Function. The Journal of hand surgery. 2017;42(4):274-284.

39. Sauerbier $M$, Unglaub F. Perforator flaps in the upper extremity. Clin Plast Surg. 2010;37(4):667-676, vii.

40. Majno G. The Healing Hand. Cambridge: Harvard university press; 1975.

41. Gottlieb LJ, Krieger LM. From the reconstructive ladder to the reconstructive elevator. Plast Reconstr Surg. 1994;93(7):1503-1504.

42. Germann G, Sauerbier M, Rudolf KD, Hrabowski M. Management of thumb tip injuries. The Journal of hand surgery. 2015;40(3):614-622; quiz 623.

43. Braun M, Horton RC, Snelling CF. Fingertip amputation: review of 100 digits. 1985.

44. Stevenson TR. Fingertip and nailbed injuries. Orthop Clin North Am. 1992;23(1):149-159.

45. Buckley SC, Scott S, Das K. Late review of the use of silver sulphadiazine dressings for the treatment of fingertip injuries. Injury. 2000;31:301.

46. Aydin U, Ozbek S, Akin S, Ozyurtlu M. Custom subatmospheric dressing for fingertip injuries. Tech Hand Up Exrtem Surg. 2011;15(2):104-105.

Literaturverzeichnis - $103-$ 
47. Lee L, Lau P, Chan C. A simple and efficient treatment for fingertip injuries. J Hand Surg (Br). 1995;20:63.

48. Bossley CJ. Conservative treatment of digit amputations. N Z Med J. 1975;82(553):379-380.

49. Mühldorfer-Fodor M, Hohendorff B, Vorderwinkler KP, van Schoonhoven J, Prommersberger KJ. Behandlung von Fingerkuppendefektverletzungen mit dem Semiokklusionsverband nach Mennen und Wiese. Oper Orthop Traumatol. 2013;25(1):104114.

50. Dereskewitz C. Fingerkuppenverletzungen und Okklusionsverbände. Handchirurgie Scan. 2013;02(04):301-312.

51. Mennen $U$, Wiese A. Fingertip injuries management with semi-occlusive dressing. $J$ Hand Surg Br. 1993;18(4):416-422.

52. Hoigne $D$, Hug $U$, Schurch $M$, Meoli $M$, von Wartburg U. Semi-occlusive dressing for the treatment of fingertip amputations with exposed bone: quantity and quality of soft-tissue regeneration. J Hand Surg Eur Vol. 2014;39(5):505-509.

53. Quell M, Neubauer T, Wagner M. Die Behandlung von Fingerkuppendefektverletzungen mit einem semiokklusiven Verband. Handchir Mikrochir Plast Chir. 1998;30(1):24-29.

54. Krauss EM, Lalonde DH. Secondary healing of fingertip amputations: a review. Hand ( $N$ Y). 2014;9(3):282-288.

55. Quadlbauer S, Pezzei C, Jurkowitsch J, et al. Der Okklusionsverband zur Behandlung von Allen III und IV Fingerkuppenverletzungen als Alternative zu lokalen Lappenplastiken. Unfallchirurg. 2017;120(11):961-968.

56. Richter M. Fingerkuppendefekte: Ersetzt der Semiokklusivverband die Lappenplastik? Obere Extremität. 2010;1:6-13.

57. Martin C, Gonzalez del Pino J. Controversies in the treatment of fingertip amputations. Conservative versus surgical reconstruction. Clin Orthop Relat Res. 1998(353):63-73.

58. Winter GD. Healing of skin wounds and the influence of dressings on the repair progress. In: Harkiss KJ ed. Surgical Dressings and Wound Healing. Brandford: Brandford University Press; 1971:46-60.

59. Alvarez OM, Mertz PM, Eaglstein WH. The effect of occlusive dressings on collagen synthesis and re-epithelialization in superficial wounds. J Surg Res. 1983;35(2):142-148.

60. Hutchinson JJ, McGuckin M. Occlusive dressings: a microbiologic and clinical review. Am J Infect Control. 1990;18(4):257-268.

61. Buchan IA, Andrews JK, Lang SM. Clinical and laboratory investigation of the composition and properties of human skin wound exsudate under semi-permeable dressings. Burns. 1980;7(326-334).

62. Vogt PM, Lehnhardt M, Wagner D, Jansen V, Krieg M, Steinau HU. Determination of endogenous growth factors in human wound fluid: temporal presence and profiles of secretion. Plast Reconstr Surg. 1998;102(1):117-123.

63. Madden MR, Nolan E, Finkelstein JL, et al. Comparison of an occlusive and a semi-occlusive dressing and the effect of the wound exudate upon keratinocyte proliferation. $J$ Trauma. 1989;29(7):924-930; discussion 930-921.

64. De Boer P, Collison PO. The use of silver sulphadiazine occlusive dressings for finger-tip injuries. J Bone Joint Surg Br. 1981;63(B):545-547.

65. Damert HG, Altmann S. Behandlung von Fingerkuppenamputationen mittels Semiokklusivverband. 2012;115(9):798-801.

66. Zachary SV, Peimer CA. Salvaging the "unsalvageable" digit. Hand Clin. 1997;13(2):239-249.

Literaturverzeichnis

- 104 - 
67. Patten H. Split skin graft from the hypothenar area for fingertip avulsion. Plast Reconstr Surg. 1974;43:426.

68. Holm A, Zachariae L. Fingertip lesions. An evaluation of conservative treatment versus free skin grafting. Acta Orthop Scand. 1974;45(3):382-392.

69. Sturman M, Duran R. The lare results of fingertip injuries. J Bone Joint Surg (Am). 1963;45:289.

70. Clifford RH. Evaluation of three methods for finger tip injuries. Arch Surg. 1952;7:464-466.

71. Moynihan FJ. Long-term results of split-skin grafting in finger-tip injuries. $\mathrm{Br} \mathrm{Med} \mathrm{J}$. 1961;2(5255):802-806.

72. Varitimidis SE, Dailiana ZH, Zibis AH, Hantes M, Bargiotas K, Malizos KN. Restoration of function and sensitivity utilizing a homodigital neurovascular island flap after amputation injuries of the fingertip. J Hand Surg Br. 2005;30(4):338-342.

73. Gharb BB, Rampazzo A, Armijo BS, et al. Tranquilli-Leali or Atasoy flap: an anatomical cadaveric study. J Plast Reconstr Aesthet Surg. 2010;63(4):681-685.

74. Tranquilli-Leali E. Reconstructione dell apice falangi vaguali mediante autoplastica volare pedunculate per scorimento. Infort Traumatol Lavoro. 1935;1(1):93-186.

75. Atasoy E, loakimidis E, Kasdan ML, Kutz JE, Kleinert HE. Reconstruction of the amputated finger tip with a triangular volar flap. A new surgical procedure. J Bone Joint Surg Am. 1970;52(5):921-926.

76. Ma GF, Cheng JC, Chan KT, Chan KM, Leung PC. Finger tip injuries--a prospective study on seven methods of treatment on 200 cases. Ann Acad Med Singapore. 1982;11(2):207-213.

77. Takeishi M, Shinoda A, Sugiyama A, Ui K. Innervated reverse dorsal digital island flap for fingertip reconstruction. The Journal of hand surgery. 2006;31(7):1094-1099.

78. Frazier TG, Graham WP. Fingertip injuries. Pa Med. 1971;74:51-53.

79. Geissendörfer H. Beitrag zur Fingerkuppenplastik. Zentralb/ Chir. 1943;70:1107-1108.

80. Freiberg A, Manktelow R. The Kutler repair for fingertip amputations. Plast Reconstr Surg. 1972;50(4):371-375.

81. Foucher G, Delaere O, Citron N, Molderez A. Long-term outcome of neurovascular palmar advancement flaps for distal thumb injuries. Br J Plast Surg. 1999;52(1):64-68.

82. Moberg E. Aspects of Sensation in Reconstructive Surgery of the Upper Extremity. J Bone Joint Surg Am. 1964;46:817-825.

83. Kleinman WB, Strickland JW. Thumb reconstruction. In: Green DP, Hitchkiss R, Pederson WC, eds. Operative Hand Surgery. Vol 4th ed. New York: Churchill Livingstone; 1999:20682170.

84. Rohrich R, Antrobus S. Volar advancement flaps. In: Blair W, ed. Techniques in Hand Surgery. Baltimore: Williams and Wilkins; 1996:39-47.

85. O'Brien B. Neurovascular island pedicle flaps for terminal amputations and digital scars. $\mathrm{Br}$ J Plast Surg. 1968;21(3):258-261.

86. Germann G. Principles of flap design for surgery of the hand. Atlas Hand Clin. 1968;3:33-57.

87. Dellon AL. The extended palmar advancement flap. The Journal of hand surgery. 1983;8(2):190-194.

88. Venkataswami R, Subramanian N. Oblique triangular flap: a new method of repair for oblique amputations of the fingertip and thumb. Plast Reconstr Surg. 1980;66(2):296-300.

89. Saraf S, Tiwari V. Fingertip injuries. Indian J Orthop. 2007;41(2):163-168.

90. Hueston J. Local flap repair of fingertip injuries. Plast Reconstr Surg. 1966;37(4):349-350.

Literaturverzeichnis $\quad-105-$ 
91. Meyer-Marcotty MV, Kall S, Vogt PM. Neurovaskuläre Lappen zur Rekonstruktion von Fingerendglieddefekten. Unfallchirurg. 2007;110(5):433-446.

92. Gatewood J. A plastic repair of finger defects without hospitalization. J Amer Med ASS. 1927(87):1479.

93. Gurdin M, Pangman WJ. The repair of surface defects of fingers by trans-digital flaps. Plast Reconstr Surg (1946). 1950;5(4):368-371.

94. Kleinert HE, McAlister CG, MacDonald CJ, Kutz JE. A critical evaluation of cross finger flaps. J Trauma. 1974;14(9):756-763.

95. Megerle K, Palm-Broking K, Germann G. Der Crossfingerlappen. Oper Orthop Traumatol. 2008;20(2):97-102.

96. Wichelhaus A. Dorsale "Cross-finger"-Lappenplastik. Defektdeckung von palmaren Weichteilverletzungen der Finger. Unfallchirurg. 2015;118(2):170-172.

97. Battafarano RJ, Anderson RC, Meyers BF, et al. Perioperative complications after living donor lobectomy. J Thorac Cardiovasc Surg. 2000;120(5):909-915.

98. Nishikawa $\mathrm{H}$, Smith PJ. The recovery of sensation and function after cross-finger flaps for fingertip injury. J Hand Surg Br. 1992;17(1):102-107.

99. Lassner F, Becker M, Berger A, Pallua N. Sensory reconstruction of the fingertip using the bilaterally innervated sensory cross-finger flap. Plast Reconstr Surg. 2002;109(3):988-993.

100. Aydin HU, Savvidou C, Ozyurekoglu T. Comparison of Homodigital Dorsolateral Flap and Cross-Finger Flap for the Reconstruction of Pulp Defects. The Journal of hand surgery. 2018.

101. Littler JW. Neurovascular pedicle transfer of tissue in reconstructive surgeryof the hand. $J$ Bone Joint Surg Am. 1956;38:917-921.

102. Hilgenfeldt O. Operativer Daumenersatz. Enke, Stuttgart. 1950.

103. Holevich J. A New Method of Restoring Sensibility to the Thumb. J Bone Joint Surg Br. 1963;45:496-502.

104. Foucher $G$, Braun JB. A new island flap transfer from the dorsum of the index to the thumb. Plast Reconstr Surg. 1979;63(3):344-349.

105. Saalabian A, Rab M, van Schoonhoven J, Prommersberger KJ. [Foucher's first dorsal metacarpal artery island flap]. Oper Orthop Traumatol. 2009;21(6):614-619.

106. Elliot D. Specific flaps for the thumb. Tech Hand Up Extrem Surg. 2004;8(4):198-211.

107. Akyurek M, Safak T, Kecik A. Fingertip replantation at or distal to the nail base: use of the technique of artery-only anastomosis. Ann Plast Surg. 2001;46(6):605-612.

108. Kim WK, Lim JH, Han SK. Fingertip replantations: clinical evaluation of 135 digits. Plast Reconstr Surg. 1996;98(3):470-476.

109. Dautel G. Fingertip replantation in children. Hand Clin. 2000;16(4):541-546.

110. Yabe $\mathrm{T}$, Muraoka $\mathrm{M}$, Motomura $\mathrm{H}$, Ozawa T. Fingertip replantation using a single volar arteriovenous anastomosis and drainage with a transverse tip incision. The Journal of hand surgery. 2001;26(6):1120-1124.

111. Hahn HO, Jung SG. Results of replantation of amputated fingertips in 450 patients. $J$ Reconstr Microsurg. 2006;22(6):407-413.

112. Del Pinal F, Pennazzato D, Urrutia E. Primary Thumb Reconstruction in a Mutilated Hand. Hand Clin. 2016;32(4):519-531.

113. Del Pinal F, Moraleda E, de Piero GH, Ruas JS, Galindo C. Onycho-osteo-cutaneous defects of the thumb reconstructed by partial hallux transfer. The Journal of hand surgery. 2014;39(1):29-36. 
114. Katz RD. The anterograde homodigital neurovascular island flap. The Journal of hand surgery. 2013;38(6):1226-1233.

115. Foucher G, Smith D, Pempinello C, Braun FM, Citron N. Homodigital neurovascular island flaps for digital pulp loss. J Hand Surg Br. 1989;14(2):204-208.

116. Huang $\mathrm{Y}$, Liu $\mathrm{Y}$, Chen $\mathrm{T}$. Use of homodigital reverse island flaps for distal digital reconstruction. J Trauma. 2010;68(2):429-433.

117. Flint $M$, Harrison D. A local neurovascular island flap for fingertip reconstruction. Britisch Journal of Plastic Surgery. 1965;18:156-163.

118. Joshi BB. A local dorsolateral island flap for restoration of sensation after avulsion injury of fingertip pulp. Plast Reconstr Surg. 1974;54(2):175-182.

119. Segmuller G. Modification of the Kutler flap: neurovascular pedicle. Handchirurgie. 1976;8(2):75-76.

120. Biddulph SL. The neuro vascular flap in finger tip injuries. Hand. 1979;11(1):59-63.

121. Mouchet A, Gilbert A. Covering distal amputations of the finger using homodigital neurovascular island flaps. Ann Chir Main. 1982;1(2):180-182.

122. Schuind F, Van Genechten F, Denuit P, Merle M, Foucher G. Le lambeau en îlot homodactyle en chirurgie de la main. A propos de soixante cas. Annales de Chirurgie de la Main. 1985;4(4):306-309.

123. Lai CS, Lin SD, Yang CC. The reverse digital artery flap for fingertip reconstruction. Ann Plast Surg. 1989;22(6):495-500.

124. Kojima T, Tsuchida Y, Hirase Y, Endo T. Reverse vascular pedicle digital island flap. Br J Plast Surg. 1990;43(3):290-295.

125. Sullivan T, Smith J, Kermode J, Mclver E, Courtemanche DJ. Rating the burn scar. J Burn Care Rehabil. 1990;11(3):256-260.

126. Brusselaers N, Pirayesh A, Hoeksema H, Verbelen J, Blot S, Monstrey S. Burn scar assessment: a systematic review of different scar scales. J Surg Res. 2010;164(1):e115-123.

127. van der Wal MB, Verhaegen PD, Middelkoop E, van Zuijlen PP. A clinimetric overview of scar assessment scales. J Burn Care Res. 2012;33(2):e79-87.

128. Idriss N, Maibach HI. Scar assessment scales: a dermatologic overview. Skin Res Technol. 2009;15(1):1-5.

129. Briggs $M$, Closs JS. A descriptive study of the use of visual analogue scales and verbal rating scales for the assessment of postoperative pain in orthopedic patients. J Pain Symptom Manage. 1999;18(6):438-446.

130. Bijur PE, Latimer CT, Gallagher EJ. Validation of a verbally administered numerical rating scale of acute pain for use in the emergency department. Acad Emerg Med. 2003;10(4):390392.

131. Farrar JT, Young JP, Jr., LaMoreaux L, Werth JL, Poole RM. Clinical importance of changes in chronic pain intensity measured on an 11-point numerical pain rating scale. Pain. 2001;94(2):149-158.

132. Dixon JS. Agreement between horizontal and vertical visual analogue scales. Br J Rheumatol. 1986;25(4):415-416.

133. Hoffmann P, Buck-Gramcko D, Lubahn JD. The Hoffmann-Tinel sign. 1915. J Hand Surg Br. 1993;18(6):800-805.

134. Alfonso MI, Dzwierzynski W. Hoffman-Tinel sign. The realities. Phys Med Rehabil Clin N Am. 1998;9(4):721-736, v. 
135. Germann G, Wind G, Harth A. Der DASH-Fragebogen - Ein neues Instrument zur Beurteilung von Behandlungsergebnissen an der oberen Extremität. . Handchir Mikrochir Plast Chir. 1999;31(3):149-152.

136. Kleinert HE, Verdan C. Report of the Committee on Tendon Injuries (International Federation of Societies for Surgery of the Hand). The Journal of hand surgery. 1983;8(5 Pt 2):794-798.

137. Strickland JW, Glogovac SV. Digital function following flexor tendon repair in Zone II: A comparison of immobilization and controlled passive motion techniques. The Journal of hand surgery. 1980;5(6):537-543.

138. Strickland JW. Results of flexor tendon surgery in zone II. Hand Clin. 1985;1(1):167-179.

139. Kapandji A. Clinical test of apposition and counter-apposition of the thumb. Annales de chirurgie de la main. 1986;5(1):67-73.

140. Mathiowetz V, Weber K, Volland G, Kashman N. Reliability and validity of grip and pinch strength evaluations. The Journal of hand surgery. 1984;9(2):222-226.

141. Firrell JC, Crain GM. Which setting of the dynamometer provides maximal grip strength? The Journal of hand surgery. 1996;21(3):397-401.

142. Fess EE. Grip strenght. In: Clinical assessment recommendations. Vol 2nd ed. Chicago: American Society of Hand Therapists; 1992:41-45.

143. Akatsuka K, Noguchi Y, Harada T, Sadato N, Kakigi R. Neural codes for somatosensory twopoint discrimination in inferior parietal lobule: an fMRI study. Neuroimage. 2008;40(2):852858.

144. van Nes SI, Faber CG, Hamers RM, et al. Revising two-point discrimination assessment in normal aging and in patients with polyneuropathies. J Neurol Neurosurg Psychiatry. 2008;79(7):832-834.

145. Nolan MF. Two-point discrimination assessment in the upper limb in young adult men and women. Phys Ther. 1982;62(7):965-969.

146. Bigley GK. Sensation. In: rd, Walker HK, Hall WD, Hurst JW, eds. Clinical Methods: The History, Physical, and Laboratory Examinations. Boston1990.

147. Cope EB, Antony JH. Normal values for the two-point discrimination test. Pediatr Neurol. 1992;8(4):251-254.

148. Moberg E. Two-point discrimination test. A valuable part of hand surgical rehabilitation, e.g. in tetraplegia. Scand J Rehabil Med. 1990;22(3):127-134.

149. Semmes J, Weinstein S, Ghent L, Teuber H. Somatosensory changes after penetrating brain wounds in man. Cambridge, MA: Harvard University Press. 1960.

150. Tsai TM, Yuen JC. A neurovascular island flap for volar-oblique fingertip amputations. Analysis of long-term results. J Hand Surg Br. 1996;21(1):94-98.

151. Henry M, Stutz C. Homodigital Antegrade-Flow Neurovascular Pedicle Flaps for Sensate Reconstruction of Fingertip Amputation Injuries. The Journal of hand surgery. 2006;31(7):1220-1225.

152. Lanzetta M, Mastropasqua B, Chollet A, Brisebois N. Versatility of the homodigital triangular neurovascular island flap in fingertip reconstruction. J Hand Surg Br. 1995;20(6):824-829.

153. Chen CT, Wei FC. Lateral-dorsal neurovascular island flaps for pulp reconstruction. Ann Plast Surg. 2000;45(6):616-622.

154. Hamdi MF. Advancement fingertip homodigital neurovascular island flaps of long fingers. About 32 cases. Chir Main. 2011;30(2):105-109.

Literaturverzeichnis $\quad-108-$ 
155. Lok LW, Chan WL, Lau YK. Functional Outcomes of Antegrade Homodigital Neurovascular Island Flaps for Fingertip Amputation. J Hand Surg Asian Pac Vol. 2017;22(1):39-45.

156. Kayalar M, Bal E, Toros T, Sugun S, Ozaksar K, Gurbuz Y. The outcome of direct-flow neurovascular island flaps in pulp defects. Acta orthopaedica et traumatologica turcica. 2011;45:175-184.

157. Cook FW, Jakab E, Pollock MA. Local neurovascular island flap. The Journal of hand surgery. 1990;15(5):798-802.

158. Borman $\mathrm{H}$, Maral T, Tancer M. Fingertip reconstruction using two variations of direct-flow homodigital neurovascular island flaps. Ann Plast Surg. 2000;45(1):24-30.

159. Yuan F, McGlinn EP, Giladi AM, Chung KC. A Systematic Review of Outcomes after Revision Amputation for Treatment of Traumatic Finger Amputation. Plast Reconstr Surg. 2015;136(1):99-113.

160. Usami S, Kawahara S, Yamaguchi Y, Hirase T. Homodigital artery flap reconstruction for fingertip amputation: a comparative study of the oblique triangular neurovascular advancement flap and the reverse digital artery island flap. Journal of Hand Surgery (European Volume). 2013;40(3):291-297.

161. Zook EG. A study of nail bed injuries: causes treatment and prognosis. J Hand Surg. 1984;9(A):247-252.

162. Magerle K, Palm-Bröking K, Germann G. Der Crossfingerlappen. Oper Orthop Traumatol. 2008(2):97-102.

163. Dereskewitz C, Harling M, Nienhaus A, Partecke BD, Rudolf KD. Folientherapie von Fingerdefektverletzungen - Eine Übersicht und prospektive Studie zur Therapieeffizienz. Obere Extremität. 2013;8:122-128.

164. Dubert T, Houimli S, Valenti P, Dinh A. Very distal finger amputations: replantation or reposition flap repair? H Hand Surg. 1997;22(B):353-358.

165. Elliott $\mathrm{H}$, Norris $\mathrm{M}$, Kowalski T. Microsurgical managment of complex fingertip injuries: comparison to conventional skin grafting. J Reconstruc Microsurg. 1988;4:89-97.

166. Möller JB, Pers M, Schmidt A. Finger-tio injuries: late results. Acta Chir Scand. 1961;122:177183.

167. Suzuki K, Nanjo B, Nishibori M. A follow-up study of finger-tip injuries. Nihon Seikeigeka Gakkai Zasshi. 1965;38(13):1127-1133.

168. Cooper AF, Hankins M, Rixon L, Eaton E, Grunfeld EA. Distinct work-related, clinical and psychological factors predict return to work following treatment in four different cancer types. Psychooncology. 2013;22(3):659-667.

169. Parot-Schinkel E, Roquelaure $\mathrm{Y}, \mathrm{Ha} \mathrm{C}$, et al. Factors affecting return to work after carpal tunnel syndrome surgery in a large French cohort. Arch Phys Med Rehabil. 2011;92(11):1863-1869.

170. Seland K, Cherry N, Beach J. A study of factors influencing return to work after wrist or ankle fractures. Am J Ind Med. 2006;49(3):197-203.

171. Adani R, Busa R, Castagnetti C, Bathia A, Caroli A. Homodigital neurovascular island flaps with "direct flow" vascularization. Ann Plast Surg. 1997;38(1):36-40.

172. Nakanishi A, Omokawa S, Kawamura K, lida A, Kaji D, Tanaka Y. Tamai Zone 1 Fingertip Amputation: Reconstruction Using a Digital Artery Flap Compared With Microsurgical Replantation. The Journal of hand surgery. 2019;44(8):655-661. 
173. Braga-Silva J, Gehlen D, Bervian F, da Cunha GL, Padoin AV. Randomized prospective study comparing reverse and direct flow island flaps in digital pulp reconstruction of the fingers. Plast Reconstr Surg. 2009;124(6):2012-2018.

174. Sano K, Ozeki S, Kimura K, Hyakusoku H. Relationship between sensory recovery and advancement distance of oblique triangular flap for fingertip reconstruction. $J$ Hand Surg Am 2008;33:1088-1092.

175. Mermans JF, Franssen BB, Serroyen J, Van der Hulst RR. Digital nerve injuries: a review of predictors of sensory recovery after microsurgical digital nerve repair. Hand ( $N$ Y). 2012;7(3):233-241.

176. Han SK, Lee BI, Kim WK. The reverse digital artery island flap: clinical experience in 120 fingers. Plast Reconstr Surg. 1998;101(4):1006-1011; discussion 1012-1003.

177. Smith KL, Elliot D. The extended Segmuller flap. Plast Reconstr Surg. 2000;105(4):13341346.

178. Soderberg T, Nystrom A, Hallmans G, Hulten J. Treatment of fingertip amputations with bone exposure. A comparative study between surgical and conservative treatment methods. Scand J Plast Reconstr Surg. 1983;17(2):147-152.

179. Arbel R, Goodwin DR, Otremski I. Treatment of finger-tip injuries with silver sulphadiazine occlusion dressing. Injury. 1989;20(3):161-163.

180. Weichman KE, Wilson SC, Samra F, Reavey P, Sharma S, Haddock NT. Treatment and outcomes of fingertip injuries at a large metropolitan public hospital. Plast Reconstr Surg. 2013;131(1):107-112.

181. van den Berg WB, Vergeer RA, van der Sluis CK, Ten Duis HJ, Werker PM. Comparison of three types of treatment modalities on the outcome of fingertip injuries. $J$ Trauma Acute Care Surg. 2012;72(6):1681-1687. 


\section{Anhang}

\subsection{Ethikvotum}

\section{Ethik-Kommission bei der Landesärztekammer Hessen}

Landestratekammer Hessen, Im Vogelsgesang 3, 60488 Frankfurt/Main PeRsónLICH / VeRTRAULICH

Berufsgenossenschaftliche Unfallklinik Frankfurt am Main

Herrn Prof. Dr. med. Dr. med. habil. M. Sauerbier

Im Vogelsgesang 360488 Frankfurt am Main Postfach $900669 \quad 60486$ Frankfurt am Main Friedberger Landstr. 430

Telefon: 069 / $97672-209$ / 317 60389 Frankfurt/Main

$069 / 97672$ - 377

E-Mail: ethikkommission@laekh.de

Internet: www.laekh.de

Vorab per Telefax: 069 / 4754826

\begin{tabular}{|c|c|c|}
\hline Thr Zeichen & $\begin{array}{l}\text { (bitte Immer angeben) } \\
\text { Unser Zeichen: } \\
\text { III/1/sja/sla/ker }\end{array}$ & $\begin{array}{l}\text { Datum: } \\
\text { 7. Dezember } 2017\end{array}$ \\
\hline
\end{tabular}

FF 61/2016

Langzeitergebnisse nach plastisch-chirurgischer Defektdeckung durch neurovaskulären Insel-Lappen bei Fingerkuppenverletzungen

\section{Nachgereichte Unterlagen}

Thr Schreiben vom 01.11.2017, hier eingegangen am 06.11.2017

Sehr geehrter Herr Professor Sauerbier,

wir bestätigen den Eingang Ihres Schreibens vom 01.11.2017. Damit sind die Forderungen der Ethik-Kommission aus dem Schreiben vorn 13.07.2016 umgesetzt worden.

Gegen die Durchführung der Studie

Langzeitergebnisse nach plastisch-chirurgischer Defektdeckung durch neurovaskulären Insel-Lappen bei Fingerkuppenverletzungen

bestehen nunmehr weder berufsethischen noch berufsrechtlichen Bedenken.

Die Ethik-Kommission geht davon aus, dass nur einwilligungsfähige Patienten in die Studie eingeschlossen werden.

Die Ethik-Kommission bittet um zeitnahe Unterrichtung über alle schwerwiegenden oder unerwarteten unerwünschten Ereignisse, die während der Studie auftreten und die Sicherheit der Studienteilnehmer oder die Durchführung der Studie beeinträchtigen könnten. Dies gilt auch, wenn die. Studie aus unvorhergesehenen Gründen abgebrochen wird. 
Es wird darauf hingewiesen, dass Anderungen oder Erweiterungen des Versuchsplanes der Ethik-Kommission anzuzeigen sind und ggf. eine erneute Beratung erforderlich wird. Wir bitten, die einzureichenden Änderungen und/oder Erweiterungen der Studienunterlagen deutlich zu kennzeichnen.

Die Ethik-Kommission bittet außerdem nach Abschluss des Forschungsvorhabens um einen Bericht mit der Mitteilung der bei der Studie gewonnenen Ergebnisse.

Die ärzliche und juristische Verantwortung des Studienleiters und der an der Prüfung teilnehmenden Ärzte bleibt entsprechend der Beratungsfunktion der Ethik-Kommission durch unsere Stellungnahme unberührt.

An der Sitzung haben die in der Anlage aufgeführten Mitglieder der Ethik-Kommission teilgenommen. Der Beratung lagen die in der Anlage aufgeführten Unterlagen zugrunde.

Mit freundlichen Grüßen

i.A.

Prof. Dr. med. S. Harder

Vorsitzender der Ethik-Kommission 


\title{
12.2 Patienteninformation und Einwilligungserklärung
}

\section{Patienteninformation und Einwilligungserklärung zur klinischen Nachuntersuchung an volljährigen einwilligungsfähigen Patienten}

\author{
Studienzentrum: Berufsgenossenschaftliche Unfallklinik \\ Abteilung für Plastische, Hand- und Rekonstruktive Chirurgie \\ Friedberger Landstr. 430, 60389 Frankfurt am Main \\ Tel.: 0694752323 \\ Studienverantwortlicher: \\ Untersucher: \\ Prof. Dr. med. Dr. med. habil. Michael Sauerbier \\ Dr. med. Annika Arsalan-Werner (Prüfarzt) \\ Frau Natallia Brui (Doktorandin)
}

\section{Langzeitergebnisse nach plastisch-chirurgischer Defektdeckung durch neurovaskulären Insel-Lappen bei Fingerkuppenverletzungen}

Sehr geehrte Patientin, sehr geehrter Patient,

wir möchten Sie fragen, ob Sie bereit sind, an der nachfolgend beschriebenen wissenschaftlichen Studie teilzunehmen.

Wissenschaftliche Studien sind notwendig, um Erkenntnisse über die Sicherheit, Eignung und Leistungsfähigkeit von Operationsverfahren zu gewinnen oder zu erweitern.

Die Nachuntersuchung, die wir Ihnen hier vorstellen, wurde von einer Ethikkommission zustimmend bewertet. Diese Nachuntersuchung wird an der Berufsgenossenschaftlichen Unfallklinik Frankfurt am Main durchgeführt. Es sollen insgesamt ungefähr 50 Personen daran teilnehmen.

Ihre Teilnahme an dieser Studie ist freiwillig. Sie werden in diese Studie also nur dann einbezogen, wenn Sie dazu schriftlich Ihre Einwilligung erklären. Sofern Sie nicht an der Studie teilnehmen oder später aus ihr ausscheiden möchten, erwachsen Ihnen daraus keine Nachteile für Ihre weitere medizinische Behandlung.

Wenn Sie an der Studie teilnehmen, werden wir Sie klinisch nachuntersuchen und Sie bitten einen Fragebogen auszufüllen. Ihre bisher durchgeführten Röntgenuntersuchungen werden wir ebenfalls auswerten. Sie werden jedoch keiner zusätzlichen Röntgenbelastung ausgesetzt.

Wenn Sie die Teilnahme an der Studie ablehnen, erfolgt keine klinische Untersuchung. Dies hat keine Nachteile für Sie.

Sie wurden bereits auf die geplante Studie angesprochen. Der nachfolgende Text soll Ihnen die Ziele und den Ablauf erläutern. Anschließend wird ein Prüfarzt oder ein(e) von inm benannte(r) Untersucher(in) das Aufklärungsgespräch mit Ihnen führen. Bitte zögern Sie nicht, alle Punkte anzusprechen, die Ihnen unklar sind. Sie werden danach ausreichend Bedenkzeit erhalten, um über Ihre Teilnahme zu entscheiden. 


\section{Warum wird diese Nachuntersuchung durchgeführt?}

In dieser Studie zu Defektdeckungen von Fingerkuppenverletzungen mit dem neurovaskulären Insel-Lappen sollen die Langzeitergebnisse der Operationsmethode hinsichtlich der funktionellen Resultate und die subjektive Zufriedenheit der Patienten nach erfolgter Versorgung untersucht werden.

Da es zu diesem Thema bisher nur wenige Langzeitergebnisse gibt und an dieser Klinik besonders viele Patienten mit diesen Verletzungen versorgt wurden, ist es von großem Interesse, die Langzeitergebnisse hinsichtlich der funktionellen Ergebnisse und Patientenzufriedenheit nach plastisch-chirurgischer Defektdeckung mit dem neurovaskulären Insel-Lappen zu beurteilen.

Zusätzlich werden die schon vorhandenen Röntgenbilder in Hinblick auf die Verletzungshöhe untersucht.

\section{Wie ist der Ablauf der Studie und was muss ich bei Teilnahme beachten?}

Bei Aufnahme in diese Studie wird die Vorgeschichte Ihrer Krankheit erhoben sowie die vorhandene Patientenakte ausgewertet. Die schon vorhandenen Röntgenbilder werden ebenfalls untersucht.

Sie werden einer umfassenden ärztlichen Untersuchung unterzogen mit speziellem Fokus auf den operierten Finger. Zusätzlich erhalten Sie einen speziellen Fragenbogen bezüglich möglicher Beschwerden bzw. Ihrer Fähigkeit, bestimmte Tätigkeiten auszuführen (DASH-Fragebogen). Das Ausfüllen dieses Fragebogens nimmt etwa 10 Minuten in Anspruch.

Eine zusätzliche Röntgenuntersuchung ist nicht notwendig, daher fällt keine zusätzliche Strahlenbelastung an.

Bei Teilnahme an der Studie erfolgt für Sie ein Untersuchungstermin. Die Untersuchung wird etwa 45 Minuten dauern.

\section{Welche Risiken sind mit der Teilnahme an der Studie verbunden?}

Die Teilnahme an der Studie birgt keine speziellen Risiken.

\section{Entstehen für mich Kosten durch die Teilnahme an der Studie? Erhalte ich eine Aufwandsentschädigung?}

Durch Ihre Teilnahme an dieser Studie entstehen für Sie Reisekosten, welche nicht übernommen werden können. Eine Aufwandsentschädigung wird nicht gezahlt.

\section{Bin ich während der Studie versichert?}

Versicherungsschutz besteht über eine Wegeunfall-Versicherung. Die Versicherung umfasst ausschließlich Wegeunfälle, von denen die versicherten Personen auf dem Weg zum und vom Prüfungsort für ihre Teilnahme an der Prüfung betroffen werden. Der Versicherungsschutz beginnt jeweils, sobald der Versicherte zum Zwecke des Antritts der Reise seine Wohnung verlässt, jedoch nicht früher als 24 Stunden vor Beginn an der Teilnahme der Prüfung. Der Versicherungsschutz endet mit Rückkehr in die Wohnung, spätestens jedoch 24 Stunden nach Beendigung der Teilnahme an der Prüfung. 
Ansprechpartner im Versicherungsfall ist unsere hauseigene Stabsstelle Vertragsmanagement.

\section{Was geschieht mit meinen Daten?}

Ihre personenbezogenen Daten werden insgesamt spätestens 4 Jahre nach ihrer erstmaligen Erhebung gelöscht - gegebenenfalls auch früher, sofern die Auswertung dann schon erfolgt sein sollte. Ihre Daten werden während der Studie in Form medizinischer Befunde und persönlicher Informationen erhoben und in der Prüfstelle in Ihrer persönlichen Akte niedergeschrieben oder elektronisch gespeichert. Die für die Studie wichtigen Daten werden zusätzlich in pseudonymisierter Form - also ohne persönliche Angaben wie Name oder Initialen - gespeichert und an das Institut für Biostatistik der Johann-Wolfgang-Goethe-Universität Frankfurt am Main zwecks weiterer Auswertung weitergegeben. Die Daten sind gegen unbefugten Zugriff gesichert. Der Kreis der zugriffsberechtigten Personen umfasst den Studienleiter (Prof. Dr. Dr. M. Sauerbier), die Prüfärztin (Dr. med. A. Arsalan-Werner) und die Untersucherin (Frau N. Brui).

Das Pseudonymisierungsverfahren sieht vor, dass die gesammelten Informationen unter Obhut der zugriffsberechtigten Personen in eine Tabelle mit reduziertem Informationsgehalt übertragen werden, in der eine neu erstellte Pseudonymisierungsnummer sämtliche personenbezogenen Daten (Name, Geburtsdatum, Aufnahmenummer u.s.w.) ersetzt. Erst diese pseudonymisierte Liste wird zur Auswertung weitergegeben. Ein Rückschluss auf personenbezogene Daten ist daraus nicht möglich.

Bei Widerruf in die Einwilligung zur Studienteilnahme werden Ihre personenbezogenen Daten gelöscht. Sofern der Widerruf nach der statistischwissenschaftlichen Auswertung erfolgt ist eine Löschung von anderen als den personenbezogenen Daten nicht möglich.

Gemäß Bundesdatenschutzgesetz steht Ihnen ein Recht auf Einsicht und - im Falle unrichtiger Dokumentation - auf Korrektur ihrer Daten zu.

\section{An wen wende ich mich bei weiteren Fragen?}

Dr. med. Annika Arslan-Werner

Tel.: 069/475-2021

Frau Natallia Brui

Tel.: 069/475-2021

Prof. Dr. med. Dr. med. habil. Michael Sauerbier

Tel.: 069/475-2021

Abteilung für Plastische, Hand- und Rekonstruktive Chirurgie Berufsgenossenschaftliche Unfallklinik

Friedberger Landstr. 430

60389 Frankfurt am Main 


\title{
Langzeitergebnisse nach plastisch-chirurgischer Defektdeckung von Fingerkuppen- Verletzungen mit dem neurovaskulären Insel-Lappen
}

\author{
- Einwilligungserklärung - \\ Patient/in: \\ geboren am: \\ Name des aufklärenden Arztes:
}

Ich, der/die o.g. Patient/in, wurde in einem ausführlichen Gespräch durch den oben genannten Arzt über Inhalt, Bedeutung, Durchführung, mögliche Vorteile und Risiken der Untersuchung nach Versorgung Fingerkuppendefekten und plastischchirurgischer Defektdeckung mit neurovaskulärem Insel-Lappen aufgeklärt.

Ich hatte ausreichend Zeit Fragen zu stellen und meine Entscheidung zu überdenken. Alle offenen Fragen konnten mit dem behandelnden Arzt besprochen werden.

Ich werde den ärztlichen Anweisungen, die für die Durchführung der Studie erforderlich sind, Folge leisten, behalte mir jedoch das Recht vor, meine freiwillige Mitwirkung jederzeit zu beenden, ohne dass mir dadurch Nachteile in meiner medizinischen Versorgung entstehen.

Ich bin damit einverstanden, dass ich zu den Nachuntersuchungen schriftlich oder fernmündlich eingeladen werde, ohne dass sich hieraus Verpflichtungen meinerseits ergeben.

Ein Exemplar der Patienteninformation und Einwilligungserklärung wurde mir ausgehändigt. Das Original verbleibt in der Klinik.

\section{Datenschutz:}

Ich bin mit der Aufzeichnung der im Rahmen der Studie an mir erhobenen Krankheitsdaten und ihrer pseudonymisierten Verwendung für wissenschaftliche Zwecke einverstanden. Pseudonymisiert bedeutet, dass keine Angaben von Namen oder Initialen verwendet werden, sondern nur ein Nummern- und/oder Buchstabencode evtl. mit Angabe des Geburtsjahres.

Meine Daten dürfen pseudonymisiert und unter Verschluss in der BGU Frankfurt aufbewahrt werden. Mein Name wird zu keiner Zeit öffentlich gemacht. Die Schlüsseltabelle zur Rückführung der Kennnummern auf die Namen wird unter Verschluss beim Leiter des Projektes in der BGU oder einer von inm benannten Vertrauensperson gelagert. Somit ist in dringenden Fällen eine schnelle Rückführung auf den Namen möglich.

Alle Personen, die Einblick in die gespeicherten Daten haben, sind zur Wahrung des Datenschutzes verpflichtet. Insbesondere autorisierte Beauftragte des Prüfarztes sowie die zuständigen Überwachungsbehörden, soweit dies für die Überprüfung der ordnungsgemäßen Durchührung der Studie notwendig ist. Ich entbinde von der Schweigepflicht meiner Patientendaten und erlaube die retrospektive Auswertung der Daten durch die im nächsten Absatz benannten Personen.

Der Studienleiter ist Prof. Dr. med. Dr. med. habil. M. Sauerbier (BGU - Frankfurt). Die von inm benannten Vertrauenspersonen sind die Prüfärztin Dr. med. Annika Arsalan-Werner (BGU - Frankfurt) und als weitere Untersucherin Frau N. Brui (BGU - Frankfurt). 
Eine Löschung der Daten wird spätestens 4 Jahre nach ihrer Erhebung, soweit nicht gesetzmäßige oder satzungsmäßige Aufbewahrungsfristen entgegenstehen, erfolgen. Ich bin darüber informiert worden, dass ich jederzeit das Recht habe, meine Einwilligung zur Studienteilnahme ohne Angabe von Gründen zu widerrufen, ohne dass mir hieraus Nachteile für meine weitere medizinische Behandlung erwachsen. Meine bis dahin erhobenen Daten werden gelöscht.

Gemäß Bundesdatenschutzgesetz habe ich das Recht auf Auskunft über meine durch die BGU-Frankfurt gespeicherten Daten sowie das Recht auf Berichtigung unrichtiger Daten bzw. auf Löschung/Sperrung unzulässig gespeicherter Daten.

Ich erkläre mich freiwillig bereit, an der o.g. Studie teilzunehmen.

Ort, Datum und Uhrzeit:

Unterschrift des/r Patienten/in:

Ort, Datum und Uhrzeit:

Unterschrift des Arztes: 


\subsection{DASH Fragebogen}

\section{Disabilities of the Arm, Shoulder and Hand}

\section{THE}

\section{ANLEITUNC}

Dieser Fragebogen beschäftigt sich sowohl mit Ihren Beschwerden als auch mit Ihren Fähigkeiten, bestimmte Tätigkeiten auszuführen.

Bitte beantworten Sie alle Fragen gemäß Ihrem Zustand in der vergangenen Woche, indem Sie einfach die entsprechende Zahl ankreuzen.

Wenn Sie in der vergangenen Woche keine Gelegenheit gehabt haben, eine der unten aufgeführten Tätigkeiten durchzuführen, so wählen Sie die Antwort aus, die Ihrer Meinung nach am ehesten zutreffen würde.

Es ist nicht entscheidend, mit welchem Arm oder welcher Hand Sie diese Tätigkeiten ausüben. Antworten Sie Ihrer Fähigkeit entsprechend, ungeachtet, wie Sie die Aufgaben durchführen konnten. 


\section{Disabilities of the Arm, Shoulder and Hand}

Bitte schätzen Sie Ihre Fähigkeit ein, wie Sie folgende Tätigkeiten in der vergangenen Woche durchgeführt haben, indem Sie die entsprechende Zahl ankreuzen.

\begin{tabular}{|c|c|c|c|c|c|}
\hline & \\
\hline & $\begin{array}{l}\text { Keine } \\
\text { chwierig- } \\
\text { keiten }\end{array}$ & $\begin{array}{l}\text { Geringe } \\
\text { Schwierig- } \\
\text { keiten }\end{array}$ & $\begin{array}{l}\text { Mäßige } \\
\text { Schwierig- } \\
\text { keiten }\end{array}$ & $\begin{array}{l}\text { Erhebliche } \\
\text { Schwierig- } \\
\text { keiten }\end{array}$ & $\begin{array}{c}\text { Nicht } \\
\text { möglich }\end{array}$ \\
\hline $\begin{array}{l}\text { 1. Ein neues oder } \\
\text { festverschlossenes Glas öffnen }\end{array}$ & 1 & 2 & 3 & 4 & 5 \\
\hline 2. Schreiben & 1 & 2 & 3 & 4 & 5 \\
\hline 3. Einen Schlüssel umdrehen & 1 & 2 & 3 & 4 & 5 \\
\hline 4. Eine Mahlzeit zubereiten & 1 & 2 & 3 & 4 & 5 \\
\hline 5. Eine schwere Tür aufstoßen & 1 & 2 & 3 & 4 & 5 \\
\hline $\begin{array}{l}\text { 6. Einen Gegenstand über } \\
\text { Kopfhöhe auf ein Regal stellen }\end{array}$ & 1 & 2 & 3 & 4 & 5 \\
\hline $\begin{array}{l}\text { 7. Schwere Hausarbeit (z. B Wände } \\
\text { abwaschen, Boden putzen) }\end{array}$ & 1 & 2 & 3 & 4 & 5 \\
\hline 8. Garten- oder Hofarbeit & 1 & 2 & 3 & 4 & 5 \\
\hline 9. Betten machen & 1 & 2 & 3 & 4 & 5 \\
\hline $\begin{array}{l}\text { 10. Eine Einkaufstasche oder } \\
\text { einen Aktenkoffer tragen }\end{array}$ & 1 & 2 & 3 & 4 & 5 \\
\hline $\begin{array}{l}\text { 11. Einen schweren Gegenstand } \\
\text { tragen (über } 5 \mathrm{~kg} \text { ) }\end{array}$ & 1 & 2 & 3 & 4 & 5 \\
\hline $\begin{array}{l}\text { 12. Eine Glühbirne über Ihrem } \\
\text { Kopf auswechseln }\end{array}$ & 1 & 2 & 3 & 4 & 5 \\
\hline $\begin{array}{l}\text { 13. Ihre Haare waschen } \\
\text { oder fönen }\end{array}$ & 1 & 2 & 3 & 4 & 5 \\
\hline 14. Ihren Rücken waschen & 1 & 2 & 3 & 4 & 5 \\
\hline 15. Einen Pullover anziehen & 1 & 2 & 3 & 4 & 5 \\
\hline $\begin{array}{l}\text { 16. Ein Messer benutzen, um } \\
\text { Lebensmittel zu schneiden }\end{array}$ & 1 & 2 & 3 & 4 & 5 \\
\hline $\begin{array}{l}\text { 17. Freizeitaktivitäten, die wenig } \\
\text { körperliche Anstrengung } \\
\text { verlangen (z. B. Karten spielen, } \\
\text { Stricken, usw.) }\end{array}$ & 1 & 2 & 3 & 4 & 5 \\
\hline $\begin{array}{l}\text { 18. Freizeitaktivitäten, bei denen auf } \\
\text { Ihren Arm, Schulter oder Hand } \\
\text { Druck oder Stoß ausgeübt wird } \\
\text { (z.B. Golf, Hämmern, Tennis, usw.) }\end{array}$ & 1 & 2 & 3 & 4 & 5 \\
\hline $\begin{array}{l}\text { 19. Freizeitaktivitäten, bei denen } \\
\text { Sie Ihren Arm frei bewegen } \\
\text { (z. B. Badminton, Frisbee) }\end{array}$ & 1 & 2 & 3 & 4 & 5 \\
\hline $\begin{array}{l}\text { 20. Mit Fortbewegungsmitteln } \\
\text { zurecht zukommen (um } \\
\text { von einem Platz zum } \\
\text { anderen zu gelangen) }\end{array}$ & 1 & 2 & 3 & 4 & 5 \\
\hline 21. Sexuelle Aktivität & 1 & 2 & 3 & 4 & 5 \\
\hline
\end{tabular}




\section{Disabilities of the ARM, Shoulder and Hand}

22. In welchem Ausmaß haben Ihre Schulter-, Arm- oder Handprobleme Ihre normalen sozialen

Aktivitäten mit Familie, Freunden, Nachbarn oder anderen Gruppen während der

vergangenen Woche beeinträchtigt? (Bitte kreuzen Sie die entsprechende Zahl an)

\begin{tabular}{ccccc}
\hline $\begin{array}{c}\text { Überhaupt } \\
\text { nicht }\end{array}$ & Ein wenig & Mäßig & Ziemlich & Sehr \\
1 & 2 & 3 & 4 & 5 \\
\hline
\end{tabular}

23. Waren Sie in der vergangenen Woche durch Ihre Schulter-, Arm- oder Handprobleme in Ihrer Arbeit oder anderen alltäglichen Aktivitäten eingeschränkt? (Bitte kreuzen Sie die entsprechende Zahl an)

\begin{tabular}{ccccc}
\hline $\begin{array}{c}\text { Überhaupt } \\
\text { nicht } \\
\text { eingeschränkt }\end{array}$ & $\begin{array}{c}\text { Ein } \\
\text { wenig } \\
\text { eingeschränkt }\end{array}$ & $\begin{array}{c}\text { Mäßig } \\
\text { eingeschränkt }\end{array}$ & $\begin{array}{c}\text { Sehr } \\
\text { eingeschränkt }\end{array}$ & $\begin{array}{c}\text { Nicht } \\
\text { möglich }\end{array}$ \\
1 & 2 & 3 & 4 & 5
\end{tabular}

Bitte schätzen Sie die Schwere der folgenden Beschwerden während

der letzten Woche ein. (Bitte kreuzen Sie in jeder Zeile die entsprechende Zahl an)

\begin{tabular}{lccccc} 
& Keine & Leichte & Mäßige & Starke & Sehr starke \\
\hline $\begin{array}{l}\text { 24. Schmerzen in Schulter, Arm } \\
\text { oder Hand }\end{array}$ & 1 & 2 & 3 & 4 & 5 \\
$\begin{array}{l}\text { 25. Schmerzen in Schulter, Arm } \\
\text { oder Hand während der }\end{array}$ & & & & \\
$\begin{array}{l}\text { Ausführung einer } \\
\text { bestimmten Tätigkeit }\end{array}$ & $\mathbf{1}$ & 2 & $\mathbf{3}$ & 4 & 5
\end{tabular}

26. Kribbeln (Nadelstiche)

in Schulter, Arm

oder Hand

(1)
27. Schwächegefühl in Schulter,
Arm oder Hand

1
oder Hand

1

2

3

4

5

2

3

$4 \quad 5$

29. Wie groß waren Ihre Schlafstörungen in der letzten Woche aufgrund von Schmerzen im Schulter-, Arm- oder Handbereich? (Bitte kreuzen Sie die entsprechende Zahl an)

\begin{tabular}{ccccc}
\hline $\begin{array}{c}\text { Keine } \\
\text { Schwierig- } \\
\text { keiten }\end{array}$ & $\begin{array}{c}\text { Geringe } \\
\text { Schwierig- } \\
\text { keiten }\end{array}$ & $\begin{array}{c}\text { Mäßige } \\
\text { Schwierig- } \\
\text { keiten }\end{array}$ & $\begin{array}{c}\text { Erhebliche } \\
\text { Schwierig- } \\
\text { keiten }\end{array}$ & $\begin{array}{c}\text { Nicht } \\
\text { möglich }\end{array}$ \\
1 & 2 & 3 & 4 & 5 \\
\hline
\end{tabular}

30. Aufgrund meiner Probleme im Schulter-, Arm- oder Handbereich empfinde ich meine Fähigkeiten als eingeschränkt, ich habe weniger Selbstvertrauen oder ich fühle, dass ich mich weniger nützlich machen kann. (Bitte kreuzen Sie die entsprechende Zahl an)

\begin{tabular}{ccccc}
\hline $\begin{array}{c}\text { Stimme } \\
\text { uberhaupt } \\
\text { nicht zu }\end{array}$ & $\begin{array}{c}\text { Stimme } \\
\text { nicht } \\
\text { zu }\end{array}$ & $\begin{array}{c}\text { Weder Zustimmung } \\
\text { noch Ablehnung }\end{array}$ & $\begin{array}{c}\text { Stimme } \\
\text { zu }\end{array}$ & $\begin{array}{c}\text { Stimme } \\
\text { sehr zu }\end{array}$ \\
1 & 2 & 3 & 4 & 5 \\
\hline
\end{tabular}




\section{Disabilities of the Arm, Shoulder and Hand}

\section{ARBEITS- UND BERUFS-MODUL (OPTIONAL)}

Die folgenden Fragen beziehen sich auf den Einfluss Ihres Schulter-, Arm- oder Handproblems auf Ihre Arbeit_ (einschließlich Haushaltsführung, falls dies Ihre Hauptbeschäftigung ist).

Bitte geben Sie Ihre/n Arbeit/Beruf hier an:

$\square$ Ich bin nicht berufstätig (Sie können diesen Bereich auslassen).

Bitte kreuzen Sie die Zahl an, die Ihre körperlichen Fähigkeiten in der vergangenen Woche am besten beschreibt. Hatten Sie irgendwelche Schwierigkeiten:

\begin{tabular}{|c|c|c|c|c|c|c|}
\hline & \multirow[b]{2}{*}{$\begin{array}{l}\text { Keine } \\
\text { Schwierig- } \\
\text { keiten }\end{array}$} & & \multirow[b]{2}{*}{$\begin{array}{l}\text { Erhebliche } \\
\text { Schwierig- } \\
\text { keiten }\end{array}$} & \multirow[b]{2}{*}{$\begin{array}{l}\text { Nicht } \\
\text { möglich }\end{array}$} \\
\hline & & & $\begin{array}{l}\text { Geringe } \\
\text { Schwierig- } \\
\text { keiten }\end{array}$ & $\begin{array}{l}\text { Mäßige } \\
\text { Schwierig- } \\
\text { keiten }\end{array}$ & & \\
\hline 1. & $\begin{array}{l}\text { In der üblichen Art und } \\
\text { Weise zu arbeiten? }\end{array}$ & 1 & 2 & 3 & 4 & 5 \\
\hline 2. & $\begin{array}{l}\text { Aufgrund der Schmerzen in } \\
\text { Schulter, Arm oder Hand Ihre } \\
\text { übliche Arbeit zu erledigen? }\end{array}$ & 1 & 2 & 3 & 4 & 5 \\
\hline 3. & $\begin{array}{l}\text { So gut zu arbeiten } \\
\text { wie Sie es möchten? }\end{array}$ & 1 & 2 & 3 & 4 & 5 \\
\hline & $\begin{array}{l}\text { Die bisher gewohnte Zeit mit } \\
\text { Ihrer Arbeit zu verbringen? }\end{array}$ & 1 & 2 & 3 & 4 & 5 \\
\hline
\end{tabular}

\section{SPORT- UND MUSIK-MODUL (OPTIONAL)}

Die folgenden Fragen beziehen sich auf den Einfluss Ihres Schulter-, Arm- oder Handproblems auf das Spielen Ihres Musikinstrumentes oder auf das Ausüben Ihres Sports oder auf beides.

Wenn Sie mehr als ein Instrument spielen oder mehr als eine Sportart ausüben (oder beides), so beantworten Sie bitte die Fragen in bezug auf das Instrument oder die Sportart, die für Sie am wichtigsten ist.

Bitte geben Sie dieses Instrument bzw. diese Sportart hier an:

$\square$ Ich treibe keinen Sport oder spiele kein Instrument (Sie können diesen Bereich auslassen)

Bitte kreuzen Sie die Zahl an, die Ihre körperlichen Fähigkeiten in der vergangenen Woche am besten beschreibt. Hatten Sie irgendwelche Schwierigkeiten:

\begin{tabular}{ccccc}
\hline Keine & Geringe & Mäßige & Erhebliche & Nicht \\
$\begin{array}{c}\text { Schwierig- } \\
\text { keiten }\end{array}$ & $\begin{array}{c}\text { Schwierig- } \\
\text { keiten }\end{array}$ & $\begin{array}{c}\text { Schwierig- } \\
\text { keiten }\end{array}$ & $\begin{array}{c}\text { Schwierig- } \\
\text { keiten }\end{array}$ & möglich \\
\hline
\end{tabular}

1. In der üblichen Art und Weise Ihr Musikinstrument zu spielen oder Sport zu treiben?

1

2

3

4

2. Aufgrund der Schmerzen in Schulter, Arm oder Hand Ihr Musikinstrument zu spielen oder Sport zu treiben?

1

2

4 5

3. So gut Ihr Musikinstrument zu spielen oder Sport zu treiben wie Sie es möchten?

1

3

4. Die bisher gewohnte Zeit mit dem Spielen Ihres Musikinstrumentes oder mit Sporttreiben zu verbringen?

2 3 4
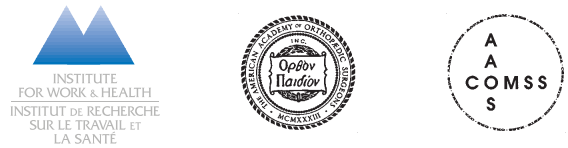

19

DASH Deutsche Version 2002:
Guenter Germann, Angela Hart Gerhard Wind, Erhan Demir Klinik für Hand-, Plastische
Rekonstruktive Chirugrie

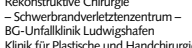

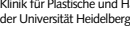




\subsection{Patientenuntersuchungsbogen}

\section{Patientenuntersuchungsbogen}

Welcher Finger ist verletzt?

Rechts: DI_DII_DIII_DIV_DV, Links: DI_DII_DIII_DIV_DV

Art der Verletzung:

Händigkeit:

DASH-Bogen ausgefüllt:

Beruf vor Verletzung:

Beruf nach Verletzung:

Gewechselt wegen Verletzung? Ja__ Nein

Arbeitsunfall: Ja/nein

Zeit in Wochen von Unfall bis zur endgültigen Heilung: Wochen

Zeit in Wochen von Unfall bis zur Arbeitsfähigkeit: Wochen

Anzahl Physiotherapie insgesamt: ___ Mal (_ h)

Anzahl Ergotherapie insgesamt: ___ Mal (_

Zum Zeitpunkt der Lappenplastik geraucht: ja/nein, wie viel

Lag der Knochen frei? Ja/nein

Nachbehandlung? Welcher Verband?

Komplikationen? Ja/nein

Operationen bei uns nach der initialen OP? Ja/nein

Operationen auswärts nach der initialen OP? Ja/nein 
Im Verlauf Infektion, wie therapiert? Ja/nein

Nicht operativ therapierte Komplikationen? Ja/nein

Probleme an der Entnahmestelle, z.B. Schmerzen, Missempfindung,

subjektives Hoffmann-Tinel-Zeichen?

Zufriedenheit: $\ldots(0=$ maximal unzufrieden, $10=$ maximal zufrieden $)$

Schmerzen in Ruhe: ___ ( $0=$ keine, $10=$ maximal vorstellbare Schmerzen $)$

Schmerzen unter Belastung: ___ $(0=$ keine, $10=$ maximal vorstellbare

Schmerzen)

Würden Sie sich wieder operieren lassen? Ja/nein

Zufrieden mit der Operation? Ja/nein

Verletzung vor dieser Verletzung/Vorerkrankungen:

Version 1.2

Anhang
Seite 2 von 6

- 123 - 


\section{Untersuchung}

\section{Foto-Dokumentation:}

Verletzter Finger mit Gegenseite von dorsal, seitlich und palmar.

Klassifikation nach Allen:

$\underline{\text { Inspektion/Palpation verletzter Finger: }}$

Narbe: schmerzhaft, gerötet

Vancover Scar Scale

\begin{tabular}{|l|l|l|l|}
\hline Pigmentierung & Durchblutung & Geschmeidigkeit & Höhe \\
\hline 0 normal & 0 normal & 0 normal & 0 normal \\
\hline 2 Hypopigmentierung & 1 rosa & 1 geschmeidig & $1<2 \mathrm{~mm}$ \\
\hline 3 Hyperpigmentierung & 2 rot & $\begin{array}{l}2 \text { nachgebend bei } \\
\text { Druck }\end{array}$ & $2<5 \mathrm{~mm}$ \\
\hline & 3 lila/violett & 3 steif & $3>5 \mathrm{~mm}$ \\
\hline & & 4 hart & \\
\hline & & 5 kontrakt & \\
\hline
\end{tabular}

Gesamtzahl:

Schwellung: keine - leicht - mäßig - stark

Druckschmerz? Ja/nein

Kälteempfindlichkeit? Ja/nein

Hoffmann-Tinel-Zeichen? Ja/nein

Version 1.2

Anhang
Seite 3 von 6

$-124-$ 
Schweissbildung:

Subjektiv normal___erhöht___vermindert

Objektiv normal__erhöht___vermindert

\section{Temperatur:}

Subjektiv normal__überwärmt___ühl

Objektiv normal__überwärmt__ kühl

Lappengröße:

$\ldots$ ___

Nagelwachstumsstörung:

Wenn ja: Beschreibung mit Entzündung? Wachstum? Hängenbleiben?

Beschwerden? Rillen? Furchen? Krallennagel?

Länge des Nagels

\begin{tabular}{|c|c|c|}
\hline & rechts & links \\
\hline \multirow[t]{2}{*}{ DI } & Ja/nein & Ja/nein \\
\hline & Länge im mm: & Länge im mm: \\
\hline \multirow[t]{2}{*}{ DII } & Ja/nein & Ja/nein \\
\hline & Länge im mm: & Länge im mm: \\
\hline \multirow[t]{2}{*}{ DIII } & Ja/nein & Ja/nein \\
\hline & Länge im mm: & Länge im mm: \\
\hline \multirow[t]{2}{*}{ DIV } & Ja/nein & Ja/nein \\
\hline & Länge im mm: & Länge im mm: \\
\hline \multirow[t]{2}{*}{ DV } & Ja/nein & Ja/nein \\
\hline & Länge im mm: & Länge im mm: \\
\hline
\end{tabular}

Version 1.2

Seite 4 von 6

Anhang

-125 - 
Semmes-Weinstein-Test

\begin{tabular}{|l|l|l|l|l|}
\hline & Rechts radial & Rechts ulnar & Links radial & Links ulnar \\
\hline DI & & & & \\
\hline DII & & & & \\
\hline DIII & & & & \\
\hline DIV & & & & \\
\hline DV & & & & \\
\hline
\end{tabular}

2-Punkt-Diskrimination:

\begin{tabular}{|l|l|l|l|l|}
\hline & Rechts radial & Rechts ulnar & Links radial & Links ulnar \\
\hline DI & & & & \\
\hline DII & & & & \\
\hline DIII & & & & \\
\hline DIV & & & & \\
\hline DV & & & & \\
\hline
\end{tabular}

Handgelenk:

\begin{tabular}{|l|l|l|}
\hline & rechts & links \\
\hline HG Dorsalextension & & \\
\hline HG Palmarflexion & & \\
\hline HG Radialabduktion & & \\
\hline HG Ulnarabduktion & & \\
\hline HG Supination & & \\
\hline HG Pronation & & \\
\hline
\end{tabular}

Fingerbeweglichkeit:

\begin{tabular}{|l|l|l|}
\hline & rechts & links \\
\hline DII MCP & & \\
\hline DII PIP & & \\
\hline DII DIP & & \\
\hline DIII GG & & \\
\hline DIII PIP & & \\
\hline DIII DIP & & \\
\hline
\end{tabular}

Version 1.2

Seite 5 von 6

Anhang

$-126-$ 


\begin{tabular}{|l|l|l|}
\hline DIV MCP & & \\
\hline DIV PIP & & \\
\hline DIV DIP & & \\
\hline DV MCP & & \\
\hline DV PIP & & \\
\hline DV DIP & & \\
\hline
\end{tabular}

Daumenbeweglichkeit:

\begin{tabular}{|l|l|l|}
\hline & rechts & links \\
\hline Daumen IP & & \\
\hline Daumen MCP & Welche Fingerspitze wird & $\begin{array}{l}\text { Welche Fingerspitze wird } \\
\text { erreicht? } \\
\text { Sattelgelenk }\end{array}$ \\
& $\begin{array}{l}\text { II III IV V III IV V } \\
\text { Kapandji-Index: }\end{array}$ & Kapandji-Index: \\
\hline
\end{tabular}

\section{Kraftmessung:}

\begin{tabular}{|l|l|l|}
\hline & rechts & links \\
\hline $\begin{array}{l}\text { Jamar Dynamometer } \\
\text { Stufe II (kg) }\end{array}$ & 1. & 1. \\
\hline & 2. & 2. \\
\hline & 3. & 3. \\
\hline & Durchschnitt: & Durchschnitt: \\
\hline Pinch Gauge (kg) & & \\
\hline I-II & & \\
\hline I-III & & \\
\hline I-IV & & \\
\hline I-V & & \\
\hline
\end{tabular}

Anhang Version $1.2 \quad$ - \$2 $127-$ von 6




\title{
13 Publikation
}

Archives of Orthopaedic and Trauma Surgery https://doi.org/10.1007/s00402-019-03198-4

HANDSURGERY

\section{Long-term outcome of fingertip reconstruction with the homodigital neurovascular island flap}

\author{
Annika Arsalan-Werner ${ }^{1} \cdot$ Natallia Brui $^{1} \cdot$ Isabella Mehling $^{2} \cdot$ Michael Schlageter $^{1} \cdot$ Michael Sauerbier $^{1}$ \\ Received: 1 April 2019 \\ ○) Springer-Verlag GmbH Germany, part of Springer Nature 2019
}

\begin{abstract}
Introduction Fingertip injuries are frequent and several surgical strategies exist to reconstruct the amputated part and restore function and appearance. Yet, long-term results are rarely published. The purpose of this study was to examine the long-term clinical outcome of neurovascular island flaps for traumatic fingertip amputation of Allen type III/IV injuries.

Materials and methods We retrospectively analysed a cohort of patients with traumatic fingertip amputation that underwent reconstruction with a neurovascular island flap from January 2003 to December 2014. No mandatory splinting was applied after surgery. 28 participants (29 fingers) were available for follow-up at mean 8 years after reconstruction. Activities of daily living were measured with the disabilities of the arm, shoulder and hand questionnaire. Grip strength and finger motion were assessed using a Jamar dynamometer and a goniometer. Two-point discrimination and Semmes-Weinstein monofilaments were used to evaluate sensory recovery.

Results No intraoperative complications occurred and all flaps survived. Mean flap size was $4.7 \pm 0.6 \mathrm{~cm}^{2}$. Active motion of the fingers was over $95 \%$ of the contralateral side at follow-up. Three patients showed mild extension lag of the proximal interphalangeal joint. The grip strength of the affected hand and of each of the affected fingers was over $70 \%$ of the contralateral side. In comparison to the contralateral side we did not detect any significant difference for the Semmes-Weinstein monofilament test, but two-point discrimination $(5.1 \pm 1.7 \mathrm{~mm})$ was significantly impaired. According to the Lim classification 1 of 14 nails with hook nail deformity showed grade 3 breaking of the nail. The DASH score was 16.0. All patients returned to their original occupation and patient satisfaction with the procedure was high.

Conclusions The risk for disabling flexion contracture seems to be small even without mandatory splinting. Neurovascular island flaps for fingertip amputation of Allen type III/IV injuries are a reliable tool in fingertip reconstruction in the long term.
\end{abstract}

Keywords Fingertip defect · Homodigital neurovascular island flap · Homodigital artery flap · Fingertip reconstruction · Direct flow flap

\section{Introduction}

Fingertip amputations are frequent injuries in trauma units and require immediate treatment $[1,2]$. Several surgical strategies exist to reconstruct the amputated part and restore

\footnotetext{
$\triangle$ Annika Arsalan-Werner

annika.arsalan-werner@bgu-frankfurt.de

1 Department for Plastic, Hand and Reconstructive Surgery, Hand Trauma Center, BG-Trauma Center Frankfurt am

Main, Academic Hospital of the Johann Wolfgang GoetheUniversity Frankfurt Am Main, Friedberger Landstrasse 430, 60389 Frankfurt am Main, Germany

2 Division for Hand Surgery, St. Vinzenz Hospital Hanau, Am Frankfurter Tor 19, 63450 Hanau, Germany
}

function and appearance. The ideal reconstruction aims to replace "like with like" and thus to recreate a near normal fingertip in one operation with immediate sensate skin, minimal donor side morbidity and near normal movement [3].

The homodigital island flap meets most of these criteria as it is a sensate flap from the same finger with glabrous tissue to reconstruct the fingertip. Yet many studies report some extent of proximal interphalangeal (PIP) joint flexion contracture due to bending of the finger for an optimal insertion of the flap. Some authors advocate to include z-plasties [4] or step ladder designs [5] or to implement a mandatory splinting regime [6] to minimize PIP joint contracture. However, most studies reporting clinical outcomes of the homodigital neurovascular island flap for fingertip reconstruction only report short or mid-term follow-up and do no 
specify the type of donor site closure or the postoperative splinting regime $[4,7-10]$.

In this study, we evaluated the long-term clinical outcome of patients with Allen type III/IV finger amputation injuries [11], focussing on the recovery in range of motion, sensibility and impact on daily life.

\section{Methods}

We retrospectively analysed a cohort of 53 consecutive patients with traumatic fingertip amputation that underwent reconstruction with a neurovascular island flap from January 2003 to December 2014.

The contact information of 23 patients (43.9\%) was no longer correct or the patients could not be contacted by phone or mail. Two patients $(3.8 \%)$ did not want to participate in the study due to the long journey but expressed satisfaction with the results. These patients were not included in the study. The study was approved by the ethics committee of the regional State Chamber of Medicine (approval number FF 61/2016) and written informed consent was obtained from the participants indicating their permission to scientific publication of the data obtained from their treatment.

\section{Surgical technique}

Contraindications for fingertip reconstruction with a neurovascular island flap were prior injury of the digital vessels and any history of angiopathy affecting the vessels of the hand such as vasospasm, vasculitis or embolism. The flap was only raised if the proximal vessels were well perfused and not in the zone of injury. A digital Allen test or an examination with a handheld Doppler to ensure patency of both digital arteries were carried out before the procedure. Only patients compliant to postoperative rehabilitation were considered for surgery. Smoking and advanced age were not considered contraindications. Allen type I and II injuries were treated conservatively.

Antibiotic prophylaxis (second-generation cephalosporin) was administered intravenously prior to surgery. The operation was performed under regional or general anaesthesia. A pneumatic tourniquet with a pressure of $300 \mathrm{mmHg}$ and magnifying loupes were used.

After debridement of devitalized tissues, the flap was designed using a mid-lateral and a palmar oblique incision. The neurovascular bundle was identified proximal to the flap and the flap was raised above the tendon sheath (Fig. 1a, b). The neurovascular bundle was dissected until the flap did fit into the defect without any tension. Venous and arterial congestion of the neurovascular bundle has to be avoided when fitting the flap into the final position, and temporary artificial skin was used when primary closure could not be achieved at the time of the initial surgery due to swelling. If necessary, the flap was pinned with a 0.7 $\mathrm{mm} \mathrm{K}$-wire to the medullary canal of the distal phalanx to prevent proximal retraction. Z-plasties or step advancements were not used. Reconstruction with an ulnar neurovascular pedicle flap was preferred for the index finger tip and a radial-based flap for the little finger.

Patients were encouraged to perform motion exercises according to the instructions of the Hand Surgeon who performed the procedure and a physiotherapist. To ensure tension free healing of the flap emphasis was laid on motion exercises for the PIP joint with a flexed metacarpophalangeal joint. There was no standard splinting regime applied after surgery.

\section{Follow-up}

At follow-up, a patient questionnaire was completed assessing patient characteristics, occupation and satisfaction. Grip strength was measured three times for each hand using a Jamar Hand Dynamometer (Sammons Preston, Rolyan, Bolingbrook, IL, USA) on handle position two with seated participant in a neutral wrist position and the elbow by their side flexed to a right angle. The average grip strength was calculated for the left and right hand. Active range of motion for the injured fingers compared to the contralateral digits was assessed using a hand-held finger goniometer for the distal interphalangeal (DIP), proximal interphalangeal (PIP), and metacarpophalangeal (MP) joint and a standard goniometer for wrist joints. To test the patients' static two-point discrimination test (2PD), the standard protocol of the American Society for Surgery of the Hand and the International Federation of Societies for Surgery of the Hand [12] was used. Semmes-Weinstein monofilament (SWM) threshold was measured with a five-piece hand kit. The results of the SWM test were then converted to a point value that is based on the Rosen and Lundborg Physical Impairment Summary Scale [13, 14] with a maximum point value of 5 and a minimum of 0 . The tinel's sign on the finger was assessed. Scars were assessed using the Vancouver scar scale, which consists of four variables: vascularity, pigmentation, pliability and height [15]. The pain at follow-up was recorded by a numeric rating scale (0-10). Activities of daily living were measured with the Disabilities of the Arm, Shoulder and Hand (DASH) questionnaire [16]. Hook nail deformity was characterised using the Lim classification [17] (Fig. 2). The patient charts were analysed retrospectively.

The follow-up assessments were completed by an independent examiner (N. B.) who was not involved in the patient's treatment. 


\section{Fig. 1 a, b The flap is raised above the tendon sheath and the neurovascular bundle is dis- sected until the flap fits into the defect without tension}

Fig. 2 Lim classification fo hook nail deformity. The

fingertip is divided into quarters from dorsal to palmar in a lateral view. Grade 0: normal

nail appearance with no beak deformity. Grades 1-4: progressively greater downward curvature (beaking) of the nail
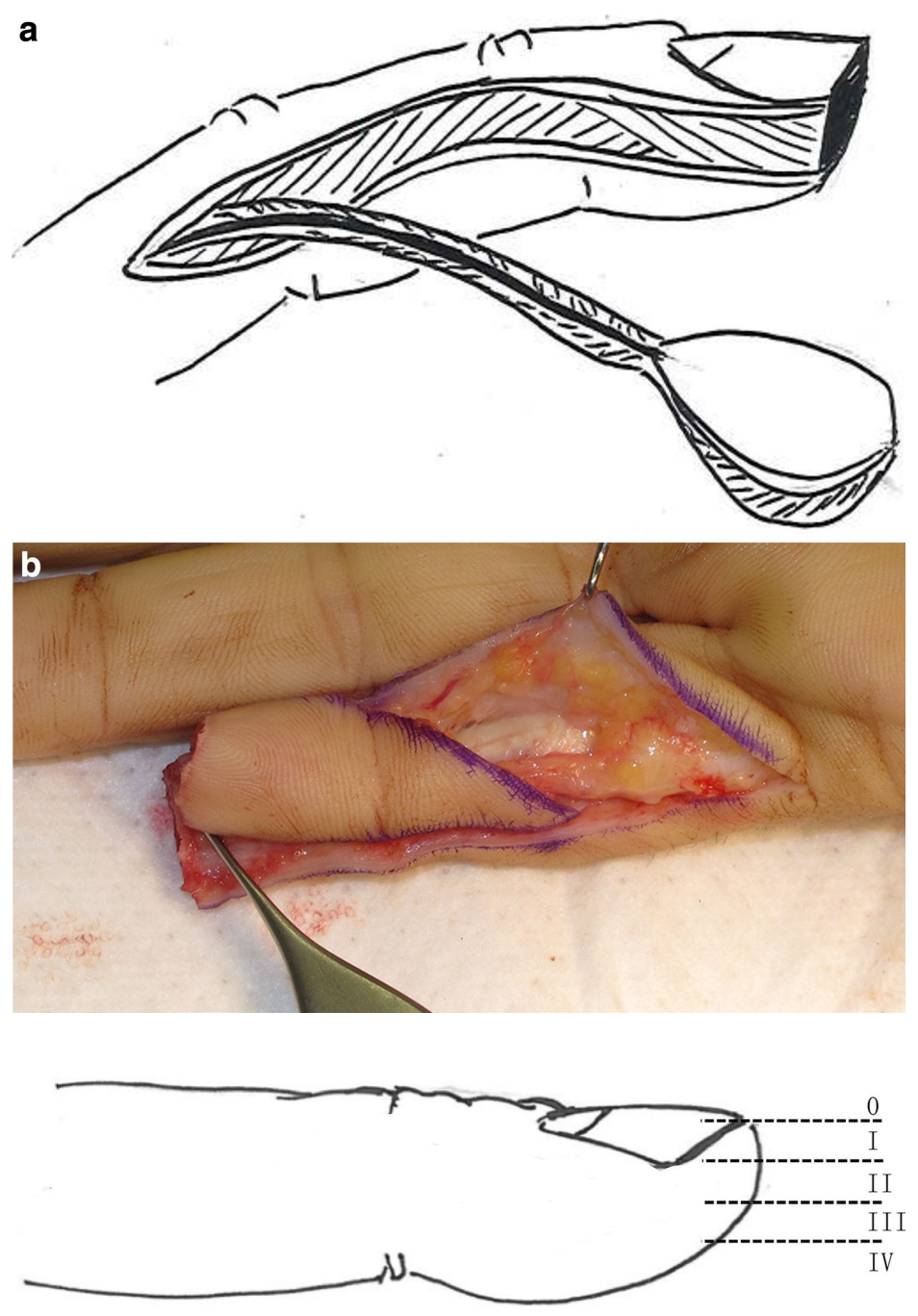

\section{Statistical methods}

Clinical variables were tabulated according to their distribution with means, range and standard deviation or expressed as percentages. After testing for normal distribution, differences between injured and contralateral side were tested using unadjusted paired $t$ tests to determine significance at the $\alpha=0.05$ level in normally distributed variables. The Mann-Whitney test was used for asymmetric distributions. 


\section{Results}

Follow-up was available for 28 patients and 29 fingers. Mean follow-up after the operation was $105 \pm 42$ months (range 42-169). The mean age of the patients at time of surgery was $42.9 \pm 15.0$ years (range $11-73$ ). The participants' characteristics are summarized in Table 1.

\section{Treatment and complications}

26 patients were operated under brachial plexus block and 2 patients under general anaesthesia. No intraoperative complications occurred. The mean in-hospital stay of all patients was $5.0 \pm 3.4$ days (range $1-12$ )

All flaps survived, yet one flap showed partial necrosis, was debrided and closed primarily 22 days after the initial operation. One reoperation was necessary due to bleeding on the fourth postoperative day in a patient with continued perioperative acetylsalicylic acid treatment. In two patients the artificial skin covering the donor side was removed after

Table 1 Demographics

\begin{tabular}{ll}
\hline Patients available for follow-up & 28 (29 fingers) \\
Gender & 24 male, 4 female \\
Affected hand & 25 right, 3 left \\
Affection of the dominant hand & 14 of $28(50 \%)$ \\
Professional activity as manual worker & 17 of $28(60.7 \%)$ \\
Affected finger & \\
D1 & 1 of $29(3.4 \%)$ \\
D2 & 10 of $29(34.5 \%)$ \\
D3 & 10 of $29(34.5 \%)$ \\
D4 & 6 of $29(20.7 \%)$ \\
D5 & 2 of $29(6.9 \%)$ \\
Allen's classification & \\
Type I & 0 \\
Type II & 0 \\
Type III & $22(75.9 \%)$ \\
Type IV & $7(24.1 \%)$ \\
Smoker & 9 of $28(32.1 \%)$ \\
\hline
\end{tabular}

swelling had subsided and the wound was either closed primarily or with a skin graft. 3 months after the initial operation, a scar correction was carried out in one patient due to painful scarring of the donor side after failure of conservative treatment. There were no infections during the postoperative course.

Complete healing was achieved after $14.6 \pm 9.6$ weeks (range 4-48). 16 of 28 patients $(57.1 \%$ ) were treated with physiotherapy (mean $15.3 \pm 14.6$ sessions, range 2-60) due to scar formation or impaired movement.

\section{Follow-up}

\section{Motion and grip strength}

Total active motion of the injured finger was $260^{\circ} \pm 23^{\circ}$ (unaffected side $268^{\circ} \pm 15^{\circ}, p=0.021$ ). The motion in the DIP, PIP and MP joint was over $95 \%$ of the contralateral side. Three patients developed an extension lag of $5^{\circ}, 15^{\circ}$ and $20^{\circ}$ in the PIP joint (Table 2) and one patient developed an extension lag of $10^{\circ}$ in the DIP joint. No patient demonstrated an extension lag in the MP joint. There was no impairment of wrist motion. The grip strength of the affected hand and of each of the affected fingers was over $70 \%$ of the contralateral side. Detailed results of hand function are summarized in Table 3 .

\section{Patient satisfaction}

On a numeric rating scale, pain at rest was mean 1.2 and motion pain was mean 2.4. 21 participants (75\%) reported no pain at rest and 15 of $28(53.6 \%)$ no motion pain. 14 of $28(50 \%)$ of participants reported an increased sensitivity to cold temperatures and 5 of 28 (17.9\%) tenderness of the reconstructed fingertip. The overall DASH score was 16.0. The score of the DASH work module was $17.2(n=20)$ and the score of the sport module was $15.5(n=19)$. Patien satisfaction was $8.7 \pm 1.4$ on an eleven point rating scale $(0=$ extremely dissatisfied and 10 extremely satisfied $)$. All participants would have had the same surgical procedure again (as opposed to a qualified amputation) knowing the final outcome.
Table 2 Demographics and clinical results of patients with a decrease in the proximal interphalangeal (PIP) joint extension angle

\begin{tabular}{llllllll}
\hline Patient & $\begin{array}{l}\text { PIP joint } \\
\text { extension } \\
\text { lag }\end{array}$ & Age in years & Injured finger & $\begin{array}{l}\text { Follow-up } \\
\text { in months }\end{array}$ & $\begin{array}{l}\text { Time to } \\
\text { heal in } \\
\text { weeks }\end{array}$ & $\begin{array}{l}\text { Sessions } \\
\text { of physio- } \\
\text { therapy }\end{array}$ & DASH score \\
\hline 1 & $5^{\circ}$ & 64 & II & 45 & 6 & 18 & 5.8 \\
2 & $15^{\circ}$ & 34 & IV & 44 & 10 & 24 & 6.7 \\
3 & $20^{\circ}$ & 45 & II & 169 & 12 & 10 & 29.2 \\
\hline
\end{tabular}

$D A S H$ disabilities of the arm, shoulder and hand questionnaire ${ }^{\mathrm{a}}$ At the time of injury 


\begin{tabular}{lcc} 
Table 3 Hand function & \\
\hline & $\begin{array}{l}\text { Average (SD) } \\
\text { at follow-up }\end{array}$ & $\begin{array}{l}\text { Comparison to } \\
\text { contralateral side } \\
(\%)\end{array}$ \\
\hline Total active motion in degrees & $260(23)$ & 97 \\
DIP motion in degrees & $81(19)$ & 96 \\
PIP motion in degrees & $96(6)$ & 98 \\
MP motion in degrees & $87(9)$ & 99 \\
Grip strength at wrist in kg & $33(13)$ & 91 \\
Grip strength at injured finger in & & \\
kg for & & \\
DII & $5(3)$ & 80 \\
DIII & $4(2)$ & 100 \\
DIV & $5(2)$ & 79 \\
DV & $1(1)$ & 73 \\
\hline
\end{tabular}

$S D$ standard deviation

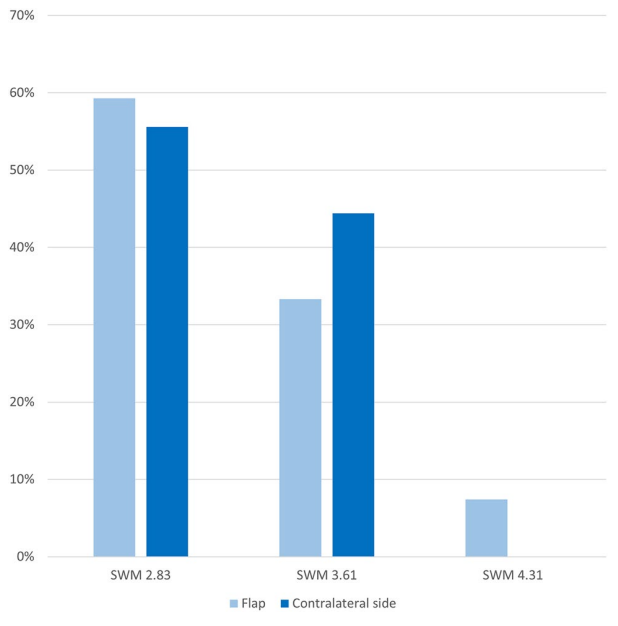

Fig. 3 Distribution of Semmes-Weinstein monofilament (SWM) results of the injured finger (flap) compared with the contralateral finger. There was no statistical significant difference in the Mann-Whitney test between the injured and uninjured side $(p=0.976)$

\section{Neurological assessment}

The Semmes-Weinstein monofilament results are shown in Fig. 3. 9 patients were able to feel the 3.61 (diminished light touch) at the reconstructed fingertip and 2 patients feeling 4.31 (diminished protective sensation). The remaining patients were able to feel 2.83 (normal). In 23 patients the results of the injured finger were equal or better compared to the uninjured side. There was no statistical significant difference in the Mann-Whitney test between the injured and uninjured side $(p=0.976)$.

Static two-point discrimination was $5.1 \pm 1.7 \mathrm{~mm}$ (range $2-8$ ), contralateral side $3.6 \pm 1.0 \mathrm{~mm}$ (range $2-6, p=0.005$ ). In nine patients the results of the injured finger were equal or better compared to the uninjured side.

Four patients reported a tingling sensation over the course of the nerve and seven patients (including the latter 4) showed a mild Tinel's sign after careful examination despite conservative treatment. None of the seven patients described this sensation as disturbing.

\section{Appearance}

Mean flap size at follow-up was $4.7 \pm 0.6 \mathrm{~cm}^{2}$. The scars were rated 0.6 (range $0-3$ ) on the Vancouver scar scale by the independent examiner.

The nail plate could be preserved in 22 of 29 (75.9\%) fingers. In 10 of the reconstructed fingertips with preserved nail, the nails were shorter than the contralateral side (mean $1.8 \pm 0.6 \mathrm{~mm}$ shorter, range $1-3 \mathrm{~mm}$ ). 14 fingers showed hook nail deformity at follow-up. According to the Lim classification 1 of 14 nails (7.1\%) showed grade 3 breaking of the nail and 7 of $14(50.0 \%)$ showed grade 2 . The remaining 6 nails (42.9\%) were classified as grade 1 . All patients with preserved nail were satisfied, that the finger had a nail. No patient reported further treatment due to hook nail development, infections or other complications caused by the nail deformity.

\section{Occupation}

27 of 28 patients were working at the time of injury, one participant had retired. The average absence from work was $6.1 \pm 3.3$ weeks (range $1-12$ weeks). In the 18 of 27 patients that received $\mathrm{WC}$, the average sickness leave was $6.9 \pm 3.4$ weeks (non-WC patients $4.4 \pm 2.5, p<0.05$ ). All 27 patients, including the 17 blue collar workers, returned to their original occupation. All patients returned to the same work schedule, that they had before the injury.

\section{Discussion}

Choosing a suitable treatment for an individual patient with fingertip injuries remains challenging despite many options. There is an ongoing discussion among hand surgeons which treatment is best for different types of fingertip injuries [18-20]. The therapeutic armamentarium consists of a variety of conservative and surgical treatments including revision amputation [2, 20-22]. Publications are very heterogeneous regarding mechanism and level of injury, demographic characteristics and treatment technique and

\section{Publikation}

$-132-$ 
thus results are sometimes hard to compare. Many studies are published describing new surgical techniques for fingertip reconstruction. However, most studies only report short- or mid-term outcomes. The homodigital island flap meets a lot of the criteria for an ideal reconstruction, yet only few studies are published with a longer follow-up. Knowing the long-term outcome would help surgeons and patients to make an informed decision when considering the right option for the individual patient. In this study, we showed that a functional digit can be expected in most patients after homodigital island flap reconstruction of the fingertip at follow-up of more than 8 years.

One main goal of the treatment of fingertip injuries is to restore a near normal active motion without flexion contracture. The present study shows total finger joint movement over $95 \%$ of the contralateral side, which is rated "good" according to Kleinert [23]. 14.3\% developed an extension lag of the PIP or DIP joints with a maximum of $20^{\circ}$. In a study of 63 fingers [10] 23.8\% showed an extension lag in the interphalangeal joints at a mean follow-up at 46 months. In this study, extension exercises and splinting were used, when a flexion contracture persisted, but no further information is given on this topic. In another study [7], 9.5\% of the 115 fingers showed impaired motion at a mean followup at 41 months. The maximum extension lag in the PIP joint was $25^{\circ}$. Plaster splint immobilization was applied for only 1 week after the operation and an extension lag splint was prescribed in case of an early extension lag. Usami [9] followed 17 patients at mean 12.1 months and found mild PIP joint contractures in $23.5 \%$ of fingers. We attribute the overall good outcome in motion in our series mainly to the fact that our study had a longer follow-up than other studies with small improvements of the extension lag over time.

A multivariate analysis of 44 fingers with average 33 months follow-up detected age over 59 years as an independent predictor of flexion contracture of the PIP joint [24]. However, we could not confirm this finding, as in our series only one of the three patients with PIP joint flexion contracture was over 59 years while the other ones were noticeably younger.

Mandatory splinting after surgery remains controversial. As our results are not inferior to the results of other authors that splint every finger, mandatory splinting might not be necessary. Other reconstructive options such as cross-finger flaps or palmar flaps require additional immobilization and bear the risk of PIP flexion contracture, too [3]. The reverse flow island flap with neurorrhaphy can minimize this risk, yet discriminatory sensation seems to be better in the direct flow flap [8].

Reaching the optimal sensation of the reconstructed fingertip is another main goal of treatment [25]. In a study with 115 fingers $87.3 \%$ of fingers showed a sensitivity of 4.31 or better in the SWM test after mean follow-up of 41 months
[7]. However, there was a wide variety of $2 \mathrm{PD}$ ranging from 2 to $11 \mathrm{~mm}$. $66.4 \%$ fingers had a 2PD of 2-4 mm.

In the study published by Varitimidis and colleagues [10] static 2PD was mean $4 \mathrm{~mm}$ with $93.7 \%$ of fingers having a static two-point discrimination between 3 and $6 \mathrm{~mm}$. In our study, static two-point discrimination was mean $5.1 \mathrm{~mm}$, which is rated as "excellent" according to the Modified American Society for Surgery of the Hand guidelines to stratify 2PD [26]. All patients (100\%) showed a sensitivity of 4.31 or better in the SWM results. Our results of static two-point discrimination are comparable to other studies, while the results for SWM seem to be slightly better in our long-term study compared with studies that have less follow-up.

There are only few studies addressing the nail after fingertip reconstruction with a neurovascular island flap, but most authors mention hook nail deformity as possible complication [10]. In a series of 115 fingers hook nails were detected in 8 fingers (7\%) at mean 41 months. Three patients underwent flap reconstruction for hook nail deformity and were satisfied with their result [7]. The findings of Lim [17] suggests, that nail breaking might worsen over time in fingertips that were reconstructed with a spiral flap. In our series nearly half of the participants demonstrated varying degrees of hook nail deformity at long-term follow-up, yet no patient complained about it at follow-up or needed treatment. This promotes that patients should be counselled about the possibility of nail deformity before surgery.

There is an ongoing discussion regarding the best treatment for fingertip amputations. Despite the high frequency of this injury no evidence-based standard has been established so far.

One main alternative to flap reconstruction is conservative management [11] e.g. with semi-occlusive dressings [27]. Several studies reported good results with near-normal sensibility and without donor side morbidity not only in distal fingertip amputations but also in patients with Allen's type III/IV injuries [18, 28-30]. The few existing comparative studies indicate, that surgical reconstruction of the fingertip might offer little to no advantage over conservative treatment [31,32]. Accordingly, our own treatment standard has shifted from flap surgery for most patients with exposed bone until 2014 to a more conservative approach based on the characteristics and mechanism of the amputation and the individual needs of the patient.

There are some limitations to this study. First, it is a single-centre cohort study. Second, follow-up was only available for 28 patients, and thus this is a small sample size Third, as the Allen classification [11] was used to classify injuries there is no information on the direction of injury or the shape of the defect. Using more complex classifications with more subdivisions to describe the injury would be more exact [33], but these were considered too complicated by 
some authors [10] and are thus not commonly used in other studies $[9,8,7]$.

\section{Conclusion}

A very high patient satisfaction and good long-term function can be achieved with the anterograde homodigital neurovascular island flap in a one-step operation with immediate sensitivity of the reconstructed fingertip without compromising an uninjured adjacent finger. Mandatory splinting or z-plasties to avoid disabling PIP joint flexion contracture might not be necessary. A functional digit with near normal length and good movement can be expected in most of the patients. Further studies are needed to compare the longterm clinical outcome of different therapeutic options.

Acknowledgements We thank our librarian Claudia Diemann-Paeth for helping with the literature research.

Funding The authors received no financial support for the research, authorship, and/or publication of this article.

\section{Compliance with ethical standards}

Conflict of interest The authors declare that they have no conflict of interest.

Ethical approval The study was approved by the ethics committee of the regional State Chamber of Medicine (approval number FF 61/2016) and written informed consent was obtained from the participants indicating their permission to scientific publication of the data.

\section{References}

1. Sorock GS, Lombardi DA, Hauser RB, Eisen EA, Herrick RF, Mittleman MA (2002) Acute traumatic occupational hand injuries: type, location, and severity. J Occup Environ Med 44(4):345-351

2. Harenberg PS, Jakubietz RG, Jakubietz MG, Schmidt K, Meffert RH (2012) Reconstruction of the thumb tip using palmar neurovascular flaps. Operative Orthopadie und Traumatologie 24(2):116-121. https://doi.org/10.1007/s00064-011-0081-3

3. Katz RD (2013) The anterograde homodigital neurovascular island flap. J Hand Surg Am 38(6):1226-1233. https://doi. org/10.1016/j.jhsa.2013.03.025

4. Borman H, Maral T, Tancer M (2000) Fingertip reconstruction using two variations of direct-flow homodigital neurovascular island flaps. Ann Plast Surg 45(1):24-30

5. Evans DM, Martin DL (1988) Step-advancement island flap for fingertip reconstruction. Br J Plast Surg 41(2):105-111

6. Lanzetta M, Mastropasqua B, Chollet A, Brisebois N (1995) Versatility of the homodigital triangular neurovascular island flap in fingertip reconstruction. J Hand Surg (Edinburgh, Scotland) 20(6):824-829

7. Kayalar M, Bal E, Toros T, Sugun ST, Ozaksar K, Gurbuz Y (2011) The outcome of direct-flow neurovascular island flaps in pulp defects. Acta orthopaedica et traumatologica turcica 45(3):175-184. https://doi.org/10.3944/aott.2011.2429
8. Braga-Silva J, Gehlen D, Bervian F, da Cunha GL, Padoin AV (2009) Randomized prospective study comparing reverse and direct flow island flaps in digital pulp reconstruction of the fingers. Plast Reconstr Surg 124(6):2012-2018. https://doi. org/10.1097/PRS.0b013e3181bcf2b9

9. Usami S, Kawahara S, Yamaguchi Y, Hirase T (2015) Homodigital artery flap reconstruction for fingertip amputation: a comparative study of the oblique triangular neurovascular advance ment flap and the reverse digital artery island flap. J Hand Surg Eur 40(3):291-297. https://doi.org/10.1177/175319341351513

10. Varitimidis SE, Dailiana ZH, Zibis AH, Hantes M, Bargiota K, Malizos KN (2005) Restoration of function and sensitivity utilizing a homodigital neurovascular island flap after amputation injuries of the fingertip. J Hand Surg (Edinburgh, Scotland) 30(4):338-342. https://doi.org/10.1016/j.jhsb.2005.04.014

11. Allen MJ (1980) Conservative management of finger tip injuries in adults. Hand 12(3):257-265

12. Dellon AL (1978) The moving two-point discrimination test: clinical evaluation of the quickly adapting fiber/receptor system. J Hand Surg Am 3(5):474-481

13. MacDermid JC (2005) Measurement of health outcomes following tendon and nerve repair. J Hand Ther Off J Am Soc Hand Ther 18(2):297-312. https://doi.org/10.1197/j.jht.2005.02.009

14. Rosen B, Dahlin LB, Lundborg G (2000) Assessment of functional outcome after nerve repair in a longitudinal cohort. Scand J Plast Reconstr Surg Hand Surg 34(1):71-78

15. Sullivan T, Smith J, Kermode J, McIver E, Courtemanche DJ (1990) Rating the burn scar. J Burn Care Rehabilit 11(3):256-260

16. Hudak PL, Amadio PC, Bombardier C (1996) Development of an upper extremity outcome measure: the DASH (disabilities of the arm, shoulder and hand) [corrected]. The Upper Extremity Collaborative Group (UECG). Am J Ind Med 29(6):602-608. https:// doi.org/10.1002/(sici)1097-0274(199606)29:6\%3c602:aid-ajim4 $\% 3$ e3.0.co;2-1

17. Lim GJ, Yam AK, Lee JY, Lam-Chuan T (2008) The spira flap for fingertip resurfacing: short-term and long-term results. J Hand Surg Am 33(3):340-347. https://doi.org/10.1016/j. jhsa.2007.12.005

18. Krauss EM, Lalonde DH (2014) Secondary healing of fingertip amputations: a review. Hand (New York, NY) 9(3):282-288. http ://doi.org/10.1007/s11552-014-9663-5

19. Champagne L, Hustedt JW, Walker R, Wiebelhaus J, Nystrom NA (2016) Digital tip amputations from the perspective of the nail Adv Orthop 2016:1967192. https://doi.org/10.1155/2016/1967 92

20. Unglaub F, Langer MF, Unglaub JM, Müller LP, Hahn P, Spies CK, Löw S (2018) Defect coverage of fingers and thumb: Indications and treatment. Unfallchirurg 121(4):321-334. https://doi org/10.1007/s00113-018-0469-8

21. Tan HL, Tan DY, Zhao JK (2013) Treatment of thumb soft-tissue defects using a bipedicle island flap of the index finger: anatomical basis and clinical application. Arch Orthop Trauma Surg 133(5):721-728. https://doi.org/10.1007/s00402-013-1704-y

22. Zhou X, Wang L, Mi J, Xu Y, Rui Y, Xue M, Shen X, Qiang L (2015) Thumb fingertip reconstruction with palmar V-Y flaps combined with bone and nail bed grafts following amputation. Arch Orthop Trauma Surg 135(4):589-594. https://doi org/10.1007/s00402-015-2163-4

23. Kleinert HE, Verdan C (1983) Report of the Committee on Tendon Injuries (International Federation of Societies for Surgery of the Hand). J Hand Surg Am 8(5 Pt 2):794-798

24. Nakanishi A, Omokawa S, Iida A, Kaji D, Tanaka Y (2015) Predictors of proximal interphalangeal joint flexion contracture after homodigital island flap. J Hand Surg Am 40(11):2155-2159. http ://doi.org/10.1016/j.jhsa.2015.08.008 
25. Sano K, Ozeki S, Kimura K, Hyakusoku H (2008) Relationship between sensory recovery and advancement distance of oblique triangular flap for fingertip reconstruction. J Hand Surg Am 33(7):1088-1092. https://doi.org/10.1016/j.jhsa.2008.02.022

26. Mermans JF, Franssen BB, Serroyen J, Van der Hulst RR (2012) Digital nerve injuries: a review of predictors of sensory recovery after microsurgical digital nerve repair. Hand (New York, NY) 7(3):233-241. https://doi.org/10.1007/s11552-012-9433-1

27. Mennen U, Wiese A (1993) Fingertip injuries management with semi-occlusive dressing. J Hand Surg (Edinburgh, Scotland) 18(4):416-422

28. Peterson SL, Peterson EL, Wheatley MJ (2014) Management of fingertip amputations. J Hand Surg Am 39(10):2093-2101. https ://doi.org/10.1016/j.jhsa.2014.04.025

29. Quadlbauer S, Pezzei C, Jurkowitsch J, Beer T, Keuchel T, Hausner T, Leixnering M (2017) The semi-occlusive dressing in treating Allen III and IV fingertip injuries as an alternative to local skin flaps. Unfallchirurg 120(11):961-968. https://doi. org/10.1007/s00113-016-0237-6

30. Yuan F, McGlinn EP, Giladi AM, Chung KC (2015) A systematic review of outcomes after revision amputation for treatment of traumatic finger amputation. Plast Reconstr Surg 136(1):99-113. https://doi.org/10.1097/prs.0000000000001487

31. Weichman KE, Wilson SC, Samra F, Reavey P, Sharma S, Haddock NT (2013) Treatment and outcomes of fingertip injuries at a large metropolitan public hospital. Plast Reconstr Surg 131(1): 107-112. https://doi.org/10.1097/PRS.0b013e3182729ec2

32. van den Berg WB, Vergeer RA, van der Sluis CK, Ten Duis HJ, Werker PM (2012) Comparison of three types of treatment modal ities on the outcome of fingertip injuries. J Trauma Acute Care Surg 72(6):1681-1687. https://doi.org/10.1097/TA.0b013e3182 $48 \mathrm{bc} 8 \mathrm{c}$

33. Smith KL, Elliot D (2000) The extended Segmuller flap. Plast Reconstr Surg 105(4):1334-1346

Publisher's Note Springer Nature remains neutral with regard to jurisdictional claims in published maps and institutional affiliations. 


\section{Danksagung}

Herrn Professor Dr. med. Michael Sauerbier, Chefarzt der Abteilung für Plastische, Handund Rekonstruktive Chirurgie der Berufsgenossenschaftlichen Unfallklinik Frankfurt am Main, danke ich an erster Stelle für die Möglichkeit der Promotion in seiner Abteilung.

Mein größter Dank gilt Frau Dr. med. Annika Arsalan-Werner, Oberärztin der Abteilung für Plastische, Hand- und Rekonstruktive Chirurgie, die mich bei meiner Arbeit stets mit viel Verständnis und jederzeit mit ihrem fachlichen Wissen und ihrer langjährigen Erfahrung unterstützte.

Ihre Förderung hat es mir ermöglicht, meine wissenschaftlichen Kenntnisse aus dem Studium auszubauen, was mein Interesse an der Forschung geweckt hat.

Danksagung - - $136-$ 


\section{Schriftliche Erklärung}

Ich erkläre ehrenwörtlich, dass ich die dem Fachbereich Medizin der Johann Wolfgang Goethe-Universität Frankfurt am Main zur Promotionsprüfung eingereichte Dissertation mit dem Titel:

\section{Langzeitergebnisse nach plastisch-chirurgischer Defektdeckung durch neurovaskulären Insel-Lappen bei Fingerkuppenverletzungen}

in der Abteilung für Plastische-, Hand- und Rekonstruktive Chirurgie der Berufsgenossenschaftlichen Unfallklinik Frankfurt am Main unter Betreuung und Anleitung von Prof. Dr. Dr. med. M. Sauerbier mit Unterstützung durch Dr. med. Annika ArsalanWerner ohne sonstige Hilfe selbst durchgeführt und bei der Abfassung der Arbeit keine anderen als die in der Dissertation angeführten Hilfsmittel benutzt habe. Darüber hinaus versichere ich, nicht die Hilfe einer kommerziellen Promotionsvermittlung in Anspruch genommen zu haben.

Ich habe bisher an keiner in- oder ausländischen Universität ein Gesuch um Zulassung zur Promotion eingereicht. Die vorliegende Arbeit wurde bisher nicht als Dissertation eingereicht.

Vorliegende Ergebnisse der Arbeit wurden in folgendem Publikationsorgan veröffentlicht: Arsalan-Werner A, Brui N, Mehling I, Schlageter M, Sauerbier M. Long-term outcome of fingertip reconstruction with the homodigital neurovascular island flap. Arch Orthop Trauma Surg. 2019;139(8):1171-1178.

Frankfurt am Main, den 15. September 2020

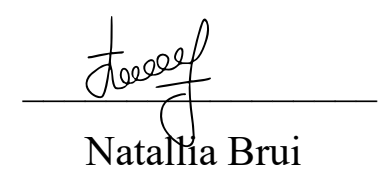

